\title{
Reduction by symmetries in singular quantum-mechanical problems: general scheme and application to Aharonov-Bohm model
}

\author{
A.G. Smirnova) \\ I. E. Tamm Theory Department, P. N. Lebedev Physical Institute, Leninsky prospect 53, \\ Moscow 119991, Russia
}

\begin{abstract}
We develop a general technique for finding self-adjoint extensions of a symmetric operator that respect a given set of its symmetries. Problems of this type naturally arise when considering twoand three-dimensional Schrödinger operators with singular potentials. The approach is based on constructing a unitary transformation diagonalizing the symmetries and reducing the initial operator to the direct integral of a suitable family of partial operators. We prove that symmetry preserving self-adjoint extensions of the initial operator are in a one-to-one correspondence with measurable families of self-adjoint extensions of partial operators obtained by reduction. The general scheme is applied to the three-dimensional Aharonov-Bohm Hamiltonian describing the electron in the magnetic field of an infinitely thin solenoid. We construct all self-adjoint extensions of this Hamiltonian, invariant under translations along the solenoid and rotations around it, and explicitly find their eigenfunction expansions.
\end{abstract}

\section{INTRODUCTION}

The spectral analysis of Schrödinger operators can be often facilitated if some symmetries of the problem are known. This happens if the spectral problem for the operators representing symmetries can be explicitly solved. In this case, the initial operator can be represented as a direct sum (or, more generally, direct integral) of partial operators corresponding to fixed eigenvalues of symmetries. In this way, twoand three-dimensional problems can be often reduced to solving certain one-dimensional Schrödinger equations (this is the case, for example, for spherically symmetric problems).

The present paper is concerned with such a reduction by symmetries in the case of singular quantummechanical problems. It is well known ${ }^{1,2}$ that strong singularities in the potential may lead to the lack of self-adjointness of the corresponding Schrödinger operator on its natural domain. As a result, the quantum model is no longer fixed uniquely by the potential and different quantum dynamics described by various self-adjoint extensions of the initial Schrödinger operator are possible. In this paper, we are interested in the extensions that respect some given set of symmetries of the initial operator. We propose a general technique for the reduction of such symmetry preserving extensions and apply it to the spectral analysis of the three-dimensional Aharonov-Bohm Hamiltonian describing the electron in the magnetic field of an infinitely thin solenoid.

In a general form, the problem can be posed as follows. Suppose $H$ is a closed operator in a separable Hilbert space $\mathfrak{H}$ and $\mathfrak{X}$ is a subset of the algebra $L(\mathfrak{H})$ of linear bounded everywhere defined operators in $\mathfrak{H}$. We assume that $\mathfrak{X}$ is involutive (i.e., the adjoint $T^{*}$ of every $T \in \mathfrak{X}$ belongs to $\mathfrak{X}$ ) and consists of pairwise commuting operators. Further, we assume $\mathfrak{X}$ is a set of symmetries of $H$, by which we mean that $H$ commutes with elements of $\mathfrak{X}$ in the sense of the next definition.

Definition I.1. A linear operator $H$ in $\mathfrak{H}$ with the domain $D_{H}$ is said to commute with $T \in L(\mathfrak{H})$ if $T \Psi \in D_{H}$ and $H T \Psi=T H \Psi$ for any $\Psi \in D_{H}$.

Our aim is to show how spectral decompositions of symmetries can be used to represent closed (and, in particular, self-adjoint) symmetry preserving extensions of $H$ as direct integrals of suitable partial operators. To clarify main ideas, we assume for a while that $\mathfrak{H}$ falls into an orthogonal direct sum of eigenspaces of symmetries. That is, we suppose that there is a countable family $\{\mathfrak{S}(s)\}_{s \in \mathscr{S}}$ of nontrivial closed pairwise orthogonal subspaces of $\mathfrak{H}$ such that

$$
\mathfrak{H}=\bigoplus_{s \in \mathscr{S}} \mathfrak{S}(s)
$$

and

$$
T \Psi=g_{T}(s) \Psi, \quad \Psi \in \mathfrak{S}(s),
$$

\footnotetext{
a) Electronic mail: smirnov@lpi.ru
} 
for every $s \in \mathscr{S}$ and $T \in \mathfrak{X}$, where $g_{T}(s)$ are some complex numbers (since $\mathfrak{S}(s)$ are nontrivial, the eigenvalues $g_{T}(s)$ are defined uniquely). Let $P_{s}$ denote the orthogonal projection of $\mathfrak{H}$ onto $\mathfrak{S}(s)$. We would like to have a decomposition of the form

$$
H=\bigoplus_{s \in \mathscr{S}} \mathcal{H}(s)
$$

where $\mathcal{H}(s)$ are closed operators in $\mathfrak{S}(s)$ for all $s \in \mathscr{S}$ and $\bigoplus_{s \in \mathscr{S}} \mathcal{H}(s)$ is, by definition, the operator in $\mathfrak{H}$ whose graph consists of all $(\Psi, \tilde{\Psi}) \in \mathfrak{H} \oplus \mathfrak{H}$ such that $\left(P_{s} \Psi, P_{s} \tilde{\Psi}\right)$ is in the graph of $\mathcal{H}(s)$ for every $s \in \mathscr{S}$. As the operator $\bigoplus_{s \in \mathscr{S}} \mathcal{H}(s)$ obviously commutes with all projections $P_{s}$, equality (2) can hold only if $H$ commutes with $P_{s}$ for every $s \in \mathscr{S}$. In fact, the latter condition is also sufficient for the existence of decomposition (2) (see Theorems 2 and 3 in Sec. 45 of Ref. $\underline{3}$ ). Given a decomposition of form (2), it can be easily shown that $\mathcal{H}(s)$ is actually the restriction of $H$ to the domain $D_{H} \cap \mathfrak{S}(s)$ for every $s \in \mathscr{S}$. Thus, the partial operators $\mathcal{H}(s)$ are uniquely determined by $H$.

In general, the condition that $\mathfrak{S}(s)$ are eigenspaces of symmetries is insufficient to guarantee that $H$ commutes with $P_{s}$ for all $s \in \mathscr{S}$ (otherwise we could choose $\mathfrak{X}$ to be the one-element set containing the identity operator in $\mathfrak{H}$ and consider the decomposition $\mathfrak{H}=\mathfrak{H}^{\prime} \oplus \mathfrak{H}^{\prime}$, where $\mathfrak{H}^{\prime}$ is an arbitrary closed subspace of $\mathfrak{H}$; it would follow that every closed operator in $\mathfrak{H}$ commutes with the orthogonal projection onto $\mathfrak{H}^{\prime}$ ). The commutation between $H$ and $P_{s}$ can be ensured, however, if we require that

$$
P_{s} \in \mathcal{A}(\mathfrak{X})
$$

for every $s \in \mathscr{S}$, where $\mathcal{A}(\mathfrak{X})$ is the smallest strongly closed subalgebra of $L(\mathfrak{H})$ containing the set $\mathfrak{X}$ and the identity operator in $\mathfrak{H}$ (in other words, $\mathcal{A}(\mathfrak{X})$ is the strong closure of the algebra generated by $\mathfrak{X}$ and the identity operator). Indeed, let $\mathcal{M}$ be the subset of $L(\mathfrak{H})$ consisting of all operators commuting with $H$. Since the sum and product of any two operators in $L(\mathfrak{H})$ commuting with $H$ also commute with $H$, we conclude that $\mathcal{M}$ is an algebra. Moreover, it easily follows from the closedness of $H$ that $\mathcal{M}$ is closed in the strong operator topology (see Lemma 1 in Ref. 4 ) and, hence, $\mathcal{A}(\mathfrak{X}) \subset \mathcal{M}$. Condition (3) therefore implies that $P_{s} \in \mathcal{M}$ or, in other words, that $H$ commutes with $P_{s}$ for all $s \in \mathscr{S}$.

We shall say that an eigenspace decomposition of form (11) is exact for $\mathfrak{X}$ if condition (3) is fulfilled for all $s \in \mathscr{S}$. Given an exact decomposition for $\mathfrak{X}$, it is easy to describe symmetry preserving closed extensions of $H$. Indeed, suppose (2) holds and a closed extension $\tilde{\mathcal{H}}(s)$ of $\mathcal{H}(s)$ is given for every $s \in \mathscr{S}$. Then the operator $\tilde{H}=\bigoplus_{s \in \mathscr{S}} \tilde{\mathcal{H}}(s)$ is clearly a closed extension of $H$ commuting with all elements of $\mathfrak{X}$. Conversely, if $\tilde{H}$ is a closed extension of $H$ commuting with all elements of $\mathfrak{X}$, then the above considerations applied to $\tilde{H}$ instead of $H$ show that $\tilde{H}$ falls into the direct sum of its partial operators $\tilde{\mathcal{H}}(s)$, which are obviously closed extensions of $\mathcal{H}(s)$. Moreover, $\tilde{H}$ is densely defined if and only if all $\tilde{\mathcal{H}}(s)$ are densely defined, in which case we have $\tilde{H}^{*}=\bigoplus_{s \in \mathscr{S}} \tilde{\mathcal{H}}(s)^{*}$. Hence, $\tilde{H}$ is self-adjoint if and only if $\tilde{\mathcal{H}}(s)$ is self-adjoint for every $s \in \mathscr{S}$.

Thus, given an exact decomposition for $\mathfrak{X}$, the operator $H$ falls into the direct sum of its partial operators $\mathcal{H}(s)$, and the symmetry preserving closed (resp., self-adjoint) extensions of $H$ are precisely the direct sums of closed (resp., self-adjoint) extensions of $\mathcal{H}(s)$.

Exactness condition (3) can be given another, equivalent, formulation that is better suited for verifying in concrete applications. More specifically, we claim that (3) holds for all $s \in \mathscr{S}$ if and only if the following condition is fulfilled:

(E) For every $s, s^{\prime} \in \mathscr{S}$ such that $s \neq s^{\prime}$, there is $T \in \mathfrak{X}$ such that $g_{T}(s) \neq g_{T}\left(s^{\prime}\right)$.

Indeed, suppose $(\mathrm{E})$ holds. Since $\mathfrak{X}$ is involutive, the elements of $\mathcal{A}(\mathfrak{X})$ can be characterized using von Neumann's bicommutant theorem (see Sec. III for details): an operator in $L(\mathfrak{H})$ belongs to $\mathcal{A}(\mathfrak{X})$ if and only if it commutes with every element of commutant of $\mathfrak{X}$, i.e., with every operator in $L(\mathfrak{H})$ that commutes with all elements of $\mathfrak{X}$. To prove $(3)$, we have to verify this condition for $P_{s}$. Let $R \in L(\mathfrak{H})$ commute with all elements of $\mathfrak{X}$. Since $T P_{s}=P_{s} T=g_{T}(s) P_{s}$ for every $s \in \mathscr{S}$ and $T \in \mathfrak{X}$, we have $P_{s^{\prime}} R T P_{s}=g_{T}(s) P_{s^{\prime}} R P_{s}=g_{T}\left(s^{\prime}\right) P_{s^{\prime}} R P_{s}$ for all $T \in \mathfrak{X}$ and $s, s^{\prime} \in \mathscr{S}$. In view of (E), this implies that $P_{s^{\prime}} R P_{s}=0$ for all $s, s^{\prime} \in \mathscr{S}$ such that $s \neq s^{\prime}$. As $\Psi=\sum_{s \in \mathscr{S}} P_{s} \Psi$ for any $\Psi \in \mathfrak{H}$, we obtain

$$
P_{s} R \Psi=\sum_{s^{\prime} \in \mathscr{S}} P_{s} R P_{s^{\prime}} \Psi=P_{s} R P_{s}=\sum_{s^{\prime} \in \mathscr{S}} P_{s^{\prime}} R P_{s} \Psi=R P_{s} \Psi
$$

for every $s \in \mathscr{S}$ and $\Psi \in \mathfrak{H}$. Thus, $P_{s}$ commutes with $R$ for every $s \in \mathscr{S}$, and (3) is proved. We now show that, conversely, (3) implies (E). Suppose, to the contrary, that (3) holds and there exist $s_{1}, s_{2} \in \mathscr{S}$ such that $s_{1} \neq s_{2}$ and $g_{T}\left(s_{1}\right)=g_{T}\left(s_{2}\right)$ for every $T \in \mathfrak{X}$. We then have $\tilde{P} T=T \tilde{P}=g_{T}\left(s_{1}\right) \tilde{P}$ for every $T \in \mathfrak{X}$, where $\tilde{P}=P_{s_{1}}+P_{s_{2}}$ is the orthogonal projection of $\mathfrak{H}$ onto $\mathfrak{S}\left(s_{1}\right) \oplus \mathfrak{S}\left(s_{2}\right)$. It follows that every element 
of $\mathfrak{X}$ commutes with $\tilde{P} R \tilde{P}$ for every $R \in L(\mathfrak{H})$. In view of $(\underline{3})$ and the characterization of elements of $\mathcal{A}(\mathfrak{X})$ given above, this implies that $P_{s_{1}}$ commutes with $\tilde{P} R \tilde{P}$ for every $R \in L(\mathfrak{H})$, which is obviously false. We thus arrive at a contradiction and our claim is proved.

The above discussion is based on the assumption that the Hilbert space $\mathfrak{H}$ falls into a direct sum of eigenspaces of symmetries. In general, this assumption does not hold and one has to use direct integral decompositions instead. More specifically, we can try to find a positive measure $\nu$, a $\nu$-measurable family $\mathfrak{S}$ of Hilbert spaces, and a unitary operator $V: \mathfrak{H} \rightarrow \int^{\oplus} \mathfrak{S}(s) d \nu(s)$ such that every $T \in \mathcal{X}$ is representable in the form $V^{-1} \mathcal{T}_{g}^{\nu, \mathfrak{S}} V$ for some $\nu$-measurable complex function $g$, where $T_{g}^{\nu, \mathfrak{S}}$ is the operator of multiplication by $g$ in $\int^{\oplus} \mathfrak{S}(s) d \nu(s)$ (we refer the reader to Appendix B for the notions related to direct integrals of Hilbert spaces). A triple $(\nu, \mathfrak{S}, V)$ satisfying this condition is said to be a diagonalization for $\mathfrak{X}$. Since countable direct sums of separable Hilbert spaces can be always identified with suitable direct integrals (see Sec. B3 and formula (B2)), the eigenspace decompositions considered above can be viewed as a particular case of diagonalizations, with the role of $\nu$ and $V$ played by the counting measure ${ }^{\underline{5}}$ on $\mathscr{S}$ and the natural identification between $\mathfrak{H}$ and $\bigoplus_{s \in \mathscr{S}} \mathfrak{S}(s)$ respectively.

Given a diagonalization $(\nu, \mathfrak{S}, V)$, we would like to have a decomposition of $H$ of the form

$$
H=V^{-1} \int^{\oplus} \mathcal{H}(s) d \nu(s) V,
$$

where $\mathcal{H}$ is a $\nu$-measurable family of closed operators in $\mathfrak{S}$. This decomposition is an analogue of (2) in the direct integral setting. As we have seen, such a decomposition, in general, does not exist even in the direct sum case. It turns out, however, that this problem does not arise if we confine ourselves to exact diagonalizations that are singled out by the requirement that

$$
V^{-1} \mathcal{T}_{g}^{\nu, \mathfrak{S}} V \in \mathcal{A}(\mathfrak{X})
$$

for every $\nu$-measurable $\nu$-essentially bounded function $g$. It will be shown that, in contrast to direct sum decompositions, exact diagonalizations exist for every involutive set $\mathfrak{X} \subset L(\mathfrak{H})$ of pairwise commuting operators. Exactness condition (5) (which is an analogue of (3)) implies that $V H V^{-1}$ commutes with $\mathcal{T}_{g}^{\nu, S}$ for every $\nu$-measurable $\nu$-essentially bounded $g$ since, as shown above, $H$ commutes with all elements of $\mathcal{A}(\mathfrak{X})$. This allows us to apply the von Neumann's reduction theory ${ }^{6}$ (or, more precisely, its generalization 7.8 for the case of unbounded operators) to $V H V^{-1}$ and obtain decomposition (44) as a result. Moreover, given an exact diagonalization $(\nu, \mathfrak{S}, V)$ for $\mathfrak{X}$ and a decomposition of form (44), the symmetry preserving closed (resp., self-adjoint) extensions of $H$ are precisely the operators of the form

$$
V^{-1} \int^{\oplus} \tilde{\mathcal{H}}(s) d \nu(s) V
$$

where $\tilde{\mathcal{H}}$ is a $\nu$-measurable family of operators in $\mathfrak{S}$ such that $\tilde{\mathcal{H}}(s)$ is a closed (resp., self-adjoint) extension of the partial operator $\mathcal{H}(s)$ for $\nu$-a.e. $s \stackrel{9}{9}^{-}$Condition (5) is, as a rule, inconvenient for concrete applications because it involves the algebra $\mathcal{A}(\mathfrak{X})$ rather than the set $\mathfrak{X}$ itself. We shall see, however, that a simple exactness criterion similar to condition (E) can be obtained under very mild restrictions on the measure $\nu$ (Theorem IV.2). This generalized condition (E) can usually be easily verified for concrete examples.

The results formulated above suggest that, in general, one can find the symmetry preserving self-adjoint extensions of $H$ by doing the following steps:

(I) Find an exact diagonalization $(\nu, \mathfrak{S}, V)$ for $\mathfrak{X}$.

(II) Compute partial operators $\mathcal{H}(s)$ satisfying (4).

(III) Find self-adjoint extensions of the partial operators $\mathcal{H}(s)$.

In practice, the operator $H$ often comes as the closure of some non-closed operator $\check{H}$. While $\check{H}$ is usually given by some explicit formula, finding an explicit description of $H$ may be a difficult task. We shall see, however, that every symmetry of $\check{H}$ is also a symmetry of $H$ (Lemma III.1). Moreover, finding partial operators of $H$ can be effectively reduced to some computations involving $H$ (Proposition V.3). For this reason, the knowledge of $\check{H}$ is actually sufficient for doing steps (I)-(III) for $H$.

We apply the general construction described above to the three-dimensional model of an electron in the magnetic field of an infinitely thin solenoid. The Hamiltonian for this model is formally given by the differential expression

$$
\sum_{j=1}^{3}\left(-i \partial_{x_{j}}+\mathscr{A}_{j}(x)\right)^{2}
$$


where $x=\left(x_{1}, x_{2}, x_{3}\right) \in \mathbb{R}^{3}$ and the vector potential $\mathscr{A}=\left(\mathscr{A}_{1}, \mathscr{A}_{2}, \mathscr{A}_{3}\right)$ has the form

$$
\mathscr{A}_{1}(x)=-\frac{\phi x_{2}}{x_{1}^{2}+x_{2}^{2}}, \quad \mathscr{A}_{2}(x)=\frac{\phi x_{1}}{x_{1}^{2}+x_{2}^{2}}, \quad \mathscr{A}_{3}(x)=0 .
$$

Here, the real parameter $\phi$ is equal to the flux of the magnetic field through the solenoid divided by $2 \pi$.

This model was originally considered by Aharonov and Bohm $\underline{10}$. Self-adjoint extensions in the twodimensional variant of this model, as well as their eigenfunction expansions and corresponding scattering amplitudes, were analysed in Refs. 11 13.

The vector potential $\mathscr{A}$ is smooth on the domain $\mathscr{O}=\left\{\left(x_{1}, x_{2}, x_{3}\right) \in \mathbb{R}^{3}: x_{1}^{2}+x_{2}^{2}>0\right\}$, which is obtained by excluding the $x_{3}$-axis from $\mathbb{R}^{3}$. Hence, (6) naturally determines an operator $\mathscr{H}^{\phi}$ in the space $C^{\infty}(\mathscr{O})$ of smooth functions on $\mathscr{O}$,

$$
\begin{aligned}
\left(\mathscr{H}^{\phi} \Phi\right)(x)=\sum_{j=1}^{3}\left(-i \partial_{x_{j}}+\mathscr{A}_{j}(x)\right)^{2} \Phi(x)= & \\
= & \left(-\Delta+\frac{2 i \phi}{x_{1}^{2}+x_{2}^{2}}\left(x_{2} \partial_{x_{1}}-x_{1} \partial_{x_{2}}\right)+\frac{\phi^{2}}{x_{1}^{2}+x_{2}^{2}}\right) \Phi(x), \quad \Phi \in C^{\infty}(\mathscr{O}) .
\end{aligned}
$$

Restricting $\mathscr{H}^{\phi}$ to the space $C_{0}^{\infty}(\mathscr{O})$ of smooth functions on $\mathscr{O}$ with compact support and passing to $\Lambda$-equivalence classes, where $\Lambda$ is the Lebesgue measure on $\mathbb{R}^{3}$, we obtain a (non-closed) densely defined symmetric operator $\check{H}^{\phi}$ in $L_{2}\left(\mathbb{R}^{3}\right)$ :

$$
\begin{aligned}
& D_{\check{H}^{\phi}}=\left\{[\Phi]_{\Lambda}: \Phi \in C_{0}^{\infty}(\mathscr{O})\right\}, \\
& \breve{H}^{\phi}[\Phi]_{\Lambda}=\left[\mathscr{H}^{\phi} \Phi\right]_{\Lambda}, \quad \Phi \in C_{0}^{\infty}(\mathscr{O}),
\end{aligned}
$$

where $[\Phi]_{\Lambda}$ denotes the $\Lambda$-equivalence class corresponding to $\Phi$. We define the operator $H^{\phi}$ in $L_{2}\left(\mathbb{R}^{3}\right)$ as the closure of $\check{H}^{\phi}$,

$$
H^{\phi}=\overline{\bar{H}^{\phi}}
$$

Let $\mathcal{G}$ be the Abelian group of linear operators in $\mathbb{R}^{3}$ generated by translations along the $x_{3}$-axis and rotations around the $x_{3}$-axis. Given $G \in \mathcal{G}$, we denote by $T_{G}$ the unitary operator in $L_{2}\left(R^{3}\right)$ taking $\Psi$ to $\left[\Psi \circ G^{-1}\right]_{\Lambda}$ for any $\Psi \in L_{2}\left(\mathbb{R}^{3}\right)$. Clearly, $G \rightarrow T_{G}$ is a linear representation of $\mathcal{G}$ in $L_{2}\left(\mathbb{R}^{3}\right)$. It is straightforward to check that $\dot{H}^{\phi}$ (and, hence, $H^{\phi}$ ) commutes with $T_{G}$ for any $G \in \mathcal{G}$. The above abstract scheme can therefore be applied to $H=H^{\phi}$ and the set $\mathfrak{X}$ consisting of all $T_{G}$ with $G \in \mathcal{G}$. As a result, we shall describe all self-adjoint extensions of $H^{\phi}$ commuting with $T_{G}$ for any $G \in \mathcal{G}$ and find eigenfunction expansions for such extensions.

The paper is organized as follows. In Sec. II we formulate the results concerning self-adjoint extensions and eigenfunction expansions for the Aharonov-Bohm model. In Secs. [II general scheme of constructing symmetry preserving extensions outlined above. Sec. [III is devoted to preliminaries concerning the commutation properties of operators in Hilbert space. In Sec. [V] we establish the existence of exact diagonalizations and prove an exactness criterion, analogous to condition (E) discussed above. In Sec. $\mathrm{V}$ we consider direct integral decompositions for closed operators possessing a given set of symmetries and describe their symmetry preserving extensions. The aim of Secs. VI $\mathrm{X}$ is to derive eigenfunction expansions for the Aharonov-Bohm model. Sec. VI is concerned with self-adjoint extensions and eigenfunction expansions for one-dimensional Schrödinger operators. In Sec. VII, we treat the measurability questions for families of such operators. Secs. VI and VII provide a background for performing steps (I)-(III) for $H^{\phi}$ in Sec VIII] As a result, we represent symmetry preserving self-adjoint extensions of $H^{\phi}$ as direct integrals of suitable one-dimensional Schrödinger operators. In Sec IX, we study a special construction, called the direct integral of measures, which can be viewed as a generalization of direct products of measures and arises naturally in the spectral analysis of direct integrals of operators. In Sec. X] we combine the results of Secs. VI VIII, and IX to obtain the eigenfunction expansions of symmetry preserving self-adjoint extensions of $H^{\phi}$. In Appendices $\mathrm{A}$ and $\mathrm{B}$, we give the necessary background material concerning measure theory and direct integral decompositions of operators in Hilbert space respectively.

\section{FORMULATION OF RESULTS FOR THE AHARONOV-BOHM MODEL}

In this section, we give a precise description of self-adjoint extensions of $H^{\phi}$ commuting with $T_{G}$ for all $G \in \mathcal{G}$ and their eigenfunction expansions. The proofs of results stated below will be given in Sec. $\mathrm{X}$ 
Let $S=\mathbb{Z} \times \mathbb{R}$, where $\mathbb{Z}$ is the set of integers. For $\phi \in \mathbb{R}$, let the subset $A^{\phi}$ of $S$ be given by

$$
A^{\phi}=\{(m, p) \in S:|m+\phi|<1\} .
$$

For every Bore! $\underline{14}^{\underline{4}}$ real function $\theta$ on $A^{\phi}$, we shall define a map $\mathcal{W}_{\theta}^{\phi}$ on $S \times \mathbb{R}$ such that

(a) For every $s \in S$ and $E \in \mathbb{R}, \mathcal{W}_{\theta}^{\phi}(s, E)$ is a smooth complex function on $\mathscr{O}$ that is locally squareintegrable on $\mathbb{R}^{3}$.

(b) The equalities $\frac{15}{}$

$$
\begin{aligned}
& \mathscr{H}^{\phi} \mathcal{W}_{\theta}^{\phi}(s, E)=\left(E+p^{2}\right) \mathcal{W}_{\theta}^{\phi}(s, E), \\
& -i \partial_{x_{3}} \mathcal{W}_{\theta}^{\phi}(s, E \mid x)=p \mathcal{W}_{\theta}^{\phi}(s, E \mid x), \\
& -i\left(x_{1} \partial_{x_{2}}-x_{2} \partial_{x_{1}}\right) \mathcal{W}_{\theta}^{\phi}(s, E \mid x)=m \mathcal{W}_{\theta}^{\phi}(s, E \mid x)
\end{aligned}
$$

hold for every $s=(m, p) \in S, E \in \mathbb{R}$, and $x=\left(x_{1}, x_{2}, x_{3}\right) \in \mathscr{O}$.

Furthermore, we shall define a positive Borel measure $M_{\theta}^{\phi}$ on $S \times \mathbb{R}$ such that the following statement is valid.

Proposition II.1. Let $\phi \in \mathbb{R}$ and $\theta$ be a Borel real function on $A^{\phi}$. Then there is a unique unitary operator $W: L_{2}\left(\mathbb{R}^{3}\right) \rightarrow L_{2}\left(S \times \mathbb{R}, M_{\theta}^{\phi}\right)$ such that

$$
(W \Psi)(s, E)=\int \overline{\mathcal{W}_{\theta}^{\phi}(s, E \mid x)} \Psi(x) d x, \quad \Psi \in L_{2}^{c}\left(\mathbb{R}^{3}\right),
$$

for $M_{\theta}^{\phi}$-a.e. $(s, E)$, where the bar means complex conjugation and $L_{2}^{c}\left(\mathbb{R}^{3}\right)$ is the subspace of $L_{2}\left(\mathbb{R}^{3}\right)$ consisting of all its elements vanishing $\Lambda$-a.e. outside some compact subset of $\mathbb{R}^{3}$.

Condition (b) means that $\mathcal{W}_{\theta}^{\phi}(m, s)$ is a joint generalized eigenfunction of operator (6) and $x_{3}$ components of momentum and angular momentum for every $s \in S$ and $E \in \mathbb{R}$. Proposition 【I.1 states that these eigenfunctions constitute a complete set and can be used to expand square-integrable functions on $\mathbb{R}^{3}$. We shall see (Theorem [I.5) that all such eigenfunction expansions correspond to symmetry preserving self-adjoint extensions of $H^{\phi}$, but first we give precise definitions of $\mathcal{W}_{\theta}^{\phi}$ and $M_{\theta}^{\phi}$.

It follows from the second and third equalities in condition (b) that

$$
\mathcal{W}_{\theta}^{\phi}(s, E \mid x)=\frac{e^{i p x_{3}}}{2 \pi \sqrt{r_{x}}}\left(\frac{x_{1}+i x_{2}}{r_{x}}\right)^{m} \mathcal{J}_{\theta}^{\phi}\left(s, E \mid r_{x}\right)
$$

for every $s=(m, p) \in S, E \in \mathbb{R}$, and $x \in \mathscr{O}$, where $r_{x}=\sqrt{x_{1}^{2}+x_{2}^{2}}$ and $\mathcal{J}_{\theta}^{\phi}(s, E)$ is some smooth function on $\mathbb{R}_{+}=(0, \infty)$ (note that $x_{1} \partial_{x_{2}}-x_{2} \partial_{x_{1}}$ and $r_{x}^{-m}\left(x_{1}+i x_{2}\right)^{m}$ correspond to $\partial_{\varphi}$ and $e^{i m \varphi}$ respectively in the polar coordinates). For future convenience, we write the factor $\left(2 \pi \sqrt{r_{x}}\right)^{-1}$ separately rather than subsume it into $\mathcal{J}_{\theta}^{\phi}(s, E)$. The first equality in condition (b) is fulfilled if and only if $\mathcal{J}_{\theta}^{\phi}(s, E)$ satisfies the one-dimensional Schrödinger equation

$$
-\partial_{r}^{2} \mathcal{J}_{\theta}^{\phi}(s, E \mid r)+\frac{(m+\phi)^{2}-1 / 4}{r^{2}} \mathcal{J}_{\theta}^{\phi}(s, E \mid r)=E \mathcal{J}_{\theta}^{\phi}(s, E \mid r), \quad r \in \mathbb{R}_{+},
$$

for every $s=(m, p) \in S$ and $E \in \mathbb{R}$.

To define $\mathcal{J}_{\theta}^{\phi}$ explicitly, we introduce some additional notation. For any $E, \kappa \in \mathbb{R}$, we define the function $u^{\kappa}(E)$ on $\mathbb{R}_{+}$by the relation

$$
u^{\kappa}(E \mid r)=r^{1 / 2+\kappa} \mathcal{X}_{\kappa}\left(r^{2} E\right), \quad r \in \mathbb{R}_{+},
$$

where the entire function $\mathcal{X}_{\kappa}$ is given by

$$
\mathcal{X}_{\kappa}(\zeta)=\frac{1}{2^{\kappa}} \sum_{n=0}^{\infty} \frac{(-1)^{n} \zeta^{n}}{\Gamma(\kappa+n+1) n ! 2^{2 n}}, \quad \zeta \in \mathbb{C} .
$$

For $-1<\kappa<1, \vartheta \in \mathbb{R}$, and $E \in \mathbb{R}$, we define the function $u_{\vartheta}^{\kappa}(E)$ on $\mathbb{R}_{+}$by setting

$$
u_{\vartheta}^{\kappa}(E)=\frac{u^{\kappa}(E) \sin \left(\vartheta+\vartheta_{\kappa}\right)-u^{-\kappa}(E) \sin \left(\vartheta-\vartheta_{\kappa}\right)}{\sin \pi \kappa}, \quad 0<|\kappa|<1,
$$


and

$$
u_{\vartheta}^{0}(E \mid r)=\lim _{\kappa \rightarrow 0} u_{\vartheta}^{\kappa}(E \mid r)=u^{0}(E \mid r) \cos \vartheta+\frac{2}{\pi}\left[\left(\ln \frac{r}{2}+\gamma\right) u^{0}(E \mid r)-\sqrt{r} \mathcal{Y}\left(r^{2} E\right)\right] \sin \vartheta, \quad r \in \mathbb{R}_{+},
$$

where

$$
\vartheta_{\kappa}=\frac{\pi \kappa}{2}
$$

the entire function $\mathcal{Y}$ is given by

$$
\mathcal{Y}(\zeta)=\sum_{n=1}^{\infty} \frac{(-1)^{n} c_{n}}{(n !)^{2} 2^{2 n}} \zeta^{n}, \quad c_{n}=\sum_{j=1}^{n} \frac{1}{j},
$$

and $\gamma=\lim _{n \rightarrow \infty}\left(c_{n}-\ln n\right)=0,577 \ldots$ is the Euler constant. ${ }^{16}$ We now define $\mathcal{J}_{\theta}^{\phi}$ by setting

$$
\mathcal{J}_{\theta}^{\phi}(s, E)=\left\{\begin{array}{cc}
u^{|m+\phi|}(E), & s \in S \backslash A^{\phi} \\
u_{\theta(s)}^{m+\phi}(E), & s \in A^{\phi}
\end{array}\right.
$$

for every $s=(m, p) \in S$ and $E \in \mathbb{R}$.

The function $\mathcal{X}_{\kappa}$ is closely related to Bessel functions: for $\zeta \neq 0$, we have

$$
\mathcal{X}_{\kappa}(\zeta)=\zeta^{-\kappa / 2} J_{\kappa}\left(\zeta^{1 / 2}\right)
$$

where $J_{\kappa}$ is the Bessel function of the first kind of order $\kappa$. Since $J_{\kappa}$ satisfies the Bessel equation, it follows that

$$
-\partial_{r}^{2} u^{ \pm \kappa}(E \mid r)+\frac{\kappa^{2}-1 / 4}{r^{2}} u^{ \pm \kappa}(E \mid r)=E u^{ \pm \kappa}(E \mid r), \quad r \in \mathbb{R}_{+}
$$

for every $E \neq 0$. By continuity, this is true for $E=0$ as well. Moreover, (21) is satisfied for $u_{\vartheta}^{\kappa}(E)$ in place of $u^{ \pm \kappa}(E)$ for every $-1<\kappa<1, E \in \mathbb{R}$, and $\vartheta \in \mathbb{R}$ (this obviously follows from (15) for $0<|\kappa|<1$; for $\kappa=0$, we can express $u_{\vartheta}^{0}(E \mid r)$ in terms of the Bessel functions $J_{0}$ and $Y_{0}$ by means of the equality ${ }^{17} \pi Y_{0}(\zeta)=2(\gamma+\ln (\zeta / 2)) J_{0}(\zeta)-2 \mathcal{Y}\left(\zeta^{2}\right)$ and make use of the Bessel equation). In view of (19), we conclude that (12) holds for every $s \in S$ and $E \in \mathbb{R}$. It follows immediately from (11), (13), (15), (16), and (19) that $\mathcal{W}_{\theta}^{\phi}(s, E)$ is locally square-integrable on $\mathbb{R}^{3}$ for every $s \in S$ and $E \in \mathbb{R}$. Thus, both conditions (a) and (b) are fulfilled for the map $\mathcal{W}_{\theta}^{\phi}$ defined by (11) and (19).

It should be noted that equation (12) and condition (a) determine $\mathcal{J}_{\theta}^{\phi}(s, E)$ uniquely up to a numerical factor for $s \in S \backslash A^{\phi}$. At the same time, if $s \in A^{\phi}$, then there are two linearly independent solutions of (12) compatible with (a) for every $E \in \mathbb{R}$. According to (19), different choices of the function $\theta$ on $A^{\phi}$ allow us to pick different solutions of (12) and, consequently, lead to different eigenfunction expansions for operator (6).

We now proceed to define the measure $M_{\theta}^{\phi}$. The above definition of $\mathcal{W}_{\theta}^{\phi}$ involves the generalized eigenfunctions $u^{\kappa}(E)$ and $u_{\vartheta}^{\kappa}(E)$ of the one-dimensional Schrödinger operator

$$
-\partial_{r}^{2}+\frac{\kappa^{2}-1 / 4}{r^{2}}
$$

Similarly, $M_{\theta}^{\phi}$ will be constructed from one-dimensional measures $\mathcal{V}_{\kappa}$ and $\mathcal{V}_{\kappa, \vartheta}$ entering eigenfunction expansions associated with differential expression (22) (see Propositions VI.3 and VI.4). For $\kappa \in \mathbb{R}$, the positive Borel measure $\mathcal{V}_{\kappa}$ on $\mathbb{R}$ is given by

$$
d \mathcal{V}_{\kappa}(E)=\frac{1}{2} \Theta(E) E^{|\kappa|} d E
$$

where $\Theta$ is the Heaviside function, i.e., $\Theta(E)=1$ for $E \geq 0$ and $\Theta(E)=0$ for $E<0$. Given $-1<\kappa<1$ and $\vartheta \in \mathbb{R}$, we define the positive Borel measure $\mathcal{V}_{\kappa, \vartheta}$ on $\mathbb{R}$ as follows. If $0<|\kappa|<1$, we set

$$
\mathcal{V}_{\kappa, \vartheta}=\left\{\begin{array}{cc}
\tilde{\mathcal{V}}_{\kappa, \vartheta}, & \vartheta \in\left[-\left|\vartheta_{\kappa}\right|,\left|\vartheta_{\kappa}\right|\right]+\pi \mathbb{Z}, \\
\frac{\pi \sin \pi \kappa\left|E_{\kappa, \vartheta}\right|}{2 \kappa \sin \left(\vartheta+\vartheta_{\kappa}\right) \sin \left(\vartheta-\vartheta_{\kappa}\right)} \delta_{E_{\kappa, \vartheta}}+\tilde{\mathcal{V}}_{\kappa, \vartheta}, & \vartheta \in\left(\left|\vartheta_{\kappa}\right|, \pi-\left|\vartheta_{\kappa}\right|\right)+\pi \mathbb{Z},
\end{array}\right.
$$


where $\vartheta_{\kappa}$ is defined by (17), the positive Borel measure $\tilde{\mathcal{V}}_{\kappa, \vartheta}$ on $\mathbb{R}$ is given by

$$
d \tilde{\mathcal{V}}_{\kappa, \vartheta}(E)=\frac{1}{2} \frac{\Theta(E) \sin ^{2} \pi \kappa}{E^{-\kappa} \sin ^{2}\left(\vartheta+\vartheta_{\kappa}\right)-2 \cos \pi \kappa \sin \left(\vartheta+\vartheta_{\kappa}\right) \sin \left(\vartheta-\vartheta_{\kappa}\right)+E^{\kappa} \sin ^{2}\left(\vartheta-\vartheta_{\kappa}\right)} d E
$$

and $\delta_{E_{\kappa, \vartheta}}$ is the Dirac measure at the point

$$
E_{\kappa, \vartheta}=-\left(\frac{\sin \left(\vartheta+\vartheta_{\kappa}\right)}{\sin \left(\vartheta-\vartheta_{\kappa}\right)}\right)^{1 / \kappa}
$$

For $\kappa=0$, the measure $\mathcal{V}_{\kappa, \vartheta}$ is defined by taking the limit $\kappa \rightarrow 0$ in formulas (24), (25), and (26). This yields

$$
\mathcal{V}_{0, \vartheta}=\left\{\begin{array}{cc}
\tilde{\mathcal{V}}_{0, \vartheta}, & \vartheta \in \pi \mathbb{Z} \\
\frac{\pi^{2}\left|E_{0, \vartheta}\right|}{2 \sin ^{2} \vartheta} \delta_{E_{0, \vartheta}}+\tilde{\mathcal{V}}_{0, \vartheta}, & \vartheta \notin \pi \mathbb{Z}
\end{array}\right.
$$

where

$$
E_{0, \vartheta}=-e^{\pi \cot \vartheta}
$$

and the positive Borel measure $\tilde{\mathcal{V}}_{0, \vartheta}$ on $\mathbb{R}$ is given by

$$
d \tilde{\mathcal{V}}_{0, \vartheta}(E)=\frac{1}{2} \frac{\Theta(E)}{(\cos \vartheta-\ln E \sin \vartheta / \pi)^{2}+\sin ^{2} \vartheta} d E .
$$

The next lemma shows, in particular, that the measure $\mathcal{V}_{\kappa, \vartheta}$ depends continuously on both $\kappa$ and $\vartheta$.

Lemma II.2. Let $F$ be a bounded (continuous) Borel function on $\mathbb{R}$ with compact support. Then $(\kappa, \vartheta) \rightarrow$ $\int F(E) d \mathcal{V}_{\kappa, \vartheta}(E)$ is a Borel (resp., continuous) function on $(-1,1) \times \mathbb{R}$ that is bounded on $[-\alpha, \alpha] \times \mathbb{R}$ for every $0 \leq \alpha<1$.

Proof. See Theorem 4 in Ref. 18.

Given a Borel real function $\theta$ on $A^{\phi}$, let $\mu_{\theta}^{\phi}$ be the measure-valued map on $S$ such that

$$
\mu_{\theta}^{\phi}(s)=\left\{\begin{array}{cc}
\mathcal{V}_{|m+\phi|}, & s \in S \backslash A^{\phi} \\
\mathcal{V}_{m+\phi, \theta(s)}, & s \in A^{\phi}
\end{array}\right.
$$

for every $s=(m, p) \in S$.

The next statement follows immediately from Lemma Џ.2.

Lemma II.3. Let $\phi \in \mathbb{R}$ and $\theta$ be a Borel real function on $A^{\phi}$. For any compact set $K \subset \mathbb{R}, s \rightarrow \mu_{\theta}^{\phi}(s \mid K)$ is a Borel function on $S$ that is bounded on every compact subset of $S$.

Let $\varrho$ be the counting measure on $\mathbb{Z}$. We define the positive Borel measure $\nu_{0}$ on $S=\mathbb{Z} \times \mathbb{R}$ by setting $\nu_{0}=\varrho \times \lambda$, where $\lambda$ is the Lebesgue measure on $\mathbb{R}$. For any $\nu_{0}$-integrable $f$, the function $p \rightarrow f(m, p)$ is integrable for every $m \in \mathbb{Z}$ and we have

$$
\int_{S} f(m, p) d \nu_{0}(m, p)=\sum_{m \in \mathbb{Z}} \int_{-\infty}^{\infty} f(m, p) d p .
$$

Proposition II.4. Let $\phi \in \mathbb{R}$ and $\theta$ be a Borel real function on $A^{\phi}$. Then there is a unique positive Borel measure $M$ on $S \times \mathbb{R}$ such that

$$
M\left(K^{\prime} \times K\right)=\int_{K^{\prime}} \mu_{\theta}^{\phi}(s \mid K) d \nu_{0}(s)
$$

for every compact sets $K^{\prime} \subset S$ and $K \subset \mathbb{R}$. If $f$ is an $M$-integrable complex function, then the function $E \rightarrow f(s, E)$ is $\mu_{\theta}^{\phi}(s)$-integrable for $\nu_{0}$-a.e. $s$, the function $s \rightarrow \int f(s, E) d \mu_{\theta}^{\phi}(s \mid E)$ is $\nu_{0}$-integrable, and

$$
\int f(s, E) d M(s, E)=\int d \nu_{0}(s) \int f(s, E) d \mu_{\theta}^{\phi}(s \mid E) .
$$


Note that the right-hand side of (31) is well-defined in view of Lemma I.3. Given $\phi \in \mathbb{R}$ and a Borel real function $\theta$ on $A^{\phi}$, we let $M_{\theta}^{\phi}$ denote the measure $M$ satisfying the conditions of Proposition $\amalg .4$ We thus have explicitly defined all entities entering Proposition II.1.

It is worth mentioning that the structure of the set $A^{\phi}$ depends on whether $\phi \in \mathbb{Z}$ or not. If $\phi \in \mathbb{Z}$, then we have $A^{\phi}=\{-\phi\} \times \mathbb{R}$ and defining a Borel function on $A^{\phi}$ amounts to defining a single Borel function on $\mathbb{R}$. On the other hand, if $\phi \notin \mathbb{Z}$, then $A^{\phi}=\left\{m_{\phi}, m_{\phi}+1\right\} \times \mathbb{R}$, where $m_{\phi} \in \mathbb{Z}$ is such that $-\phi-1<m_{\phi}<-\phi$, and every Borel function on $A^{\phi}$ is determined by two Borel functions on $\mathbb{R}$. Using functions on $A^{\phi}$ instead of functions on $\mathbb{R}$ allows us to treat both cases uniformly.

Given $\phi \in \mathbb{R}$ and a Borel real function $\theta$ on $A^{\phi}$, the operator $W$ satisfying the conditions of Proposition $\amalg$.1 will be denoted by $W_{\theta}^{\phi}$. Let the function $\mathfrak{f}$ on $S \times \mathbb{R}$ be defined by the relation

$$
\mathfrak{f}(m, p ; E)=p^{2}+E .
$$

We define the self-adjoint operator $H_{\theta}^{\phi}$ in $L_{2}\left(\mathbb{R}^{3}\right)$ by setting

$$
H_{\theta}^{\phi}=\left(W_{\theta}^{\phi}\right)^{-1} \mathcal{T}_{\mathfrak{f}}^{M} W_{\theta}^{\phi}
$$

where $M=M_{\theta}^{\phi}$ and $\mathcal{T}_{\mathfrak{f}}^{M}$ is the operator of multiplication by $\mathfrak{f}$ in $L_{2}(S \times \mathbb{R}, M)$ (see Sec. A 7 ).

Let $\Phi \in C_{0}^{\infty}(\mathscr{O}), \Psi=[\Phi]_{\Lambda}$, and $h_{\Psi}(s, E)$ be the right-hand side of (10). In view of (17) and the first equality in condition (b), integrating by parts yields

$$
h_{\breve{H}^{\phi} \Psi}(s, E)=\int \overline{\mathcal{W}_{\theta}^{\phi}(s, E \mid x)}\left(\mathscr{H}^{\phi} \Phi\right)(x) d x=\int \overline{\left(\mathscr{H}^{\phi} \mathcal{W}_{\theta}^{\phi}(s, E)\right)(x)} \Psi(x) d x=\mathfrak{f}(s, E) h_{\Psi}(s, E)
$$

for every $s \in S$ and $E \in \mathbb{R}$. It follows that $W_{\theta}^{\phi} \check{H}^{\phi} \Psi=\mathcal{T}_{\mathfrak{f}}^{M} W_{\theta}^{\phi} \Psi$ for every $\Psi \in D_{\check{H}^{\phi}}$, i.e., $H_{\theta}^{\phi}$ is a self-adjoint extension of $\check{H}^{\phi}$ and, hence, of $H^{\phi}$.

Further, given $\alpha, \beta \in \mathbb{R}$, let $G_{\alpha \beta} \in \mathcal{G}$ be defined by the relation

$$
G_{\alpha \beta} x=\left(x_{1} \cos \alpha-x_{2} \sin \alpha, x_{1} \sin \alpha+x_{2} \cos \alpha, x_{3}+\beta\right),
$$

where $x=\left(x_{1}, x_{2}, x_{3}\right) \in \mathbb{R}^{3}$. It follows from (11) that

$$
\mathcal{W}_{\theta}^{\phi}\left(s, E \mid G_{\alpha \beta} x\right)=e^{i m \alpha+i p \beta} \mathcal{W}_{\theta}^{\phi}(s, E \mid x), \quad x \in \mathscr{O},
$$

for every $s=(m, p) \in S$ and $E \in \mathbb{R}$. Let $\Psi \in L_{2}^{c}\left(\mathbb{R}^{3}\right)$. Setting $\tilde{\Psi}=T_{G_{\alpha \beta}} \Psi$, we obtain

$$
h_{\tilde{\Psi}}(s, E)=\int \overline{\mathcal{W}_{\theta}^{\phi}(s, E \mid x)} \Psi\left(G_{\alpha \beta}^{-1} x\right) d x=g_{\alpha \beta}(s, E) h_{\Psi}(s, E)
$$

for every $s \in S$ and $E \in \mathbb{R}$, where $g_{\alpha \beta}$ is the function $(m, p ; E) \rightarrow e^{-i m \alpha-i p \beta}$ on $S \times \mathbb{R}$. As $L_{2}^{c}\left(\mathbb{R}^{3}\right)$ is dense in $L_{2}\left(\mathbb{R}^{3}\right)$, this implies that

$$
T_{G_{\alpha \beta}}=\left(W_{\theta}^{\phi}\right)^{-1} \mathcal{T}_{g_{\alpha \beta}}^{M} W_{\theta}^{\phi}
$$

Since every element of $\mathcal{G}$ is equal to $G_{\alpha \beta}$ for some $\alpha, \beta \in \mathbb{R}$ and $\mathcal{T}_{\mathfrak{f}}^{M}$ commutes with $\mathcal{T}_{g_{\alpha \beta}}^{M}$, it follows from (34) that $H_{\theta}^{\phi}$ commutes with $T_{G}$ for all $G \in \mathcal{G}$.

Let $\theta$ and $\tilde{\theta}$ be Borel real functions on $A^{\phi}$ such that $\theta(s)-\tilde{\theta}(s) \in \pi \mathbb{Z}$ for $\nu_{0}$-a.e. $s \in A^{\phi}$. By (24)(29), we have $\mathcal{V}_{\kappa, \vartheta+\pi n}=\mathcal{V}_{\kappa, \vartheta}$ for every $-1<\kappa<1, \vartheta \in \mathbb{R}$, and $n \in \mathbb{Z}$. In view of (30), it follows that $\mu_{\theta}^{\phi}(s)=\mu_{\tilde{\theta}}^{\phi}(s)$ for $\nu_{0}$-a.e. $s$. The uniqueness statement of Proposition II.4 therefore implies that $M_{\theta}^{\phi}=M_{\tilde{\theta}}^{\phi}$. By (15) and (16), we have $u_{\vartheta+\pi n}^{\kappa}(z)=e^{i \pi n} u_{\vartheta}^{\kappa}(z)$ for every $-1<\kappa<1, \vartheta \in \mathbb{R}, z \in \mathbb{C}$, and $n \in \mathbb{Z}$. It follows from (11) and (19) that

$$
\mathcal{W}_{\tilde{\theta}}^{\phi}(s, E \mid x)=f_{\theta \tilde{\theta}}(s, E) \mathcal{W}_{\theta}^{\phi}(s, E \mid x)
$$

for every $s \in S \backslash N, E \in \mathbb{R}$, and $x \in \mathscr{O}$, where $N=\left\{s \in A^{\phi}: \theta(s)-\tilde{\theta}(s) \notin \pi \mathbb{Z}\right\}$ is a $\nu_{0}$-null set and the Borel function $f_{\theta \tilde{\theta}}$ on $S \times \mathbb{R}$ is given by

$$
f_{\theta \tilde{\theta}}(s, E)=\left\{\begin{array}{cc}
1, & s \in S \backslash A^{\phi}, \\
e^{i(\tilde{\theta}(s)-\theta(s))}, & s \in A^{\phi} .
\end{array}\right.
$$

It easily follows from Proposition $\llbracket .4$ that $N \times \mathbb{R}$ is an $M$-null set, where $M=M_{\theta}^{\phi}=M_{\tilde{\theta}}^{\phi}$, and, therefore, $f_{\theta \tilde{\theta}}(s, E)= \pm 1$ for $M$-a.e. $(s, E)$. Proposition II.1 and formula (36) hence imply that $W_{\tilde{\theta}}^{\phi}=\mathcal{T}_{f_{\theta \tilde{\theta}}}^{M} W_{\theta}^{\phi}$. In view of (34), we conclude that $H_{\theta}^{\phi}=H_{\tilde{\theta}}^{\phi}$.

Thus, assuming Propositions II.4 and II.1 we have proved the next statement in one direction. 
Theorem II.5. Let $\phi \in \mathbb{R}$. For every Borel real function $\theta$ on $A^{\phi}$, the operator $H_{\theta}^{\phi}$ is a self-adjoint extension of $H^{\phi}$ commuting with $T_{G}$ for any $G \in \mathcal{G}$. Conversely, every self-adjoint extension of $H^{\phi}$ commuting with $T_{G}$ for any $G \in \mathcal{G}$ is equal to $H_{\theta}^{\phi}$ for some Borel real function $\theta$ on $A^{\phi}$. Given Borel real functions $\theta$ and $\tilde{\theta}$ on $A^{\phi}$, we have $H_{\theta}^{\phi}=H_{\tilde{\theta}}^{\phi}$ if and only if $\theta(s)-\tilde{\theta}(s) \in \pi \mathbb{Z}$ for $\nu_{0}$-a.e. $s \in A^{\phi}$.

\section{COMMUTATION OF OPERATORS AND VON NEUMANN ALGEBRAS}

In this section, we give some background material for the treatment of diagonalizations in Sec. IV] It should be noted that, as far as diagonalizations are concerned, the conditions imposed on $\mathfrak{X}$ in Introduction are excessively restrictive. In fact, it suffices to assume (and we do so in this section and Sec. IVV) that $\mathfrak{X}$ is an arbitrary set of closed densely defined operators rather than an involutive subset of $L(\mathfrak{H})$. In Sec. $\mathrm{V}$. however, where the reduction by symmetries is treated, the assumptions on $\mathfrak{X}$ are the same as in Introduction.

Lemma III.1. Let $\mathfrak{H}$ be a Hilbert space, $T \in L(\mathfrak{H})$, and $R$ be an operator in $\mathfrak{H}$ commuting with $T$. If $R$ is densely defined, then $R^{*}$ commutes with $T^{*}$. If $R$ is closable, then the closure $\bar{R}$ of $R$ commutes with $T$.

Proof. Suppose $R$ is densely defined. Let $\Psi \in D_{R^{*}}$ and $\Phi=R^{*} \Psi$. Then we have $\left\langle R \Psi^{\prime}, \Psi\right\rangle=\left\langle\Psi^{\prime}, \Phi\right\rangle$ for any $\Psi^{\prime} \in D_{R}$. Hence, we obtain

$$
\left\langle R \Psi^{\prime}, T^{*} \Psi\right\rangle=\left\langle R T \Psi^{\prime}, \Psi\right\rangle=\left\langle T \Psi^{\prime}, \Phi\right\rangle=\left\langle\Psi^{\prime}, T^{*} \Phi\right\rangle, \quad \Psi^{\prime} \in D_{R} .
$$

This means that $T^{*} \Psi \in D_{R^{*}}$ and $R^{*} T^{*} \Psi=T^{*} R^{*} \Psi$, i.e., $R^{*}$ commutes with $T^{*}$.

Suppose now that $R$ is closable. Let $\Psi \in D_{\bar{R}}$. Then there is a sequence $\Psi_{n} \in D_{R}$ such that $\Psi_{n} \rightarrow \Psi$ and $R \Psi_{n} \rightarrow \bar{R} \Psi$ in $\mathfrak{H}$. Since $R$ commutes with $T$, we have $T \Psi_{n} \in D_{R}$ for all $n$. The continuity of $T$ implies that $T \Psi_{n} \rightarrow T \Psi$ and $R T \Psi_{n}=T R \Psi_{n} \rightarrow T \bar{R} \Psi$. This means that $T \Psi \in D_{\bar{R}}$ and $\bar{R} T \Psi=T \bar{R} \Psi$.

Given a set $\mathfrak{X}$ of closed densely defined operators in a Hilbert space $\mathfrak{H}$, let $\mathfrak{X}^{\prime}$ denote its commutant, i.e., the subalgebra of $L(\mathfrak{H})$ consisting of all operators commuting with every element of $\mathfrak{X}$. Let $\mathfrak{X}^{*}$ be the set consisting of the adjoints of the elements of $\mathfrak{X}$. By Lemma III.1 we have

$$
\left(\mathfrak{X}^{\prime}\right)^{*}=\left(\mathfrak{X}^{*}\right)^{\prime} .
$$

The set $\mathfrak{X}$ is involutive if and only if $\mathfrak{X}^{*}=\mathfrak{X}$.

Recall 19 that a subalgebra $\mathcal{M}$ of $L(\mathfrak{H})$ is called a von Neumann algebra if it is involutive and coincides with its bicommutant $\mathcal{M}^{\prime \prime}$. By the well-known von Neumann's bicommutant theorem (see, e. g., Ref. 19, Sec. I.3.4, Corollaire 2), an involutive subalgebra $\mathcal{M}$ of $L(\mathfrak{H})$ is a von Neumann algebra if and only if it contains the identity operator and is closed in the strong operator topology. It follows from (37) that $\mathfrak{X}^{\prime}$ is an involutive subalgebra of $L(\mathfrak{H})$ for any involutive set $\mathfrak{X}$ of closed densely defined operators in $\mathfrak{H}$. Moreover, it is easy to show (see Lemma 1 in Ref. (4) that $\mathfrak{X}^{\prime}$ is always strongly closed and, therefore, is a von Neumann algebra for involutive $\mathfrak{X}$ by the bicommutant theorem.

A closed densely defined operator $T$ in $\mathfrak{H}$ is called affiliated with a von Neumann algebra $\mathcal{M}$ if $T$ commutes with every element of $\mathcal{M}^{\prime}$. If $\mathfrak{X}$ is a set of closed densely defined operators in $\mathfrak{H}$, then every element of $\mathfrak{X}$ is obviously affiliated with the algebra $\mathcal{A}(\mathfrak{X})=\left(\mathfrak{X} \cup \mathfrak{X}^{*}\right)^{\prime \prime}$. As shown by the next lemma, $\mathcal{A}(\mathfrak{X})$ is actually the smallest von Neumann algebra with this property.

Lemma III.2. Let $\mathfrak{X}$ be a set of closed densely defined operators in a Hilbert space $\mathfrak{H}$ and $\mathcal{M}$ be a von Neumann algebra in $\mathfrak{H}$. Then $\mathcal{A}(\mathfrak{X}) \subset \mathcal{M}$ if and only if every operator in $\mathfrak{X}$ is affiliated with $\mathcal{M}$.

Proof. If every element of $\mathfrak{X}$ is affiliated with $\mathcal{M}$, then $\mathcal{M}^{\prime} \subset \mathfrak{X}^{\prime}$, whence $\mathcal{M}^{\prime} \subset\left(\mathfrak{X}^{*}\right)^{\prime}$ by (37). It follows that $\mathcal{M}^{\prime} \subset \mathfrak{X}^{\prime} \cap\left(\mathfrak{X}^{*}\right)^{\prime}=\left(\mathfrak{X} \cup \mathfrak{X}^{*}\right)^{\prime}$ and, hence, $\mathcal{A}(\mathfrak{X}) \subset \mathcal{M}^{\prime \prime}=\mathcal{M}$. Conversely, if $\mathcal{A}(\mathfrak{X}) \subset \mathcal{M}$, then $\mathcal{M}^{\prime} \subset\left(\mathfrak{X} \cup \mathfrak{X}^{*}\right)^{\prime} \subset \mathfrak{X}^{\prime}$ and, hence, every element of $\mathfrak{X}$ is affiliated with $\mathcal{M}$.

The algebra $\mathcal{A}(\mathfrak{X})$ will be called the von Neumann algebra generated by $\mathfrak{X}$, and $\mathfrak{X}$ will be referred to as a set of generators of $\mathcal{A}(\mathfrak{X})$. If $\mathfrak{X} \subset L(\mathfrak{H})$, then $\mathcal{A}(\mathfrak{X})$ is just the smallest von Neumann algebra containing $\mathfrak{X}$. By Lemma III.2, a closed densely defined operator $T$ is affiliated with a von Neumann algebra $\mathcal{M}$ if and only if $\mathcal{A}(T) \subset \mathcal{M}$ (here and subsequently, we write $\mathcal{A}(T)$ instead of $\mathcal{A}(\{T\}$ ), where $\{T\}$ is the one-element set containing $T$ ).

Let $\mathfrak{X} \subset L(\mathfrak{H})$ be an involutive set of pairwise commuting operators. Then $\mathfrak{X} \subset \mathfrak{X}^{\prime}$ and, hence, $\mathcal{A}(\mathfrak{X}) \subset \mathfrak{X}^{\prime}$ because $\mathfrak{X}^{\prime}$ is a von Neumann algebra. As $\mathfrak{X}^{\prime}=\mathcal{A}(\mathfrak{X})^{\prime}$, it follows that $\mathcal{A}(\mathfrak{X})$ is Abelian.

We say that two sets $\mathfrak{X}$ and $\mathfrak{Y}$ of closed densely defined operators in $\mathfrak{H}$ are equivalent if $\mathcal{A}(\mathfrak{X})=\mathcal{A}(\mathfrak{Y})$. We say that $\mathfrak{X}$ is equivalent to a closed densely defined operator $T$ if $\mathfrak{X}$ is equivalent to the one-element set $\{T\}$. 
Remark III.3. If $\mathfrak{X} \subset L(\mathfrak{H})$ is an involutive set, then the smallest strongly closed algebra containing $\mathfrak{X}$ and the identity operator in $\mathfrak{H}$ is obviously a von Neumann algebra and, hence, coincides with $\mathcal{A}(\mathfrak{X})$. The above definition of $\mathcal{A}(\mathfrak{X})$ therefore complies with that used in Introduction.

Given a spectral measure $\mathcal{E}$ (see Sec. $\mathrm{A} 8$ ), we denote by $\mathcal{P}_{\mathcal{E}}$ the set of all operators $\mathcal{E}(A)$, where $A$ is an $\mathcal{E}$-measurable set. As the elements of $\mathcal{P}_{\mathcal{E}}$ pairwise commute, the algebra $\mathcal{A}\left(\mathcal{P}_{\mathcal{E}}\right)$ is Abelian. For an $\mathcal{E}$-measurable complex function $g$, we let $J_{g}^{\mathcal{E}}$ denote the integral of $g$ with respect to $\mathcal{E}$ (see Sec. A 8).

Lemma III.4. Let $\mathfrak{H}$ be a separable Hilbert space and $\mathcal{E}$ be a spectral measure in $\mathfrak{H}$. Then the following statements hold:

1. The algebra $\mathcal{A}\left(\mathcal{P}_{\mathcal{E}}\right)$ coincides with the set of all $J_{g}^{\mathcal{E}}$, where $g$ is an $\mathcal{E}$-measurable $\mathcal{E}$-essentially bounded complex function.

2. A closed densely defined operator $T$ in $\mathfrak{H}$ is affiliated with $\mathcal{A}\left(\mathcal{P}_{\mathcal{E}}\right)$ if and only if $T=J_{g}^{\mathcal{E}}$ for an $\mathcal{E}$-measurable complex function $\mathrm{g}$.

Proof. See Lemma 10 in Ref.4.

A family of maps $\left\{g_{\iota}\right\}_{\iota \in I}$ is said to separate points of a set $S$ if $S \subset D_{g_{\iota}}$ for all $\iota \in I$ and for any two distinct elements $s_{1}$ and $s_{2}$ of $S$, there is $\iota \in I$ such that $g_{\iota}\left(s_{1}\right) \neq g_{\iota}\left(s_{2}\right)$.

To cover both positive and spectral measures, the next definition is formulated in terms of a general A-valued measure (see Sec. A 2).

Definition III.5. Let $\mathfrak{A}$ be a topological Abelian group and $\nu$ be a $\sigma$-finite $\mathfrak{A}$-valued measure. A family $\left\{g_{\iota}\right\}_{\iota \in I}$ of maps is said to be $\nu$-separating if $I$ is countable and $\left\{g_{\iota}\right\}_{\iota \in I}$ separates points of $S_{\nu} \backslash N$ for some $\nu$-null set $N \stackrel{20}{?}$

The next result gives a complete description of systems of generators for $\mathcal{A}\left(\mathcal{P}_{\mathcal{E}}\right)$.

Proposition III.6. Let $\mathfrak{H}$ be a separable Hilbert space, $\mathcal{E}$ be a standard21 spectral measure in $\mathfrak{H}$, and $\mathfrak{X}$ be a set of closed densely defined operators in $\mathfrak{H}$. Then $\mathcal{A}(\mathfrak{X})=\mathcal{A}\left(\mathcal{P}_{\mathcal{E}}\right)$ if and only if the following conditions hold

1. $\mathcal{A}(\mathfrak{X}) \subset \mathcal{A}\left(\mathcal{P}_{\mathcal{E}}\right)$

2. There is an $\mathcal{E}$-separating family $\left\{g_{\iota}\right\}_{\iota \in I}$ of $\mathcal{E}$-measurable complex functions such that $J_{g_{\iota}}^{\mathcal{E}} \in \mathfrak{X}$ for all $\iota \in I$.

Proof. By Lemma III.2 and statement 2 of LemmaIII.4 condition 1 holds if and only if every element of $\mathfrak{X}$ is equal to $J_{g}^{\mathcal{E}}$ for some $\mathcal{E}$-measurable complex function $g$. Hence the proposition follows from Theorem 3 in Ref. 4.

Example III.7. Let $T$ be a normal ${ }^{22}$ operator in a separable Hilbert space and $\mathcal{E}_{T}$ be its spectral measure (see Sec. A 8). By Lemma II.2 and statement 2 of Lemma III.4, condition 1 of Proposition III.6 is fulfilled for every set $\mathfrak{X}$ whose elements are functions of $T$. Let $g$ be the identical function on $\mathbb{C}: g(z)=z, z \in \mathbb{C}$. Then the family containing the single function $g$ separates points of $\mathbb{C}$, and Proposition III.6 implies that the operator $T=g(T)$ is equivalent to the set $\mathcal{P}_{\mathcal{E}_{T}}$ of its spectral projections. Let $\zeta \in \mathbb{C}$ and $h_{\zeta}$ be the function on $\mathbb{C} \backslash\{\zeta\}$ defined by the relation $h_{\zeta}(z)=(z-\zeta)^{-1}$. If $\zeta$ is not an eigenvalue of $T$, then $\mathcal{E}_{T}(\{\zeta\})=0$ and the family containing the single function $h_{\zeta}$ is $\mathcal{E}_{T}$-separating. It follows from Proposition III.6 that the operator $(T-\zeta)^{-1}=h_{\zeta}(T)$ is equivalent to $\mathcal{P}_{\mathcal{E}_{T}}$ (and, hence, to $T$ ). Let $A \subset \mathbb{C}$ be a set having an accumulation point in $\mathbb{C}$ and let $f_{\zeta}(z)=e^{\zeta z}$ for $\zeta \in A$ and $z \in \mathbb{C}$. It is easy to show (see example 7 in Ref. 4 for details) that the family $\left\{f_{\zeta}\right\}_{\zeta \in A}$ contains a countable subfamily separating the points of $\mathbb{C}$. By Proposition $\amalg$ II.6, we conclude that the set of all operators $e^{\zeta T}$ with $\zeta \in A$ is equivalent to $\mathcal{P}_{\mathcal{E}_{T}}$.

\section{DIAGONALIZATIONS}

Given a positive $\sigma$-finite measure $\nu$, we say that a $\nu$-a.e. defined family $\mathfrak{S}$ of Hilbert spaces is $\nu$ nondegenerate if $\mathfrak{S}(s) \neq\{0\}$ for $\nu$-a.e. $s$.

Definition IV.1. Let $\mathfrak{X}$ be a set of closed densely defined operators in a Hilbert space $\mathfrak{H}$. A triple $(\nu, \mathfrak{S}, V)$, where $\nu$ is a positive $\sigma$-finite measure, $\mathfrak{S}$ is a $\nu$-nondegenerate $\nu$-measurable family of Hilbert spaces, and $V$ is a unitary operator from $\mathfrak{H}$ to $\int{ }^{\oplus} \mathfrak{S}(s) d \nu(s)$, is called a diagonalization for $\mathfrak{X}$ if every $T \in \mathfrak{X}$ is equal to $V^{-1} \mathcal{T}_{g}^{\nu, \mathfrak{S}} V$ for some complex $\nu$-measurable function $g$. A diagonalization $(\nu, \mathfrak{S}, V)$ for $\mathfrak{X}$ is called exact if condition (5) holds for any complex $\nu$-measurable $\nu$-essentially bounded function $g$. 
The next theorem gives an exactness criterion for diagonalizations, similar to condition (E) discussed in Introduction in the context of direct sum decompositions.

Theorem IV.2. Let $\mathfrak{H}$ be a separable Hilbert space and $\mathfrak{X}$ be a set of closed densely defined operators in $\mathfrak{H}$. A diagonalization $(\nu, \mathfrak{S}, V)$ for $\mathfrak{X}$, where $\nu$ is standard, is exact if and only if there is a $\nu$-separating family $\left\{g_{\iota}\right\}_{\iota \in I}$ of $\nu$-measurable complex functions such that

$$
V^{-1} \mathcal{T}_{g_{\iota}}^{\nu, \mathfrak{S}} V \in \mathfrak{X}, \quad \iota \in I .
$$

Sometimes the analysis of diagonalizations for $\mathfrak{X}$ simplifies if we replace $\mathfrak{X}$ with an equivalent set $\mathfrak{Y}$. The next result shows that passing to equivalent sets is always possible.

Proposition IV.3. Let $\mathfrak{H}$ be a separable Hilbert space and $\mathfrak{X}$ and $\mathfrak{Y}$ be equivalent sets of closed densely defined operators in $\mathfrak{H}$. Then every (exact) diagonalization for $\mathfrak{X}$ is an (exact) diagonalization for $\mathfrak{Y}$.

We say that normal operators $T_{1}$ and $T_{2}$ in $\mathfrak{H}$ commute if their spectral projections commute (if $T_{2} \in L(\mathfrak{H})$, then this definition agrees with Definition [.1, see Ref. 23). If a set $\mathfrak{X}$ of closed densely defined operators admits a diagonalization, then $\mathfrak{X}$ consists of normal pairwise commuting operators because operators of multiplication by functions are normal and commute with each other. The converse statement is provided by the next proposition.

Proposition IV.4. For every set of normal pairwise commuting operators in a separable Hilbert space, there exists a diagonalization $(\nu, \mathfrak{S}, V)$, where $\nu$ is standard.

Before proceeding with the proofs of the above results, we give some simple examples of diagonalizations.

Example IV.5. Let $Z$ be the set of all absolutely continuous square-integrable complex functions on $\mathbb{R}$ having square-integrable derivatives. Let $P$ be the one-dimensional operator of momentum, i.e., the operator in $L_{2}(\mathbb{R})$ with the domain $D_{P}=\left\{[f]_{\lambda}: f \in Z\right\}$ satisfying the relation

$$
\left(P[f]_{\lambda}\right)(x)=-i f^{\prime}(x), \quad f \in Z,
$$

for $\lambda$-a.e. $x$ (as in Sec. II $\lambda$ is the Lebesgue measure on $\mathbb{R}$ ). Let $\mathcal{F}: L_{2}(\mathbb{R}) \rightarrow L_{2}(\mathbb{R})$ be the operator of the Fourier transformation: $(\mathcal{F} f)(p)=(2 \pi)^{-1 / 2} \int f(x) e^{-i p x} d x$. Then we have $\mathcal{F} P \mathcal{F}^{-1}=\mathcal{T}_{g}^{\lambda}$, where $g$ is the identical function on $\mathbb{R}: g(p)=p, p \in \mathbb{R}$. By Theorem IV.2, we conclude that $\left(\lambda, \mathcal{I}_{\mathbb{C}, \lambda}, \mathcal{F}\right)$ is an exact diagonalization for $P$ (here, $\mathcal{I}_{\mathbb{C}, \lambda}$ is a constant family of Hilbert spaces, see Sec. [B3, by (B1), we have $\left.L_{2}(\mathbb{R})=\int^{\oplus} \mathcal{I}_{\mathbb{C}, \lambda}(x) d x\right)$. In view of Example III.7 and Proposition IV.3. $\left(\lambda, \mathcal{I}_{\mathbb{C}, \lambda}, \mathcal{F}\right)$ is also an exact diagonalization for the set $\left\{e^{i a P}\right\}_{a \in \mathbb{R}}$ of translations in $L_{2}(\mathbb{R})$.

Example IV.6. Let $P, \mathcal{F}$, and $g$ be as in Example IV.5 and let $H=P^{2}$ be the Hamiltonian of the onedimensional free particle. Then $\mathcal{F} H \mathcal{F}^{-1}=\mathcal{T}_{g^{2}}^{\lambda}$ and, hence, $\left(\lambda, \mathcal{I}_{\mathbb{C}, \lambda}, \mathcal{F}\right)$ is a diagonalization for $H$. Since the family containing the single function $g^{2}$ is not $\lambda$-separating, this diagonalization is not exact. Let $\mathbb{R}_{+}=$ $(0, \infty)$ and $V: L_{2}(\mathbb{R}) \rightarrow L_{2}\left(\mathbb{R}_{+}, \mathbb{C}^{2}, \lambda\right)$ be the unitary operator such that $(V f)(p)=((\mathcal{F} f)(p),(\mathcal{F} f)(-p))$, $f \in L_{2}(\mathbb{R})$, for $\lambda$-a.e. $p \in \mathbb{R}_{+}$. Then $V H V^{-1}$ is the operator of multiplication by $g^{2}$ in $L_{2}\left(\mathbb{R}_{+}, \mathbb{C}^{2}, \lambda\right)$. In view of (B1), it follows that $V H V^{-1}=\mathcal{T}_{g^{2}}^{\lambda_{+}, \mathfrak{S}}$, where $\lambda_{+}=\left.\lambda\right|_{\mathbb{R}_{+}}$and $\mathfrak{S}=\mathcal{I}_{\mathbb{C}^{2}, \lambda_{+}}$. Since $g^{2}$ separates points of $\mathbb{R}_{+}$, it follows from Theorem IV.2 that $\left(\lambda_{+}, \mathcal{I}_{\mathbb{C}^{2}, \lambda_{+}}, V\right)$ is an exact diagonalization for $H$.

We now turn to the proofs of Theorem IV.2 and Propositions IV.3 andIV.4. In the rest of this section, we assume that the Hilbert space $\mathfrak{H}$ is separable. For brevity, we say that $(\nu, \mathfrak{S}, V)$ is an $\mathfrak{H}$-triple if $\nu$ is a positive $\sigma$-finite measure, $\mathfrak{S}$ is a $\nu$-nondegenerate $\nu$-measurable family of Hilbert spaces, and $V$ is a unitary operator from $\mathfrak{H}$ to $\int^{\oplus} \mathfrak{S}(s) d \nu(s)$.

Given an $\mathfrak{H}$-triple $t=(\nu, \mathfrak{S}, V)$, we define the map $\mathcal{E}^{t}$ on $\sigma\left(D_{\nu}\right)$ by the relation

$$
\mathcal{E}^{t}(A)=V^{-1} \mathcal{T}_{\chi A}^{\nu, \mathfrak{S}} V, \quad A \in \sigma\left(D_{\nu}\right)
$$

where $\chi_{A}$ is equal to unity on $A$ and vanishes on $S_{\nu} \backslash A$.

Lemma IV.7. Let $t=(\nu, \mathfrak{S}, V)$ be an $\mathfrak{H}$-triple. Then $\mathcal{E}^{t}$ is a spectral measure such that $\mathcal{E}^{t}$-measurable and $\mathcal{E}^{t}$-null sets coincide with $\nu$-measurable and $\nu$-null sets respectively. For any $\nu$-measurable complex function $g$, we have $J_{g}^{\mathcal{E}^{t}}=V^{-1} \mathcal{T}_{g}^{\nu, \mathfrak{S}} V$. 
Proof. Clearly, $\mathcal{E}^{t}$ is an $L(\mathfrak{H})$-valued $\sigma$-additive function satisfying condition (a) of Sec. A 2 , Clearly, $\mathcal{N}_{\nu} \subset \mathcal{N}_{\mathcal{E}^{t}}$. If $N \in \mathcal{N}_{\mathcal{E}^{t}}$, then $\mathcal{T}_{\chi N}^{\nu, \mathfrak{S}}=0$ and, hence, $\nu(N)=0$ because $\mathfrak{S}(s) \neq 0$ for $\nu$-a.e. $s$. It follows that $\mathcal{N}_{\nu}=\mathcal{N}_{\mathcal{E}^{t}}$ and, therefore, condition (b) of Sec. A2 is also fulfilled. Thus, $\mathcal{E}^{t}$ is a spectral measure having the same null sets as $\nu$. Since $D_{\mathcal{E}^{t}}=\sigma\left(D_{\nu}\right)$, we have $\sigma\left(D_{\mathcal{E}^{t}}\right)=\sigma\left(D_{\nu}\right)$, i.e., $\nu$-measurable sets coincide with $\mathcal{E}^{t}$-measurable sets. For any $A \in D_{\mathcal{E}^{t}}$ and $\Psi \in \mathfrak{H}$, we have $\mathcal{E}_{\Psi}^{t}(A)=\int_{A}\|(V \Psi)(s)\|^{2} d \nu(s)$ (see Sec. A 8). Hence, a $\nu$-measurable function $g$ is $\mathcal{E}_{\Psi}^{t}$-integrable if and only if $s \rightarrow\|(V \Psi)(s)\|^{2} f(s)$ is a $\nu$-integrable function, in which case we have

$$
\int g(s) d \mathcal{E}_{\Psi}^{t}(s)=\int\|(V \Psi)(s)\|^{2} g(s) d \nu(s)
$$

Let $g$ be a $\nu$-measurable function and $\Psi \in \mathfrak{H}$. By (A1), we have

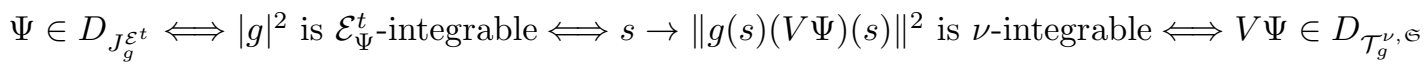

and, therefore, the domains of $J_{g}^{\mathcal{E}^{t}}$ and $V^{-1} \mathcal{T}_{g}^{\nu, \mathfrak{S}} V$ coincide. Now (A2) and (39) imply that

$$
\left\langle\Psi, J_{g}^{\mathcal{E}^{t}} \Psi\right\rangle=\int g(s) d \mathcal{E}_{\Psi}^{t}(s)=\left\langle V \Psi, \mathcal{T}_{g}^{\nu, \mathfrak{S}} V \Psi\right\rangle
$$

for any $\Psi \in D_{J_{g}^{\mathcal{E}}}$. Hence, $J_{g}^{\mathcal{E}^{t}}=V^{-1} \mathcal{T}_{g}^{\nu, \mathfrak{S}} V$.

Proposition IV.3 follows immediately from the next lemma.

Lemma IV.8. Let $\mathfrak{X}$ be a set of closed densely defined operators in $\mathfrak{H}$. An $\mathfrak{H}$-triple $t$ is an (exact) diagonalization for $\mathfrak{X}$ if and only if $\mathcal{A}(\mathfrak{X}) \subset \mathcal{A}\left(\mathcal{P}_{\mathcal{E}^{t}}\right)$ (resp., $\mathcal{A}(\mathfrak{X})=\mathcal{A}\left(\mathcal{P}_{\mathcal{E}^{t}}\right)$ ).

Proof. Let $t$ be an $\mathfrak{H}$-triple. Lemma IV.7 statement 2 of Lemma II.4, and Lemma II.2 imply that

$t$ is a diagonalization for $\mathfrak{X} \Longleftrightarrow$

$$
\begin{aligned}
\text { every element of } \mathfrak{X} \text { is equal to } J_{g}^{\mathcal{E}^{t}} \text { for some } \mathcal{E}^{t} \text {-measurable } g \Longleftrightarrow \\
\text { every element of } \mathfrak{X} \text { is affiliated with } \mathcal{A}\left(\mathcal{P}_{\mathcal{E}^{t}}\right) \Longleftrightarrow \mathcal{A}(\mathfrak{X}) \subset \mathcal{A}\left(\mathcal{P}_{\mathcal{E}^{t}}\right) .
\end{aligned}
$$

In view of statement 1 of Lemma III.4 an $\mathfrak{H}$-triple $t$ is an exact diagonalization for $\mathfrak{X}$ if and only if it is a diagonalization for $\mathfrak{X}$ and $\mathcal{A}(\mathfrak{X}) \supset \mathcal{A}\left(\mathcal{P}_{\mathcal{E}^{t}}\right)$. By the above, these two conditions are equivalent to the equality $\mathcal{A}(\mathfrak{X})=\mathcal{A}\left(\mathcal{P}_{\mathcal{E}^{t}}\right)$.

Proof of Theorem IV.2. Let $t=(\nu, \mathfrak{S}, V)$ be a diagonalization for $\mathfrak{X}$, where $\nu$ is standard. By Lemma IV.8 and Proposition III.6 $t$ is exact if and only if there is an $\mathcal{E}^{t}$-separating family $\left\{g_{\iota}\right\}_{\iota \in I}$ of $\mathcal{E}^{t}$-measurable complex functions such that $J_{g_{\iota}}^{\mathcal{E}^{t}} \in \mathfrak{X}$ for all $\iota \in I$. Hence, the required statement follows from Lemma IV.7.

Proof of Proposition IV.4. Let $\mathfrak{X}$ be a set of pairwise commuting normal operators in $\mathfrak{H}$ and $\mathfrak{Y}=$ $\bigcup_{T \in \mathfrak{X}} \mathcal{P}_{\mathcal{E}_{T}}$, where $\mathcal{E}_{T}$ is the spectral measure of $T$ (see Sec. A 8). Then $\mathfrak{Y}$ is an involutive subset of $L(\mathfrak{H})$ whose elements pairwise commute and, therefore, $\mathcal{M}=A(\mathfrak{Y})$ is an Abelian von Neumann algebra. Since $\mathcal{A}(T)=\mathcal{A}\left(\mathcal{P}_{\mathcal{E}_{T}}\right) \subset \mathcal{M}$ for any $T \in \mathfrak{X}$ (see Example III.7), every element of $\mathfrak{X}$ is affiliated with $\mathcal{M}$. It follows from Lemma III.2 that $\mathcal{A}(\mathfrak{X}) \subset \mathcal{M}$. Hence, the algebra $\mathcal{A}(\mathfrak{X})$ is Abelian. By Théorème 2 of Sec. II.6.2 in Ref. 19, there are a finite Borel measure $\nu$ on a compact metrizable space, a $\nu$-measurable family $\mathfrak{S}$ of Hilbert spaces, and a unitary operator $V: \mathfrak{H} \rightarrow \int^{\oplus} \mathfrak{S}(s) d \nu(s)$ such that $\mathcal{A}(\mathfrak{X})$ coincides with the set of all operators $V^{-1} \mathcal{T}_{g}^{\nu, \mathfrak{S}} V$, where $g$ is a $\nu$-measurable $\nu$-essentially bounded complex function. This means that $(\nu, \mathfrak{S}, V)$ is an exact diagonalization for $\mathcal{A}(\mathfrak{X})$. Since $\mathfrak{X}$ is equivalent to $\mathcal{A}(\mathfrak{X})$, Proposition IV.3 implies that $(\nu, \mathfrak{S}, V)$ is also an exact diagonalization for $\mathfrak{X}$.

\section{REDUCTION BY SYMMETRIES}

In this section, we assume that

$(A) \mathfrak{H}$ is a Hilbert space, $\mathfrak{X}$ is an involutive subset of $L(\mathfrak{H})$, and $(\nu, \mathfrak{S}, V)$ is an exact digonalization for $\mathfrak{X}$. 
Given a $\nu$-measurable family $\mathcal{H}$ of closed operators in $\mathfrak{S}$, we define the operator $Q_{\mathcal{H}}$ in $\mathfrak{H}$ by setting

$$
Q_{\mathcal{H}}=V^{-1} \int^{\oplus} \mathcal{H}(s) d \nu(s) V .
$$

The structure of closed operators in $\mathfrak{H}$ commuting with operators in $\mathfrak{X}$ and of their closed (in particular, self-adjoint) symmetry preserving extensions is described by the next theorem.

Theorem V.1. Let (A) be satisfied. Then the following statements hold:

1. $H$ is a closed operator in $\mathfrak{H}$ commuting with all elements of $\mathfrak{X}$ if and only if $H=Q_{\mathcal{H}}$ for some $\nu$-measurable family $\mathcal{H}$ of closed operators in $\mathfrak{S}$.

2. Let $\mathcal{H}$ and $\tilde{\mathcal{H}}$ be $\nu$-measurable families of closed operators in $\mathfrak{S}$. Then $Q_{\tilde{\mathcal{H}}}$ is an extension of $Q_{\mathcal{H}}$ if and only if $\tilde{\mathcal{H}}(s)$ is an extension of $\mathcal{H}(s)$ for $\nu$-a.e. $s$. In particular, $Q_{\tilde{\mathcal{H}}}=Q_{\mathcal{H}}$ if and only if $\tilde{\mathcal{H}}(s)=\mathcal{H}(s)$ for $\nu$-a.e. $s$.

3. Let $\mathcal{H}$ be a $\nu$-measurable family of closed operators in $\mathfrak{S}$. Then $Q_{\mathcal{H}}$ is self-adjoint if and only if $\mathcal{H}(s)$ is self-adjoint for $\nu$-a.e. $s$.

Proof. Let $\mathcal{H}$ be a $\nu$-measurable family of closed operators in $\mathfrak{S}$. Since $\mathfrak{X} \subset L(\mathfrak{H})$, every element of $\mathfrak{X}$ is representable in the form $V^{-1} \mathcal{T}_{g}^{\nu, \mathfrak{S}} V$, where $g$ is $\nu$-essentially bounded. By Proposition $\mathbb{B} .22$, $\int^{\oplus} \mathcal{H}(s) d \nu(s)$ is a closed operator commuting with all $\mathcal{T}_{g}^{\nu, \mathfrak{S}}$ and, therefore, $Q_{\mathcal{H}}$ is a closed operator commuting with all elements of $\mathfrak{X}$. Conversely, let $H$ be a closed operator in $\mathfrak{H}$ commuting with all elements of $\mathfrak{X}$. Let $\mathcal{M}$ denote the subalgebra of $L(\mathfrak{H})$ consisting of all operators commuting with $H$. By Lemma 1 in Ref. $4, \mathcal{M}$ is strongly closed. Since $\mathfrak{X}$ is involutive, $\mathcal{A}(\mathfrak{X})$ coincides with the smallest strongly closed subalgebra of $L(\mathfrak{H})$ containing $\mathfrak{X}$ and the identity operator in $\mathfrak{H}$. We hence have $\mathcal{A}(\mathfrak{X}) \subset \mathcal{M}$, i.e., $H$ commutes with all operators in $\mathcal{A}(\mathfrak{X})$. As the diagonalization $(\nu, \mathfrak{S}, V)$ is exact, it follows from (5) that $V H V^{-1}$ commutes with all operators $\mathcal{T}_{g}^{\nu, \mathfrak{S}}$, where $g$ is a $\nu$-measurable $\nu$-essentially bounded function. By Proposition B.22, we have $V H V^{-1}=\int^{\oplus} \mathcal{H}(s) d \nu(s)$ for some $\nu$-measurable family $\mathcal{H}$ of closed operators in $\mathfrak{S}$. This means that $H=Q_{\mathcal{H}}$ and statement 1 is proved. Statements 2 and 3 follow immediately from Proposition B.21 and Corollary B.24 respectively.

In particular, Statements 1 and 2 of Theorem V.1 imply the existence and uniqueness (up to $\nu$ equivalence) of decomposition (4) for any closed operator $H$ commuting with all elements of $\mathfrak{X}$.

Corollary V.2. Let (A) be satisfied and $\mathcal{H}$ be a $\nu$-measurable family of closed operators in $\mathfrak{S}$. Then the closed (resp., self-adjoint) extensions of $Q_{\mathcal{H}}$ commuting with all elements of $\mathfrak{X}$ are precisely the operators $Q_{\tilde{\mathcal{H}}}$, where $\tilde{\mathcal{H}}$ is a $\nu$-measurable family of operators in $\mathfrak{S}$ such that $\tilde{\mathcal{H}}(s)$ is a closed (resp., self-adjoint) extension of $\mathcal{H}(s)$ for $\nu$-a.e. $s$.

In concrete examples, $H$ usually comes as the closure of some non-closed operator $\check{H}$. It is often possible to establish by a direct computation that $Q_{\mathcal{H}}$ is an extension of $\check{H}$ for some $\nu$-a.e. defined family $\mathcal{H}$ of closed operators. In this case, the next proposition may be used to prove that actually equality (4) holds.

Proposition V.3. Let (A) be satisfied, $\check{H}$ be an operator in $\mathfrak{H}$, and $\mathcal{H}$ be a $\nu$-a.e. defined family of closed operators in $\mathfrak{S}$ such that $Q_{\mathcal{H}}$ is an extension of $\tilde{H}$. Suppose $D_{\breve{H}}$ is taken to itself by all operators in $\mathfrak{X}$ and there is a sequence $\xi_{1}, \xi_{2}, \ldots$ of elements of $V\left(D_{\breve{H}}\right)$ such that the linear span of $\left(\xi_{j}(s), \mathcal{H}(s) \xi_{j}(s)\right)$ is dense in the graph $G_{\mathcal{H}(s)}$ of $\mathcal{H}(s)$ for $\nu$-a.e. s. Then $\mathcal{H}$ is $\nu$-measurable, $\check{H}$ is closable, and $\overline{\check{H}}=Q_{\mathcal{H}}$.

Proof. Since $\xi_{j} \in D_{V \check{H} V^{-1}}$ for all $j=1,2, \ldots$ and $\int^{\oplus} \mathcal{H}(s) d \nu(s)$ is an extension of $V \check{H} V^{-1}$, we have $\mathcal{H}(s) \xi_{j}(s)=\left(V \check{H} V^{-1} \xi_{j}\right)(s)$ for $\nu$-a.e. $s$ and, hence, $s \rightarrow \mathcal{H}(s) \xi_{j}(s)$ are $\nu$-measurable sections of $\mathfrak{S}$. It follows that the family $\mathcal{H}$ is $\nu$-measurable. Statement 1 of Theorem V.1 implies that $Q_{\mathcal{H}}$ is a closed operator commuting with all elements of $\mathfrak{X}$. Since $Q_{\mathcal{H}}$ is an extension of $H_{\text {and }} D_{\check{H}}$ is taken to itself by all operators in $\mathfrak{X}$, it follows that $\check{H}$ is closable and commutes with all elements of $\mathfrak{X}$. By Lemma III.1. we conclude that $H=\bar{H}$ also commutes with all operators in $\mathfrak{X}$. By statement 1 of Theorem V.1 there is a $\nu$-measurable family $\mathcal{H}_{0}$ of closed operators in $\mathfrak{S}$ such that $H=Q_{\mathcal{H}_{0}}$. As $Q_{\mathcal{H}}$ is a closed extension of $\check{H}$, it is also an extension of $H$. By statement 2 of Theorem V.1. it follows that $\mathcal{H}(s)$ is an extension of $\mathcal{H}_{0}(s)$ for $\nu$-a.e. $s$. Since $V\left(D_{\check{H}}\right) \subset V\left(D_{H}\right)$ and $V\left(D_{H}\right)$ coincides with the domain of $\int^{\oplus} \mathcal{H}_{0}(s) d \nu(s)$, we have $\xi_{j}(s) \in D_{\mathcal{H}_{0}(s)}$ and $\mathcal{H}_{0}(s) \xi_{j}(s)=\mathcal{H}(s) \xi_{j}(s)$ for all $j$ and $\nu$-a.e. $s$. Thus, the linear span of $\left(\xi_{j}(s), \mathcal{H}(s) \xi_{j}(s)\right)$ is contained in $G_{\mathcal{H}_{0}(s)}$ and, hence, $G_{\mathcal{H}_{0}(s)}$ is dense in $G_{\mathcal{H}(s)}$ for $\nu$-a.e. $s$. In view of the closedness of $\mathcal{H}_{0}(s)$, this implies that $\mathcal{H}_{0}(s)=\mathcal{H}(s)$ for $\nu$-a.e. $s$. 


\section{ONE-DIMENSIONAL SCHRÖDINGER OPERATORS}

In this section, we recall basic facts (see, e.g., Refs. 24 26) concerning one-dimensional Schrödinger operators and then briefly discuss self-adjoint realizations of differential expression (22) and their eigenfunction expansions. In Sec. VIII, we shall use the results of Sec. V] to represent self-adjoint extensions of the Aharonov-Bohm Hamiltonian as direct integrals of one-dimensional Schrödinger operators of type (22). The proof of Theorem II.5 given in Sec. $\mathrm{X}$ is based on combining such a representation with the analysis of the one-dimensional problem given in this section.

Let $-\infty \leq a<b \leq \infty$ and $\lambda_{a, b}$ be the restriction to $(a, b)$ of the Lebesgue measure $\lambda$ on $\mathbb{R}$. We denote by $\mathcal{D}$ the space of all complex continuously differentiable functions on $(a, b)$ whose derivative is absolutely continuous on $(a, b)$ (i.e., absolutely continuous on every segment $[c, d]$ with $a<c \leq d<b$ ). Given a locally integrable real function $q$ on $(a, b)$, we denote by $l_{q}$ the linear operator from $\mathcal{D}$ to the space of complex $\lambda_{a, b}$-equivalence classes such that

$$
l_{q} f(r)=-f^{\prime \prime}(r)+q(r) f(r)
$$

for $\lambda$-a.e. $r \in(a, b)$. For every $c \in(a, b)$ and complex numbers $z_{1}$ and $z_{2}$, there is a unique solution $f$ of the equation $l_{q} f=0$ such that $f(c)=z_{1}$ and $f^{\prime}(c)=z_{2}$. This implies that solutions of $l_{q} f=0$ constitute a two-dimensional subspace of $\mathcal{D}$. For any functions $f, g \in \mathcal{D}$, their Wronskian $W_{r}(f, g)$ at point $r \in(a, b)$ is defined by the relation

$$
W_{r}(f, g)=f(r) g^{\prime}(r)-f^{\prime}(r) g(r) .
$$

Clearly, $r \rightarrow W_{r}(f, g)$ is an absolutely continuous function on $(a, b)$. If $f$ and $g$ are such that $r \rightarrow W_{r}(f, g)$ is a constant function on $(a, b)$ (this is the case, in particular, when $f$ and $g$ are solutions of $l_{q} f=l_{q} g=0$ ), its value will be denoted by $W(f, g)$. Let

$$
\mathcal{D}_{q}=\left\{f \in \mathcal{D}: f \text { and } l_{q} f \text { are both square-integrable on }(a, b)\right\} .
$$

A $\lambda_{a, b}$-measurable complex function $f$ is said to be left (right) square-integrable on $(a, b)$ if $\int_{a}^{c}|f(r)|^{2} d r<$ $\infty$ (resp., $\int_{c}^{b}|f(r)|^{2} d x<\infty$ ) for any $c \in(a, b)$. The subspace of $\mathcal{D}$ consisting of left (right) squareintegrable on $(a, b)$ functions $f$ such that $l_{q} f$ is also left (resp., right) square-integrable on $(a, b)$ will be denoted by $\mathcal{D}_{q}^{l}$ (resp., $\mathcal{D}_{q}^{r}$ ). We obviously have $\mathcal{D}_{q}=\mathcal{D}_{q}^{l} \cap \mathcal{D}_{q}^{r}$. It follows from (40) by integrating by parts that

$$
\int_{c}^{d}\left(\left(l_{q} f\right)(r) g(r)-f(r)\left(l_{q} g\right)(r)\right) d r=W_{d}(f, g)-W_{c}(f, g)
$$

for every $f, g \in \mathcal{D}$ and $c, d \in(a, b)$. This implies the existence of limits $W_{a}(f, g)=\lim _{r \downarrow a} W_{r}(f, g)$ and $W_{b}(f, g)=\lim _{r \uparrow b} W_{r}(f, g)$ for $f, g \in \mathcal{D}_{q}^{l}$ and $f, g \in \mathcal{D}_{q}^{r}$ respectively. Moreover, it follows that ${ }^{27}$

$$
\left\langle l_{q} f,[g]\right\rangle-\left\langle[f], l_{q} g\right\rangle=W_{b}(\bar{f}, g)-W_{a}(\bar{f}, g)
$$

for any $f, g \in \mathcal{D}_{q}$, where $\langle\cdot, \cdot\rangle$ is the scalar product in $L_{2}(a, b)$.

For any linear subspace $Z$ of $\mathcal{D}_{q}$, let $L_{q}(Z)$ be the linear operator in $L_{2}(a, b)$ defined by the relations

$$
\begin{aligned}
& D_{L_{q}(Z)}=\{[f]: f \in Z\}, \\
& L_{q}(Z)[f]=l_{q} f, \quad f \in Z .
\end{aligned}
$$

We define the minimal operator $L_{q}$ by setting

$$
L_{q}=L_{q}\left(\mathcal{D}_{q}^{0}\right)
$$

where

$$
\mathcal{D}_{q}^{0}=\left\{f \in \mathcal{D}_{q}: W_{a}(f, g)=W_{b}(f, g)=0 \text { for any } g \in \mathcal{D}_{q}\right\}
$$

By (42), the operator $L_{q}(Z)$ is symmetric if and only if $W_{a}(\bar{f}, g)=W_{b}(\bar{f}, g)$ for any $f, g \in Z$. In particular, $L_{q}$ is a symmetric operator. Moreover, $L_{q}$ is closed and densely defined and its adjoint $L_{q}^{*}$ is given by

$$
L_{q}^{*}=L_{q}\left(\mathcal{D}_{q}\right)
$$


(see Ref. 25, Lemma 9.4). If $T$ is a symmetric extension of $L_{q}$, then $L_{q}^{*}$ is an extension of $T^{*}$ and, hence, of $T$. In view of (45), this implies that $T$ is of the form $L_{q}(Z)$ for some subspace $Z$ of $\mathcal{D}_{q}$.

If $W_{a}(f, g)=0$ for any $f, g \in \mathcal{D}_{q}^{l}$, then $q$ is said to be in the limit point case (l.p.c.) at $a$. Otherwise $q$ is said to be in the limit circle case (l.c.c.) at $a$. Similarly, $q$ is said to be in the l.p.c. at $b$ if $W_{b}(f, g)=0$ for any $f, g \in \mathcal{D}_{q}^{r}$ and to be in l.c.c. at $b$ otherwise. According to the well-known Weyl alternative (see, e.g., Ref 25, Theorem 9.9), $q$ is in l.c.c. at $a$ if and only if all solutions of $l_{q} f=0$ are left square-integrable on $(a, b)$ (and, hence, belong to $\mathcal{D}_{q}^{l}$ ).

If $q$ is in l.p.c. at both $a$ and $b$, then (45) implies that $L_{q}^{*}$ is symmetric and, therefore, $L_{q}$ is self-adjoint.

Suppose now that $q$ is in l.c.c. at $a$. Let $f_{1}, f_{2} \in \mathcal{D}$ be linearly independent functions such that $l_{q} f_{1}=l_{q} f_{2}=0$. Then $f_{1}$ and $f_{2}$ are left square-integrable on $(a, b)$ and, therefore, the function

$$
\delta_{q}^{g}(r)=\frac{1}{W\left(f_{1}, f_{2}\right)}\left[f_{1}(r) \int_{a}^{r}\left(l_{q} g\right)(\rho) f_{2}(\rho) d \rho-f_{2}(r) \int_{a}^{r}\left(l_{q} g\right)(\rho) f_{1}(\rho) d \rho\right]
$$

is well-defined and belongs to $\mathcal{D}$ for any $g \in \mathcal{D}_{q}$. It is straightforward to check that $\delta_{q}^{g}$ does not depend on the choice of the solutions $f_{1}$ and $f_{2}$ and $l_{q} \delta_{q}^{g}=l_{q} g$. Hence, the function

$$
\gamma_{q}^{g}=g-\delta_{q}^{g}
$$

satisfies the equation

$$
l_{q} \gamma_{q}^{g}=0 .
$$

Let $q$ be in l.c.c. at $a$ and in l.p.c. at $b$. In this case, $L_{q}$ has deficiency indices $(1,1)$ and the self-adjoint extensions of $L_{q}$ are precisely the operators (see Ref. 18, Lemma 11)

$$
L_{q}^{f}=L_{q}\left(Z_{q}^{f}\right)
$$

where $f$ is a nontrivial real solution of $l_{q} f=0$ and the subspace $Z_{q}^{f}$ of $\mathcal{D}_{q}$ is given by

$$
Z_{q}^{f}=\left\{g \in \mathcal{D}_{q}: W_{a}(f, g)=0\right\}
$$

The operator $L_{q}^{f}$ determines $f$ uniquely up to a nonzero real coefficient. Since the deficiency indices of $L_{q}$ are both equal to 1 , the orthogonal complement $G_{L_{q}^{f}} \ominus G_{L_{q}}$ of the graph $G_{L_{q}}$ of $L_{q}$ in the graph $G_{L_{q}^{f}}$ of $L_{q}^{f}$ is one-dimensional.

Lemma VI.1. Suppose $q$ is in l.c.c. at a and in l.p.c. at b. Let $T$ be a self-adjoint extension of $L_{q}$ and $g$ be a real function in $\mathcal{D}_{q}$ such that $[g] \in D_{T} \backslash D_{L_{q}}$. Then $\gamma_{q}^{g}$ is a real nontrivial solution of (48) and $T=L_{q}^{\gamma_{q}^{g}}$.

Proof. The reality of $\gamma_{q}^{g}$ follows from (46) and (47) because we can choose $f_{1}$ and $f_{2}$ in (46) to be real. It follows easily from (46) that

$$
W_{r}\left(\delta_{q}^{g}, h\right)=\frac{1}{W\left(f_{1}, f_{2}\right)}\left[W_{r}\left(f_{1}, h\right) \int_{a}^{r}\left(l_{q} g\right)(\rho) f_{2}(\rho) d \rho-W_{r}\left(f_{2}, h\right) \int_{a}^{r}\left(l_{q} g\right)(\rho) f_{1}(\rho) d \rho\right], \quad r \in(a, b),
$$

for any $h \in \mathcal{D}$, where $f_{1}, f_{2}$ are linearly independent solutions of $l_{q} f_{1,2}=0$. This implies that

$$
W_{a}\left(\delta_{q}^{g}, h\right)=0
$$

for any $h \in \mathcal{D}_{q}^{l}$ and, therefore, $W_{a}(g, h)=W_{a}\left(\gamma_{q}^{g}, h\right)$. If $\gamma_{q}^{g}$ were trivial, we would have $W_{a}(g, h)=$ $W_{b}(g, h)=0$ for any $h \in \mathcal{D}_{q}$ (recall that $q$ is in l.p.c. at $b$ ) and, hence, $[g] \in D_{L_{q}}$ by (44) and (43). Thus, $\gamma_{q}^{g}$ is nontrivial. Let $f$ be a nontrivial real solution of $l_{q} f=0$ such that $T=L_{q}^{f}$. Then we have $W_{a}(f, g)=0$, and it follows from (47) and (51) that $W\left(f, \gamma_{q}^{g}\right)=0$. This means that $\gamma_{q}^{g}=C f$ for some real $C \neq 0$ and, therefore, $T=L_{q}^{\gamma_{q}^{g}}$.

If $q$ is locally square-integrable on $(a, b)$, then $\mathcal{D}_{q}^{0} \supset C_{0}^{\infty}(a, b)$, where $C_{0}^{\infty}(a, b)$ is the space of smooth functions on $(a, b)$ with compact support. In view of (43), this implies that $\left([f], l_{q} f\right) \in G_{L_{q}}$ for any $f \in C_{0}^{\infty}(a, b)$.

Lemma VI.2. There exists a countable set $A \subset C_{0}^{\infty}(a, b)$ such that the elements $\left([f], l_{q} f\right)$ with $f \in A$ are dense in the graph $G_{L_{q}}$ of $L_{q}$ for any locally square-integrable real function $q$ on $(a, b)$. 
Proof. Given $a<\alpha \leq \beta<b$, we denote by $Y_{\alpha, \beta}$ the linear subspace of the space $C_{0}^{\infty}(a, b)$ consisting of all its elements vanishing outside of $[\alpha, \beta]$. We make $Y_{\alpha, \beta}$ a normed space by setting

$$
\|f\|=\sup _{\alpha \leq r \leq \beta}\left(|f(r)|+\left|f^{\prime \prime}(r)\right|\right), \quad f \in Y_{\alpha, \beta} .
$$

Let $C[\alpha, \beta]$ be the space of all continuous functions on the segment $[\alpha, \beta]$. Then $f \rightarrow\left(f, f^{\prime \prime}\right)$ is an isometric embedding of $Y_{\alpha, \beta}$ into $C[\alpha, \beta]^{2}$ if the latter space is endowed with the norm

$$
\|(f, g)\|=\sup _{\alpha \leq r \leq \beta}(|f(r)|+|g(r)|), \quad f, g \in C[\alpha, \beta] .
$$

Note that the space $C[\alpha, \beta]^{2}$ with this norm is separable because $C[\alpha, \beta]$ endowed with its ordinary supremum norm is separable. Since every subspace of a separable metric space is separable, it follows that $Y_{\alpha, \beta}$ is separable. We now pick sequences $\alpha_{1}, \alpha_{2}, \ldots$ and $\beta_{1}, \beta_{2}, \ldots$ such that $a<\alpha_{j} \leq \beta_{j}<b$ for all $j$ and $\alpha_{j} \rightarrow a$ and $\beta_{j} \rightarrow b$ as $j \rightarrow \infty$. For each $j=1,2, \ldots$, we choose a countable dense subset $A_{j}$ of $Y_{\alpha_{j}, \beta_{j}}$ and define the set $A$ by the relation $A=\bigcup_{j=1}^{\infty} A_{j}$. If $q$ is a locally square-integrable real function on $(a, b)$, then $L_{q}$ is the closure of $L_{q}\left(C_{0}^{\infty}(a, b)\right)$ by Lemma 17 of Ref. 18. This means that elements of the form $\left([f], l_{q} f\right)$ with $f \in C_{0}^{\infty}(a, b)$ are dense in the graph of $L_{q}$, and it suffices to prove that every such element can be approximated by $\left([g], l_{q} g\right)$ with $g \in A$. For this, we choose a $j$ such that $f \in Y_{\alpha_{j}, \beta_{j}}$ and find a sequence $g_{1}, g_{2}, \ldots$ of elements of $A_{j}$ converging to $f$ in $Y_{\alpha_{j}, \beta_{j}}$. As $l_{q}$ obviously induces a continuous map from $Y_{\alpha_{j}, \beta_{j}}$ to $L_{2}(a, b)$ and $f \rightarrow[f]$ is a continuous embedding of $Y_{\alpha_{j}, \beta_{j}}$ into $L_{2}(a, b)$, we conclude that $\left[g_{k}\right] \rightarrow[f]$ and $l_{q} g_{k} \rightarrow l_{q} f$ in $L_{2}(a, b)$ as $k \rightarrow \infty$.

We now turn to the Schrödinger operators corresponding to differential expression (22) that arises as a result of the separation of variables in the Aharonov-Bohm model. We therefore assume $a=0$ and $b=\infty$ and define the potential $q_{\kappa}$ on $\mathbb{R}_{+}$by the relation

$$
q_{\kappa}(r)=\frac{\kappa^{2}-1 / 4}{r^{2}}
$$

for every $\kappa \in \mathbb{R}$. Let

$$
h_{\kappa}=L_{q_{\kappa}} .
$$

Our aim is to describe all self-adjoint extensions of $h_{\kappa}$ and their eigenfunction expansions.

The equation $l_{q_{\kappa}} f=0$ has linearly independent solutions $r^{1 / 2 \pm \kappa}$ for $\kappa \neq 0$ and $r^{1 / 2}$ and $r^{1 / 2} \ln r$ for $\kappa=0$. This implies that

(i) $q_{\kappa}$ is in l.p.c. at both 0 and $\infty$ for $|\kappa| \geq 1$,

(ii) $q_{\kappa}$ is in l.p.c. at $\infty$ and in l.c.c. at 0 for $|\kappa|<1$.

For every $E \in \mathbb{R}$, let the function $u^{\kappa}(E)$ on $\mathbb{R}_{+}$be given by (13). By (21), $u^{\kappa}(E)$ are generalized eigenfunctions for differential expression (22). In particular, for $E=0$, we have

$$
l_{q_{\kappa}} u^{\kappa}(0)=0
$$

Let $L_{2}^{c}\left(\mathbb{R}_{+}\right)$denote the subspace of $L_{2}\left(\mathbb{R}_{+}\right)$consisting of all its elements vanishing $\lambda$-a.e. outside some compact subset of $\mathbb{R}_{+}$.

If $|\kappa| \geq 1$, then the operator $h_{\kappa}$ is self-adjoint by (i). It is well known $24,28-30$ that this operator can be diagonalized by the Hankel transformation. In terms of the functions $u^{\kappa}(E)$, this result can be formulated as follows (see Theorem 1 and formula (36) in Ref. 18).

Proposition VI.3. Let $|\kappa| \geq 1$ and the positive measure $\mathcal{V}_{\kappa}$ on $\mathbb{R}$ be defined by (23). Then there is a unique unitary operator $U_{\kappa}: L_{2}\left(\mathbb{R}_{+}\right) \rightarrow L_{2}\left(\mathbb{R}, \mathcal{V}_{\kappa}\right)$ such that

$$
\left(U_{\kappa} \psi\right)(E)=\int_{0}^{\infty} u^{|\kappa|}(E \mid r) \psi(r) d r, \quad \psi \in L_{2}^{c}\left(\mathbb{R}_{+}\right),
$$

for $\mathcal{V}_{\kappa}$-a.e. E. We have $U_{\kappa} h_{\kappa} U_{\kappa}^{-1}=\mathcal{T}_{\iota}^{\mathcal{V}_{\kappa}}$, where $\iota$ is the identity function on $\mathbb{R}$ (i.e., $\iota(E)=E$ for all $E \in \mathbb{R})$. 
Suppose now that $-1<\kappa<1$. For every $\vartheta, E \in \mathbb{R}$, let the function $u_{\vartheta}^{\kappa}(E)$ on $\mathbb{R}_{+}$be defined by (15) and (16). As was shown in Sec. III. equality (21) remains valid for $u_{\vartheta}^{\kappa}(E)$ in place of $u^{ \pm \kappa}(E)$ and, hence, $u_{\vartheta}^{\kappa}(E)$ are generalized eigenfunctions for differential expression (22). For $E=0$, this means that

$$
l_{q_{\kappa}} u_{\vartheta}^{\kappa}(0)=0
$$

It follows immediately from (15) and (16) that

$$
u_{\vartheta}^{\kappa}(E)=u^{\kappa}(E) \cos \left(\vartheta-\vartheta_{\kappa}\right)+w^{\kappa}(E) \sin \left(\vartheta-\vartheta_{\kappa}\right)
$$

for all $\vartheta, E \in \mathbb{R}$, where $\vartheta_{\kappa}$ is defined by (17) and

$$
w^{\kappa}(E)=u_{\pi / 2+\vartheta_{\kappa}}^{\kappa}(E), \quad E \in \mathbb{R} .
$$

By (56) and (58), we have

$$
l_{q_{\kappa}} w^{\kappa}(0)=0 \text {. }
$$

It follows immediately from (13), (15), (16), and (58) that

$$
W\left(u^{\kappa}(0), w^{\kappa}(0)\right)=\frac{2}{\pi}
$$

and, therefore, $u^{\kappa}(0)$ and $w^{\kappa}(0)$ are real linearly independent elements of $\mathcal{D}$ for every $-1<\kappa<1$. By (54), (56), (57), and (59), we conclude that the set of all real nontrivial $f \in \mathcal{D}$ such that $l_{q_{\kappa}} f=0$ coincides with the set of all elements of the form $c u_{\vartheta}^{\kappa}(0)$, where $c, \vartheta \in \mathbb{R}$ and $c \neq 0$. In view of (53) and (ii), this implies that the self-adjoint extensions of $h_{\kappa}$ are precisely the operators

$$
h_{\kappa, \vartheta}=L_{q_{\kappa}}^{u_{\vartheta}^{\kappa}(0)}
$$

where $\vartheta \in \mathbb{R}$. Given $\vartheta \in \mathbb{R}$, let the positive measure $\mathcal{V}_{\kappa, \vartheta}$ on $\mathbb{R}$ be defined by (24) and (27) for $0<|\kappa|<1$ and $\kappa=0$ respectively. The next proposition gives eigenfunction expansions for $h_{\kappa, \vartheta}$.

Proposition VI.4. For every $-1<\kappa<1$ and $\vartheta \in \mathbb{R}$, there is a unique unitary operator $U_{\kappa, \vartheta}: L_{2}\left(\mathbb{R}_{+}\right) \rightarrow$ $L_{2}\left(\mathbb{R}, \mathcal{V}_{\kappa, \vartheta}\right)$ such that

$$
\left(U_{\kappa, \vartheta} \psi\right)(E)=\int_{0}^{\infty} u_{\vartheta}^{\kappa}(E \mid r) \psi(r) d r, \quad \psi \in L_{2}^{c}\left(\mathbb{R}_{+}\right)
$$

for $\mathcal{V}_{\kappa, \vartheta}$-a.e. E. We have $U_{\kappa, \vartheta} h_{\kappa, \vartheta} U_{\kappa, \vartheta}^{-1}=\mathcal{T}_{\iota}^{\mathcal{V}_{\kappa, \vartheta}}$, where $\iota$ is the identity function on $\mathbb{R}$.

Proof. See Theorem 3 and formulas (36) and (57) in Ref. 18.

Remark VI.5. Another treatment of self-adjoint realizations of (22) and their eigenfunction expansions can be found in Ref. 29. Our consideration differs from that in Ref. 29 by the choice of parametrization of self-adjoint extensions. The advantage of our choice is that the generalized eigenfunctions $u_{\vartheta}^{\kappa}(E \mid r)$ and the measures $\mathcal{V}_{\kappa, \vartheta}$ are continuous in $\kappa$ at $\kappa=0$.

\section{MEASURABLE FAMILIES OF ONE-DIMENSIONAL SCHRÖDINGER OPERATORS}

In this section, we derive a criterion for the measurability of families of one-dimensional Schrödinger operators. In Sec. VIII, we shall use this criterion together with Corollary V.2 to obtain direct integral representations for self-adjoint extensions of $H^{\phi}$ commuting with $T_{G}$ for all $G \in \mathcal{G}$.

In what follows, we fix $-\infty \leq a<b \leq \infty$ and set $\mathfrak{h}=L_{2}(a, b)$.

Let $\nu$ be a $\sigma$-finite positive measure. A $\nu$-a.e. defined map $\xi$ is said to be a $\nu$-measurable family of functions on $(a, b)$ if $\xi(s)$ is a locally integrable complex function on $(a, b)$ for $\nu$-a.e. $s$ and $s \rightarrow \int_{\alpha}^{\beta} \xi(s \mid r) d r$ is a $\nu$-measurable complex function for any $a<\alpha \leq \beta<b$.

Lemma VII.1. Let $\nu$ be a $\sigma$-finite positive measure. Then the following statements hold:

1. Let $\xi$ and $\eta$ be $\nu$-measurable families of locally square-integrable functions on $(a, b)$. Then $s \rightarrow$ $\xi(s) \eta(s)$ is a $\nu$-measurable family of functions on $(a, b)$. 
2. $\xi$ is an $\mathfrak{h}$-valued $\nu$-measurable map if and only if $\xi$ is a $\nu$-measurable family of functions on $(a, b)$ such that $\xi(s) \in \mathfrak{h}$ for $\nu$-a.e. $s$.

3. Let $\xi$ be a $\nu$-a.e. defined map such that $\xi(s)$ is a continuous function on $(a, b)$ for $\nu$-a.e. s. Then $\xi$ is a $\nu$-measurable family of functions on $(a, b)$ if and only if $s \rightarrow \xi(s \mid r)$ is a $\nu$-measurable complex function for any $r \in(a, b)$.

4. Let $r_{0} \in(a, b), \xi$ be a $\nu$-measurable family of functions on $(a, b)$, and $\eta$ be a $\nu$-a.e. defined map such that, for $\nu$-a.e. $s, \eta(s)$ is a complex function on $(a, b)$ satisfying the equality $\eta(s \mid r)=\int_{r_{0}}^{r} \xi(s \mid r) d r$ for all $r \in(a, b)$. Then $\eta$ is a $\nu$-measurable family of functions on $(a, b)$.

5. Let $\xi$ be a $\nu$-measurable family of functions on $(a, b)$ such that $\xi(s)$ is absolutely continuous on $(a, b)$ for $\nu$-a.e. $s$. Then $s \rightarrow \xi(s)^{\prime}$ is a $\nu$-measurable family of functions on $(a, b)$.

Proof. 1. For any $a<\gamma \leq \delta<b$, let $\chi_{\gamma, \delta}$ be the function on $(a, b)$ that is equal to unity on $[\gamma, \delta]$ and vanishes outside this segment. Then the linear span of all $\left[\chi_{\gamma, \delta}\right]$ with rational $\gamma$ and $\delta$ is dense in $\mathfrak{h}$. Let $e_{1}, e_{2}, \ldots$ be a basis in $\mathfrak{h}$ obtained by orthogonalization of this system. Given $a<\alpha \leq \beta<b$, we have $e^{31}$

$$
\begin{aligned}
\int_{\alpha}^{\beta} \xi(s \mid r) \eta(s \mid r) d r=\left\langle\left[\chi_{\alpha, \beta} \overline{\xi(s)}\right],\left[\chi_{\alpha, \beta} \eta(s)\right]\right\rangle= \\
=\sum_{i=1}^{\infty}\left\langle\left[\chi_{\alpha, \beta} \overline{\xi(s)}\right], e_{i}\right\rangle\left\langle e_{i},\left[\chi_{\alpha, \beta} \eta(s)\right]\right\rangle=\sum_{i=1}^{\infty} \int_{\alpha}^{\beta} \xi(s \mid r) e_{i}(r) d r \int_{\alpha}^{\beta} e_{i}(r) \eta(s \mid r) d r
\end{aligned}
$$

for $\nu$-a.e. $s$, where $\langle\cdot, \cdot\rangle$ is the scalar product in $\mathfrak{h}$. Since each $e_{i}$ is $\lambda_{a, b}$-equivalent to a linear combination of $\chi_{\gamma, \delta}$, the right-hand side is a $\nu$-measurable complex function of $s$.

2. Let $\chi_{\alpha, \beta}$ and $e_{i}$ be as in the proof of 1 . If $\xi$ is an $\mathfrak{h}$-valued $\nu$-measurable map, then $s \rightarrow\langle\psi, \xi(s)\rangle$ is a $\nu$-measurable function for any $\psi \in \mathfrak{h}$. In particular, $s \rightarrow\left\langle\left[\chi_{\alpha, \beta}\right], \xi(s)\right\rangle=\int_{\alpha}^{\beta} \xi(s \mid r) d r$ is a $\nu$-measurable function for any $a<\alpha \leq \beta<b$, i.e., $\xi$ is a $\nu$-measurable family of functions on $(a, b)$. Conversely, if $\xi$ is a $\nu$-measurable family of functions on $(a, b)$, then $s \rightarrow \int_{a}^{b} e_{i}(r) \xi(s \mid r) d r$ is a $\nu$-measurable function for every $i=1,2, \ldots$ If, in addition, $\xi(s) \in \mathfrak{h}$ for $\nu$-a.e. $s$, then $\int_{a}^{b} e_{i}(r) \xi(s \mid r) d r=\left\langle e_{i}, \xi(s)\right\rangle$ and, therefore, $\xi$ is an $\mathfrak{h}$-valued $\nu$-measurable map.

3. Let $\xi$ be a $\nu$-measurable family of functions on $(a, b)$ and $r \in(a, b)$. As $\xi(s)$ is continuous at $r$ for $\nu$-a.e. $s$, we have $\xi(s \mid r)=\lim _{n \rightarrow \infty} n \int_{r-1 / 2 n}^{r+1 / 2 n} \xi(s \mid r) d r$ for $\nu$-a.e. $s$. This implies that $s \rightarrow \xi(s \mid r)$ is a $\nu$-measurable function because $s \rightarrow n \int_{r-1 / 2 n}^{r+1 / 2 n} \xi(s \mid r) d r$ are $\nu$-measurable functions. Conversely, let $s \rightarrow \xi(s \mid r)$ be a $\nu$-measurable function for every $r \in(a, b)$ and let $a<\alpha<\beta<b$. Given $n=1,2, \ldots$ and a function $f$ on $(a, b)$, we denote by $S_{n}(f)$ the Riemann sum $\frac{\beta-\alpha}{n} \sum_{k=1}^{n} f\left(r_{n}^{k}\right)$ for $f$ on $[\alpha, \beta]$, where $r_{n}^{k}=\alpha+(\beta-\alpha) k / n$. As $\xi(s)$ is continuous for $\nu$-a.e. $s, \int_{\alpha}^{\beta} \xi(s \mid r) d r$ is the limit of $S_{n}(\xi(s))$ for $\nu$-a.e. $s$. This means that $s \rightarrow \int_{\alpha}^{\beta} \xi(s \mid r) d r$ is a $\nu$-measurable function because $s \rightarrow S_{n}(\xi(s))$ is a $\nu$-measurable function for every $n$.

4. Clearly, $\eta(s)$ is a continuous function on $(a, b)$ for $\nu$-a.e. $s$ and $s \rightarrow \eta(s \mid r)$ is a $\nu$-measurable function for any $r \in(a, b)$. Hence, the statement follows from 3 .

5. For $\nu$-a.e. $s$, the function $\xi(s)^{\prime}$ is locally integrable and ${ }^{32} \int_{\alpha}^{\beta} \xi^{\prime}(s \mid r) d r=\xi(s \mid \beta)-\xi(s \mid \alpha)$. Hence, the statement follows from 3.

Lemma VII.2. Let $\nu$ be a $\sigma$-finite positive measure and $v$ be a $\nu$-measurable family of real locally squareintegrable functions on $(a, b)$. Then $s \rightarrow L_{v(s)}$ is a $\nu$-measurable family of operators in $\mathfrak{h}$.

Proof. By Lemma VI.2, there is a sequence $\varphi_{1}, \varphi_{2}, \ldots$ of functions in $C_{0}^{\infty}(a, b)$ such that the vectors $\left(\left[\varphi_{j}\right], l_{q} \varphi_{j}\right)$ are dense in $G_{L_{q}}$ for any locally square-integrable real function $q$ on $(a, b)$. For each $j=1,2, \ldots$, let $\xi_{j}$ and $\eta_{j}$ be $\nu$-a.e. defined maps such that $\xi_{j}(s)=\left[\varphi_{j}\right]$ and $\eta_{j}(s)=l_{v(s)} \varphi_{j}$ for $\nu$-a.e. $s$. Since $v(s)$ is locally square integrable for $\nu$-a.e. $s$, the vectors $\left(\xi_{j}(s), \eta_{j}(s)\right)$ are dense in $G_{L_{v(s)}}$ for $\nu$-a.e. $s$. By statements 1 and 5 of Lemma VII.1 $\eta_{j}$ is a $\nu$-measurable family of functions on $(a, b)$, and statement 2 of Lemma VII.1 implies that $\eta_{j}$ is an $\mathfrak{h}$-valued $\nu$-measurable map for any $j=1,2, \ldots$ As $\xi_{j}$ are obviously $\mathfrak{h}$-valued $\nu$-measurable maps for all $j$, we conclude that $s \rightarrow L_{v(s)}$ is a $\nu$-measurable family of operators in $\mathfrak{h}$.

Lemma VII.3. Let $\nu$ and $v$ be as in Lemma VII.2 and $\xi$ be a D-valued $\nu$-a.e. defined map such that

$$
l_{v(s)} \xi(s)=0
$$

for $\nu$-a.e. $s$. Suppose there is $r_{0} \in(a, b)$ such that the functions $s \rightarrow \xi\left(s \mid r_{0}\right)$ and $s \rightarrow \xi^{\prime}\left(s \mid r_{0}\right)$ are $\nu$-measurable. Then $\xi$ is a $\nu$-measurable family of functions on $(a, b)$. 
Proof. Clearly, it suffices to show that $\xi$ is a $\nu$-measurable family of functions on $(\alpha, \beta)$ for any real numbers $\alpha$ and $\beta$ such that $a<\alpha<r_{0}<\beta<b$. We fix such $\alpha$ and $\beta$ and set

$$
A_{N}=\left\{s \in S_{\nu}: \int_{\alpha}^{\beta} v(s \mid r)^{2} d r<N^{2} /(\beta-\alpha)\right\}
$$

for each $N=1,2, \ldots$. By statement 1 of LemmaVII.1, $A_{N}$ is a $\nu$-measurable set. By the Cauchy-Bunyakovsky inequality, we have

$$
\int_{\alpha}^{\beta}|v(s \mid r)| d r<N, \quad s \in A_{N} .
$$

Let $\nu_{N}=\left.\nu\right|_{A_{N}}$. To prove our statement, it suffices to show that the set

$$
Q_{N}=\left\{r \in(\alpha, \beta): s \rightarrow \xi(s \mid r) \text { and } s \rightarrow \xi^{\prime}(s \mid r) \text { are } \nu_{N} \text {-measurable functions }\right\}
$$

coincides with $(\alpha, \beta)$ for all $N=1,2, \ldots$ Indeed, this condition implies that $s \rightarrow \xi(s \mid r)$ is a $\nu_{N}$-measurable function for all $N$ and $r \in(\alpha, \beta)$. Because $S_{\nu} \backslash \bigcup_{N=1}^{\infty} A_{N}$ is a $\nu$-null set, this means that $s \rightarrow \xi(s \mid r)$ is a $\nu$-measurable function for every $r \in(\alpha, \beta)$, and statement 3 of Lemma VII.1 ensures that $\xi$ is a $\nu$-measurable family of functions on $(\alpha, \beta)$. Note that $r_{0} \in Q_{N}$ for all $N$. Hence, to prove the equality $Q_{N}=(\alpha, \beta)$, it suffices to verify that the set $R_{N, r}=\{\rho \in(\alpha, \beta):|\rho-r|<1 / 2 N\}$ is contained in $Q_{N}$ for every $N=1,2, \ldots$ and $r \in Q_{N}$. Fix $N$ and $r \in Q_{N}$ and let $\xi_{0}, \xi_{1}, \ldots$ be $\mathcal{D}$-valued $\nu$-a.e. defined maps such that, for $\nu$-a.e. $s$, the relations

$$
\begin{aligned}
& \xi_{0}(s \mid \rho)=\xi(s \mid r)+\xi^{\prime}(s \mid r)(\rho-r), \\
& \xi_{n}(s \mid \rho)=\xi_{0}(s \mid \rho)+\int_{r}^{\rho} d \rho^{\prime} \int_{r}^{\rho^{\prime}} v(s \mid t) \xi_{n-1}(s \mid t) d t, \quad n=1,2, \ldots,
\end{aligned}
$$

hold for all $\rho \in(a, b)$. As $r \in Q_{N}, \xi_{0}$ is a $\nu_{N}$-measurable family of functions on $(a, b)$, and it follows from statements 1 and 4 of Lemma VII.1 that $\xi_{n}$ is a $\nu_{N}$-measurable family of functions on $(a, b)$ for every $n=0,1, \ldots$. Since $\xi(s)$ satisfies (63) for $\nu$-a.e. $s$, it follows that, for $\nu$-a.e. $s$, the equality

$$
\xi(s \mid \rho)=\xi_{0}(s \mid \rho)+\int_{r}^{\rho} d \rho^{\prime} \int_{r}^{\rho^{\prime}} v(s \mid t) \xi(s \mid t) d t
$$

holds for any $\rho \in(a, b)$. Let $\rho \in R_{N, r}$. It follows from (64), (65) and (66) that

$$
\left|\xi(s \mid \rho)-\xi_{n}(s \mid \rho)\right| \leq\left|\xi(s \mid \rho)-\xi_{n-1}(s \mid \rho)\right| / 2
$$

for $\nu_{N}$-a.e. $s$ and all $n=0,1, \ldots$. We hence have

$$
\left|\xi(s \mid \rho)-\xi_{n}(s \mid \rho)\right| \leq\left|\xi(s \mid \rho)-\xi_{0}(s \mid \rho)\right| / 2^{n}
$$

for $\nu_{N}$-a.e. $s$. This means that, for any $\rho \in R_{N, r}$, the sequence $\xi_{n}(s \mid \rho)$ converges to $\xi(s \mid \rho)$ for $\nu_{N}$-a.e. $s$ and, therefore, $s \rightarrow \xi(s \mid \rho)$ is a $\nu_{N}$-measurable function for every $\rho \in R_{N, r}$. As $\xi^{\prime}(s \mid \rho)$ is the limit of $k(\xi(s \mid \rho+1 / k)-\xi(s \mid \rho))$ as $k \rightarrow \infty$ for $\nu$-a.e. $s$, it also follows that $s \rightarrow \xi^{\prime}(s \mid \rho)$ is a $\nu_{N}$-measurable function for every $\rho \in R_{N, r}$. Hence $R_{N, r} \subset Q_{N}$ and the lemma is proved.

Corollary VII.4. Let $\nu$ and $v$ be as in LemmaVII.2. Then there are $\nu$-measurable families $\xi_{1}$ and $\xi_{2}$ of functions on $(a, b)$ such that $\xi_{1}(s)$ and $\xi_{2}(s)$ are linearly independent real elements of $\mathcal{D}$ satisfying equation (63) for $\nu$-a.e. s.

Proof. Choose $r_{0} \in(a, b)$. Let $\xi_{1}$ and $\xi_{2}$ be $\nu$-a.e. defined $\mathcal{D}$-valued maps such that $\xi_{1}(s)$ and $\xi_{2}(s)$ are solutions of (63) satisfying the conditions

$$
\xi_{1}\left(s \mid r_{0}\right)=\xi_{2}^{\prime}\left(s \mid r_{0}\right)=1, \quad \xi_{1}^{\prime}\left(s \mid r_{0}\right)=\xi_{2}\left(s \mid r_{0}\right)=0
$$

for $\nu$-a.e. $s$. Obviously, $\xi_{1}(s)$ and $\xi_{2}(s)$ are linearly independent for $\nu$-a.e. $s$, and Lemma VII.3 implies that $\xi_{1}$ and $\xi_{2}$ are $\nu$-measurable families of functions on $(a, b)$.

Proposition VII.5. Let $\nu$ and $v$ be as in Lemma VII.2. Suppose v(s) is in l.c.c. at a and in l.p.c. at $b$ for $\nu$-a.e. $s$. If $\xi$ is a $\nu$-measurable family of elements of $\mathcal{D}$ such that $\xi(s)$ is a nontrivial real solution of (63) for $\nu$-a.e. $s$, then $s \rightarrow L_{v(s)}^{\xi(s)}$ is a $\nu$-measurable family of self-adjoint operators in $\mathfrak{h}$. If $\mathcal{R}$ is a $\nu$-measurable family of operators in $\mathfrak{h}$ such that $\mathcal{R}(s)$ is a self-adjoint extension of $L_{v(s)}$ for $\nu$-a.e. $s$, then there exists a $\nu$-measurable family $\xi$ of elements of $\mathcal{D}$ such that $\xi(s)$ is a nontrivial real solution of (63) and $\mathcal{R}(s)=L_{v(s)}^{\xi(s)}$ for $\nu$-a.e. $s$. 
Proof. By Lemma VII.2, $s \rightarrow L_{v(s)}$ is a $\nu$-measurable family of operators in $\mathfrak{h}$. This means that there is a sequence $\zeta_{1}, \zeta_{2}, \ldots$ of $\mathfrak{h} \oplus \mathfrak{h}$-valued $\nu$-measurable maps such that the linear span of $\zeta_{1}(s), \zeta_{2}(s), \ldots$ is dense in the graph $G_{L_{v(s)}}$ of $L_{v(s)}$ for $\nu$-a.e. $s$.

Let $\xi$ be a $\nu$-measurable family of elements of $\mathcal{D}$ such that $\xi(s)$ is a nontrivial real solution of (63) for $\nu$-a.e. $s$ and let $\tau$ be a smooth function on $(a, b)$ that is equal to unity in a neighborhood of $a$ and vanishes in a neighborhood of $b$. Let $\nu$-a.e. defined maps $g$ and $h$ be such that

$$
g(s)=[\tau \xi(s)], \quad h(s)=-\left[\tau^{\prime \prime} \xi(s)+2 \tau^{\prime} \xi^{\prime}(s)\right]
$$

for $\nu$-a.e. $s$. We obviously have $g(s) \in \mathfrak{h}$ and $h(s) \in \mathfrak{h}$ for $\nu$-a.e. $s$ and, therefore, statements 1,2 , and 5 of LemmaVII.1 imply that $g$ and $h$ are $\mathfrak{h}$-valued $\nu$-measurable maps. Let $\zeta$ be an $\mathfrak{h} \oplus \mathfrak{h}$-valued $\nu$-measurable map such that $\zeta(s)=(g(s), h(s))$ for $\nu$-a.e. $s$. Note that

$$
l_{v(s)}(\tau \xi(s))=h(s)
$$

for $\nu$-a.e. $s$ and, therefore, $\tau \xi(s) \in \mathcal{D}_{v(s)}$ for $\nu$-a.e. $s$ (see (41)). Since $\xi(s)$ is a nontrivial real solution of (63) and $\tau \xi(s)$ coincides with $\xi(s)$ in a neighborhood of $a$, it follows from (44) and (50) that $\tau \xi(s) \in$ $Z_{v(s)}^{\xi(s)}$ and $\tau \xi(s) \notin \mathcal{D}_{v(s)}^{0}$ for $\nu$-a.e. $s$. In view of (43), (49), and (67), we conclude that $\zeta(s) \in G_{L_{v(s)}^{\xi(s)}} \backslash G_{L_{v(s)}}$ for $\nu$-a.e. $s$. As $G_{L_{v(s)}^{\xi(s)}} \ominus G_{L_{v(s)}}$ is one-dimensional, this implies that the linear span of the sequence $\zeta(s), \zeta_{1}(s), \zeta_{2}(s), \ldots$ is dense in the graph of $L_{v(s)}^{\xi(s)}$ for $\nu$-a.e. $s$. This means that $s \rightarrow L_{v(s)}^{\xi(s)}$ is a $\nu$ measurable family of operators in $\mathfrak{h}$.

Conversely, let $\mathcal{R}$ be a $\nu$-measurable family of operators in $\mathfrak{h}$ such that $\mathcal{R}(s)$ is a self-adjoint extension of $L_{v(s)}$ for $\nu$-a.e. $s$. Then both $s \rightarrow G_{\mathcal{R}(s)}$ and $s \rightarrow G_{L_{v(s)}}$ are $\nu$-measurable families of subspaces of $\mathfrak{h} \oplus \mathfrak{h}$. By statement 1 of Lemma B.6 $s \rightarrow G_{\mathcal{R}(s)} \ominus G_{L_{v(s)}}$ is also a $\nu$-measurable family of subspaces of $\mathfrak{h} \oplus \mathfrak{h}$. Since $G_{\mathcal{R}(s)} \ominus G_{L_{v(s)}}$ is nontrivial for $\nu$-a.e. $s$, there is an $\mathfrak{h} \oplus \mathfrak{h}$-valued $\nu$-measurable map $\eta$ such that $\eta(s)$ is a nonzero element of $G_{\mathcal{R}(s)} \ominus G_{L_{v(s)}}$ for $\nu$-a.e. $s$. $\operatorname{Let}^{33} Q=\left\{s \in S_{\nu}: \eta(s)=-\overline{\eta(s)}\right\}$ and $\zeta$ be a $\nu$-a.e. defined map such that $\zeta(s)=i \eta(s)$ for $\nu$-a.e. $s \in Q$ and $\zeta(s)=\eta(s)+\overline{\eta(s)}$ for $\nu$-a.e. $s \in S_{\nu} \backslash Q$. Then $\zeta$ is an $\mathfrak{h} \oplus \mathfrak{h}$-valued $\nu$-measurable map such that

$$
\zeta(s)=\overline{\zeta(s)}
$$

and $\zeta(s) \neq 0$ for $\nu$-a.e. $s$. Moreover, since $G_{\mathcal{R}(s)}$ and $G_{L_{v(s)}}$ are both invariant under complex conjugation, we have $\zeta(s) \in G_{\mathcal{R}(s)} \ominus G_{L_{v(s)}}$ for $\nu$-a.e. $s$. Let $g$ and $h$ be $\mathfrak{h}$-valued $\nu$-measurable maps such that $\zeta(s)=(g(s), h(s))$ for $\nu$-a.e. $s$. For $\nu$-a.e. $s$, we have

$$
g(s) \in D_{\mathcal{R}(s)} \backslash D_{L_{v(s)}} .
$$

As $g(s) \in D_{\mathcal{R}(s)}$ for $\nu$-a.e. $s$, there exists a $\nu$-a.e. defined map $\tilde{g}$ such that $\tilde{g}(s) \in \mathcal{D}_{v(s)}$ and $g(s)=[\tilde{g}(s)]$ for $\nu$-a.e. $s$. It follows from (68) that $\tilde{g}(s)$ is real for $\nu$-a.e. $s$. By statement 2 of Lemma VII.1, $\tilde{g}$ and $h$ are $\nu$-measurable families of functions on $(a, b)$. Let $\xi$ be a $\nu$-a.e. defined map such that $\xi(s)=\gamma_{v(s)}^{\tilde{g}(s)}$ for $\nu$-a.e. $s$. In view of (69), it follows from Lemma VI.1 that $\xi(s)$ is a nontrivial real solution of (63) and $\mathcal{R}(s)=L_{v(s)}^{\xi(s)}$ for $\nu$-a.e. $s$. Let $\xi_{1}$ and $\xi_{2}$ be as in Corollary VII.4. Since $h(s)=\mathcal{R}(s) g(s)$, we have $h(s)=l_{v(s)} \tilde{g}(s)$ for $\nu$-a.e. $s$. Equations (46) and (47) imply that, for $\nu$-a.e. $s$, equality

$$
\xi(s \mid r)=\tilde{g}(s \mid r)-\frac{1}{W\left(\xi_{1}(s), \xi_{2}(s)\right)}\left[\xi_{1}(s \mid r) \int_{a}^{r} h(s \mid \rho) \xi_{2}(s \mid \rho) d \rho-\xi_{2}(s \mid r) \int_{a}^{r} h(s \mid \rho) \xi_{1}(s \mid \rho) d \rho\right]
$$

holds for all $r \in(a, b)$. By statements 3 and 5 of Lemma VII.1, $s \rightarrow W\left(\xi_{1}(s), \xi_{2}(s)\right)$ is a $\nu$-measurable function. It therefore follows from statements 1 and 4 of Lemma VII.1 that $\xi$ is a $\nu$-measurable family of functions on $(a, b)$.

\section{SELF-ADJOINT EXTENSIONS OF THE THREE-DIMENSIONAL AHARONOV-BOHM HAMILTONIAN}

In this section, we use the results of Secs. [V] and [VII to represent the self-adjoint extensions of $H^{\phi}$ commuting with all operators $T_{G}, G \in \mathcal{G}$, as direct integrals of one-dimensional Schrödinger operators. Let $\mathfrak{h}=L^{2}\left(\mathbb{R}_{+}\right)$and the positive Borel measure $\nu_{0}$ on $S=\mathbb{Z} \times \mathbb{R}$ be as in Sec. II] We begin by constructing 
a suitable unitary operator $V: L_{2}\left(\mathbb{R}^{3}\right) \rightarrow L_{2}\left(S, \mathfrak{h}, \nu_{0}\right)$ and then prove that $\left(\nu_{0}, \mathcal{I}_{\mathfrak{h}, \nu_{0}}, V\right)$ is actually an exact diagonalization for the set of the operators $T_{G}$. After that, we use Proposition $V .3$ to obtain a representation of form (44) for $H^{\phi}$. Finally, we combine Corollary V.2 and Proposition VII.5 to get an explicit description of the self-adjoint extensions of $H^{\phi}$ commuting with $T_{G}$.

We denote by $\lambda_{+}$the restriction to $\mathbb{R}_{+}$of the Lebesgue measure $\lambda$ on $\mathbb{R}$. For $\Phi \in C_{0}^{\infty}(\mathscr{O})$, let the map $\tilde{\Phi}$ from $S$ to $C_{0}^{\infty}\left(\mathbb{R}_{+}\right)$be defined by the relation

$$
\tilde{\Phi}(s \mid r)=\frac{\sqrt{r}}{2 \pi} \int_{-\infty}^{\infty} d x_{3} \int_{0}^{2 \pi} d \varphi \Phi\left(r \cos \varphi, r \sin \varphi, x_{3}\right) e^{-i p x_{3}-i m \varphi}
$$

for any $s=(m, p) \in S$ and $r>0$.

Lemma VIII.1. There is a unique unitary operator $V: L_{2}\left(\mathbb{R}^{3}\right) \rightarrow L_{2}\left(S, \mathfrak{h}, \nu_{0}\right)$ such that the equality

$$
\left(V[\Phi]_{\Lambda}\right)(s)=[\tilde{\Phi}(s)]_{\lambda_{+}}
$$

holds for $\nu_{0}$-a.e. $s$ for any $\Phi \in C_{0}^{\infty}(\mathscr{O})$.

Proof. Let $\Phi \in C_{0}^{\infty}(\mathscr{O})$. Then $s \rightarrow \int_{c}^{d} \tilde{\Phi}(s \mid r) d r$ is a continuous and, hence, $\nu_{0}$-measurable function for any $c, d \in \mathbb{R}_{+}$. This means that $\tilde{\Phi}$ is a $\nu_{0}$-measurable family of functions on $(0, \infty)$, and statement 2 of Lemma VII.1 implies that $s \rightarrow[\tilde{\Phi}(s)]_{\lambda_{+}}$is an $\mathfrak{h}$-valued $\nu_{0}$-measurable map. Let $f$ be the function on $\mathbb{R}^{3}$ defined by the formula

$$
f\left(x_{1}, x_{2}, p\right)=\int_{-\infty}^{\infty} \Phi\left(x_{1}, x_{2}, x_{3}\right) e^{-i p x_{3}} d x_{3} .
$$

Clearly, $f$ is continuous and, hence, Lebesgue measurable. It follows from the Fubini theorem and the Parseval identity for the one-dimensional Fourier transformation that $f$ is square-integrable and

$$
\int\left|f\left(x_{1}, x_{2}, p\right)\right|^{2} d x_{1} d x_{2} d p=2 \pi \int\left|\Phi\left(x_{1}, x_{2}, x_{3}\right)\right|^{2} d x_{1} d x_{2} d x_{3}
$$

Let $g$ be the continuous function on $\mathbb{R} \times \mathbb{R} \times \mathbb{R}_{+}$defined by the relation $g(\varphi, p, r)=f(r \cos \varphi, r \sin \varphi, p)$. By the Fubini theorem, we have

$$
\tilde{\Phi}(s \mid r)=\frac{\sqrt{r}}{2 \pi} \int_{0}^{2 \pi} g(\varphi, p, r) e^{-i m \varphi} d \varphi
$$

for any $s=(m, p) \in S$ and $r \in \mathbb{R}_{+}$. Further, by the Fubini theorem and Parseval identity for the Fourier series expansion, we have

$$
\begin{aligned}
\int d \nu_{0}(s) \int_{0}^{\infty}|\tilde{\Phi}(s \mid r)|^{2} d r & =\int_{0}^{\infty} d r \int_{-\infty}^{\infty} d p \sum_{m \in \mathbb{Z}}|\tilde{\Phi}(m, p \mid r)|^{2}= \\
& =\frac{1}{2 \pi} \int_{0}^{\infty} r d r \int_{-\infty}^{\infty} d p \int_{0}^{2 \pi}|g(\varphi, p, r)|^{2} d \varphi=\frac{1}{2 \pi} \int\left|f\left(x_{1}, x_{2}, p\right)\right|^{2} d x_{1} d x_{2} d p
\end{aligned}
$$

and in view of (73), we conclude that $s \rightarrow[\tilde{\Phi}(s)]_{\lambda_{+}}$is a square-integrable map and

$$
\int\left\|[\tilde{\Phi}(s)]_{\lambda_{+}}\right\|^{2} d \nu_{0}(s)=\int d \nu_{0}(s) \int_{0}^{\infty}|\tilde{\Phi}(s \mid r)|^{2} d r=\int\left|\Phi\left(x_{1}, x_{2}, x_{3}\right)\right|^{2} d x_{1} d x_{2} d x_{3} .
$$

Since $[\Phi]_{\Lambda}$ with $\Phi \in C_{0}^{\infty}(\mathscr{O})$ are dense in $L_{2}\left(\mathbb{R}^{3}\right)$, it follows that there exists a unique isometric operator $V: L_{2}\left(\mathbb{R}^{3}\right) \rightarrow L_{2}\left(S, \mathfrak{h}, \nu_{0}\right)$ satisfying (72). It remains to check that the image of $V$ is the entire space $L_{2}\left(S, \mathfrak{h}, \nu_{0}\right)$. For any $\xi \in L_{2}\left(S, \mathfrak{h}, \nu_{0}\right)$ and $h \in \mathfrak{h}, s \rightarrow\langle\xi(s), h\rangle$ is a $\nu_{0}$-square integrable function because $|\langle\xi(s), h\rangle| \leq\|\xi(s)\|\|h\|$ for $\nu_{0}$-a.e. $s$. Given $\psi \in C_{0}^{\infty}\left(\mathbb{R}_{+}\right), \xi \in L_{2}\left(S, \mathfrak{h}, \nu_{0}\right)$, and $m \in \mathbb{Z}$, we denote by $F_{\xi, \psi, m}$ the element of $L_{2}(\mathbb{R})$ such that $F_{\xi, \psi, m}(p)=\left\langle\xi(m, p),[\psi]_{\lambda_{+}}\right\rangle$for $\lambda$-a.e. $p$. For $\psi \in C_{0}^{\infty}\left(\mathbb{R}_{+}\right)$, $\chi \in C_{0}^{\infty}(\mathbb{R})$, and $m \in \mathbb{Z}$, let $\Phi_{\psi, \chi, m} \in C_{0}^{\infty}(\mathscr{O})$ be such that

$$
\Phi_{\psi, \chi, m}\left(r \cos \varphi, r \sin \varphi, x_{3}\right)=\frac{1}{\sqrt{r}} \psi(r) \chi\left(x_{3}\right) e^{i m \varphi}
$$

for any $r>0$ and $x_{3}, \varphi \in \mathbb{R}$. Then we have

$$
\tilde{\Phi}_{\psi, \chi, m}(k, p \mid r)=\delta_{k m} \hat{\chi}(p) \psi(r),
$$


where $\delta_{k m}=0$ for $k \neq m$ and $\delta_{k m}=1$ for $k=m$ and $\hat{\chi}(p)=\int \chi\left(x_{3}\right) e^{-i p x_{3}} d x_{3}$ is the Fourier transform of $\chi$. It follows that

$$
\left\langle\xi, V\left[\Phi_{\psi, \chi, m}\right]_{\Lambda}\right\rangle_{L_{2}\left(S, \mathfrak{h}, \nu_{0}\right)}=\int \hat{\chi}(p)\left\langle\xi(m, p),[\psi]_{\lambda_{+}}\right\rangle_{\mathfrak{h}} d p=\left\langle\bar{F}_{\xi, \psi, m},[\hat{\chi}]_{\lambda}\right\rangle_{L_{2}(\mathbb{R})}
$$

for every $\xi \in L_{2}\left(S, \mathfrak{h}, \nu_{0}\right)$. Suppose now that $\xi$ is orthogonal to every element of $\operatorname{Im} V$. Since $[\hat{\chi}]_{\lambda}$ with $\chi \in C_{0}^{\infty}(\mathbb{R})$ are dense in $L_{2}(\mathbb{R})$, equation (76) implies that $F_{\xi, \psi, m}=0$, i.e., $\left\langle\xi(s),[\psi]_{\lambda_{+}}\right\rangle=0$ for $\nu_{0}$-a.e. $s$ for any $\psi \in C_{0}^{\infty}\left(\mathbb{R}_{+}\right)$. As $[\psi]_{\lambda_{+}}$with $\psi \in C_{0}^{\infty}\left(\mathbb{R}_{+}\right)$are dense in $\mathfrak{h}$, it follows that $\xi=0$ and, therefore, $\operatorname{Im} V=L_{2}\left(S, \mathfrak{h}, \nu_{0}\right)$.

Lemma VIII.2. Let $V$ be as in LemmaVIII.1. Then $\left(\nu_{0}, \mathcal{I}_{\mathfrak{h}, \nu_{0}}, V\right)$ is an exact diagonalization for the set of the operators $T_{G}$ with $G \in \mathcal{G}$.

Proof. For $\alpha, \beta \in \mathbb{R}$, let $G_{\alpha \beta} \in \mathcal{G}$ be defined by (35). Clearly, each element of $\mathcal{G}$ is equal to $G_{\alpha \beta}$ for some $\alpha, \beta \in \mathbb{R}$. We have

$$
\widetilde{\Phi \circ G_{\alpha \beta}^{-1}}(m, p)=e^{-i \alpha m-i \beta p} \tilde{\Phi}(m, p), \quad(m, p) \in S,
$$

for any $\Phi \in C_{0}^{\infty}(\mathscr{O})$ and, therefore,

$$
V T_{G_{\alpha \beta}} V^{-1}=\mathcal{T}_{\gamma_{\alpha \beta}, \mathfrak{S}}^{\nu_{\alpha}}
$$

where $\mathfrak{S}=\mathcal{I}_{\mathfrak{h}, \nu_{0}}$ and the function $\gamma_{\alpha \beta}$ on $S$ is given by

$$
\gamma_{\alpha \beta}(m, p)=e^{-i \alpha m-i \beta p}
$$

Suppose $(m, p)$ and $\left(m^{\prime}, p^{\prime}\right)$ are such that $\gamma_{\alpha \beta}(m, p)=\gamma_{\alpha \beta}\left(m^{\prime}, p^{\prime}\right)$ for all $(\alpha, \beta) \in \mathbb{Q}^{2}$, where $\mathbb{Q}$ is the set of rational numbers. Then we have

$$
e^{i \alpha\left(m-m^{\prime}\right)+i \beta\left(p-p^{\prime}\right)}=1
$$

for all $(\alpha, \beta) \in \mathbb{Q}^{2}$. Since $\mathbb{Q}^{2}$ is dense in $\mathbb{R}^{2}$, it follows that $m=m^{\prime}$ and $p=p^{\prime}$. This means that the family $\left\{\gamma_{\alpha, \beta}\right\}_{(\alpha, \beta) \in \mathbb{Q}^{2}}$ separates the points of $S$. The desired statement now follows from Theorem IV.2.

The next lemma gives a representation of form (4) for $H^{\phi}$.

Lemma VIII.3. Let $\phi \in \mathbb{R}, V$ be as in LemmaVIII.1, and $\mathcal{H}^{\phi}$ be the map on $S$ such that

$$
\mathcal{H}^{\phi}(m, p)=h_{m+\phi}+p^{2} 1_{\mathfrak{h}}, \quad(m, p) \in S,
$$

where $1_{\mathfrak{h}}$ is the identity operator in $\mathfrak{h}$ and the operator $h_{\kappa}, \kappa \in \mathbb{R}$, is given by (53). Then $\mathcal{H}^{\phi}$ is a $\nu_{0}$-measurable family of operators in $\mathfrak{h}$ and we have

$$
H^{\phi}=V^{-1} \int^{\oplus} \mathcal{H}^{\phi}(s) d \nu_{0}(s) V .
$$

Proof. It easily follows from (17) that

$$
\left(\mathscr{H}^{\phi} \Phi\right)\left(r \cos \varphi, r \sin \varphi, x_{3}\right)=\left(-\partial_{x_{3}}^{2}-\partial_{r}^{2}-\frac{1}{r} \partial_{r}-\frac{1}{r^{2}}\left(\partial_{\varphi}^{2}+2 i \phi \partial_{\varphi}-\phi^{2}\right)\right) F_{\Phi}\left(r, \varphi, x_{3}\right)
$$

for any $\Phi \in C_{0}^{\infty}(\mathscr{O})$, where the operator $\mathscr{H}^{\phi}$ in $C_{0}^{\infty}(\mathscr{O})$ is given by (7) and $F_{\Phi}$ is the smooth function on $\mathbb{R} \times \mathbb{R} \times \mathbb{R}_{+}$which represents $\Phi$ in the cylindrical coordinates,

$$
F_{\Phi}\left(r, \varphi, x_{3}\right)=\Phi\left(r \cos \varphi, r \sin \varphi, x_{3}\right)
$$

Substituting (79) in (71) and integrating by parts yields

$$
\widetilde{\mathscr{H}^{\phi}} \Phi(s)=-\tilde{\Phi}(s)^{\prime \prime}+q_{m+\phi} \tilde{\Phi}(s)+p^{2} \tilde{\Phi}(s)
$$

for every $s=(m, p) \in S$, where $q_{\kappa}, \kappa \in \mathbb{R}$, is given by (52). Since $\tilde{\Phi}(s) \in C_{0}^{\infty}\left(\mathbb{R}_{+}\right)$for all $s \in S$, it follows from (72) and (53) that $\left(V[\Phi]_{\Lambda}\right)(s) \in D_{\mathcal{H}^{\phi}(s)}$ and

$$
\left.\mathcal{H}^{\phi}(s)\left(V[\Phi]_{\Lambda}\right)(s)=l_{q_{m+\phi}} \tilde{\Phi}(s)+p^{2}[\tilde{\Phi}(s)]_{\lambda_{+}}=\widetilde{\left[\mathscr{H}^{\phi} \Phi\right.}(s)\right]_{\lambda_{+}}=\left(V\left[\mathscr{H}^{\phi} \Phi\right]_{\Lambda}\right)(s)=\left(V \check{H}^{\phi}[\Phi]_{\Lambda}\right)(s)
$$


for $\nu_{0}$-a.e. $s=(m, p)$. Since every element of $D_{\check{H}^{\phi}}$ is equal to $[\Phi]_{\Lambda}$ for some $\Phi \in C_{0}^{\infty}(\mathscr{O})$, this implies that $V^{-1} \int^{\oplus} \mathcal{H}^{\phi}(s) d \nu_{0}(s) V$ is an extension of $\check{H}^{\phi}$. In view of (53), Lemma VI.2 implies the existence of a sequence $f_{1}, f_{2}, \ldots$ of elements of $C_{0}^{\infty}\left(\mathbb{R}_{+}\right)$such that $\left(\left[f_{j}\right]_{\lambda_{+}}, l_{q_{\kappa}} f_{j}\right)$ are dense in the graph of $h_{\kappa}$ for any $\kappa \in \mathbb{R}$. Since $p^{2} 1_{\mathfrak{h}}$ is a bounded everywhere defined operator in $\mathfrak{h}$, it follows that the elements $\left(\left[f_{j}\right]_{\lambda_{+}}, \mathcal{H}^{\phi}(s)\left[f_{j}\right]_{\lambda_{+}}\right)$are dense in the graph of $\mathcal{H}^{\phi}(s)$ for all $s \in S$. Let $\chi$ be a nonzero element of $C_{0}^{\infty}(\mathbb{R})$ and $\hat{\chi}(p)=\int_{-\infty}^{\infty} \chi(x) e^{-i p x} d x$ be its Fourier transform. For every $j=1,2, \ldots$ and $m \in \mathbb{Z}$, we define $\xi_{j m}$ to be the $\nu_{0}$-equivalence class such that

$$
\xi_{j m}(k, p)=\delta_{k m} \hat{\chi}(p)\left[f_{j}\right]_{\lambda_{+}}
$$

for $\nu_{0}$-a.e. $(k, p)$, where $\delta_{k m}=0$ for $k \neq m$ and $\delta_{k m}=1$ for $k=m$. Let $\Phi_{j m} \in C_{0}^{\infty}(\mathscr{O})$ be defined by the relation $\Phi_{j m}=\Phi_{f_{j}, \chi, m}$, where $\Phi_{\psi, \chi, m}$ for $\psi \in C_{0}^{\infty}\left(\mathbb{R}_{+}\right)$is given by (74). It follows from (172) and (75) that $\left(V\left[\Phi_{j m}\right]_{\Lambda}\right)(s)=\xi_{j m}(s)$ for $\nu_{0}$-a.e. $s$ and, hence, $V\left[\Phi_{j m}\right]_{\Lambda}=\xi_{j m}$. Thus, $\xi_{j m} \in V\left(D_{\check{H}^{\phi}}\right)$ for all $j=1,2, \ldots$ and $m \in \mathbb{Z}$. Since $\hat{\chi}$ is the restriction to $\mathbb{R}$ of a nontrivial entire function, the set of its zeros is at most countable. This implies that the elements $\left(\xi_{j m}(s), \mathcal{H}^{\phi}(s) \xi_{j m}(s)\right)$ are dense in the graph of $\mathcal{H}^{\phi}(s)$ for $\nu_{0}$-a.e. $s$. The statement of the lemma therefore follows from (8) and Proposition V.3.

Let $\phi \in \mathbb{R}$ and $\theta$ be a Borel real function on $A^{\phi}$, where $A^{\phi}$ is given by (9). We denote by $\mathcal{H}_{\theta}^{\phi}$ the operator-valued map on $S$ such that

$$
\mathcal{H}_{\theta}^{\phi}(s)=\left\{\begin{array}{cc}
\mathcal{H}^{\phi}(s), & s \in S \backslash A^{\phi}, \\
h_{m+\phi, \theta(s)}+p^{2} 1_{\mathfrak{h}}, & s \in A^{\phi},
\end{array}\right.
$$

for every $s=(m, p) \in S$, where $\mathcal{H}^{\phi}$ is defined by (78) and the operator $h_{\kappa, \vartheta}$ for $-1<\kappa<1$ and $\vartheta \in \mathbb{R}$ is given by (61).

Lemma VIII.4. Let $\phi \in \mathbb{R}$. For any Borel real function $\theta$ on $A^{\phi}, \mathcal{H}_{\theta}^{\phi}$ is a $\nu_{0}$-measurable family of selfadjoint operators in $\mathfrak{h}$ such that $\mathcal{H}_{\theta}^{\phi}(s)$ is an extension of $\mathcal{H}^{\phi}(s)$ for every $s \in S$. If $\tilde{\mathcal{H}}$ is a $\nu_{0}$-measurable family of operators in $\mathfrak{h}$ such that $\tilde{\mathcal{H}}(s)$ is a self-adjoint extension of $\mathcal{H}^{\phi}(s)$ for $\nu_{0}$-a.e. s, then $\tilde{\mathcal{H}}$ is $\nu_{0}$-equivalent to $\mathcal{H}_{\theta}^{\phi}$ for some Borel real function $\theta$ on $A^{\phi}$.

Proof. Let $v$ be the map on $S$ defined by by the relation

$$
v(m, p)=q_{m+\phi}, \quad(m, p) \in S,
$$

where $q_{\kappa}, \kappa \in \mathbb{R}$, is given by (52). Then $v$ is a $\nu_{0}$-measurable family of functions on $\mathbb{R}_{+}$, and (53) implies that

$$
L_{v(s)}=h_{m+\phi}
$$

for any $s=(m, p) \in S$. Clearly, $v(s)$ is in l.c.c. at 0 if and only if $s \in A^{\phi}$. Given a Borel real function $\theta$ on $A^{\phi}$, we define the map $\xi_{\theta}$ on $A^{\phi}$ by setting

$$
\xi_{\theta}(s)=u^{m+\phi}(0) \cos \left(\theta(s)-\vartheta_{m+\phi}\right)+w^{m+\phi}(0) \sin \left(\theta(s)-\vartheta_{m+\phi}\right)
$$

for every $s=(m, p) \in A^{\phi}$, where $u^{\kappa}, w^{\kappa}$, and $\vartheta_{\kappa}$ are given by (13), (58), and (17) respectively. By (54), (59), and (83), we have $l_{v(s)} \xi_{\theta}(s)=0$ for every $s \in A^{\phi}$. It follows from (57), (61), (83), and (85) that

$$
h_{m+\phi, \theta(s)}=L_{v(s)}^{\xi_{\theta}(s)}, \quad s=(m, p) \in A^{\phi} .
$$

Since $\xi_{\theta}$ is obviously a $\left.\nu_{0}\right|_{A^{\phi}}$-measurable family of functions on $\mathbb{R}_{+}$, it follows from Proposition VII.5 that $s \rightarrow h_{m+\phi, \theta(s)}$ is a $\nu_{0}$-measurable family of operators in $\mathfrak{h}$ on $A^{\phi}$. Since $(m, p) \rightarrow p^{2} 1_{\mathfrak{h}}$ is a $\nu_{0^{-}}$ measurable family of operators in $\mathfrak{h}$ by Lemma B.3. the $\nu_{0}$-measurability of $\mathcal{H}_{\theta}^{\phi}$ on $A^{\phi}$ follows from (82) and Lemma B.9. As $\mathcal{H}_{\theta}^{\phi}$ is $\nu_{0}$-measurable on $S \backslash A^{\phi}$ by (82) and Lemma VIII.3, we see that $\mathcal{H}_{\theta}^{\phi}$ is a $\nu_{0}$-measurable family of operators in $\mathfrak{h}$. It follows immediately from (78) and (82) that $\mathcal{H}_{\theta}^{\phi}(s)$ is a self-adjoint extension of $\mathcal{H}^{\phi}(s)$ for every $s \in S$.

Let $\tilde{\mathcal{H}}$ be a $\nu_{0}$-measurable family of operators in $\mathfrak{h}$ such that $\tilde{\mathcal{H}}(s)$ is a self-adjoint extension of $\mathcal{H}^{\phi}(s)$ for $\nu_{0}$-a.e. $s$. Let $\mathcal{R}$ be a $\nu_{0}$-a.e. defined family of operators in $\mathfrak{h}$ such that $\mathcal{R}(s)=\tilde{\mathcal{H}}(s)-p^{2} 1_{\mathfrak{h}}$ for $\nu_{0}$-a.e. $s=(m, p)$. By Lemma B.3. $\mathcal{R}$ is $\nu_{0}$-measurable. In view of (78) and (84), $\mathcal{R}(s)$ is a self-adjoint extension of $L_{v(s)}$ for $\nu_{0}$-a.e. $s$. By Proposition VII.5, there exists a $\nu_{0}$-a.e. defined map $\xi$ on $A^{\phi}$ such that $\xi$ is a

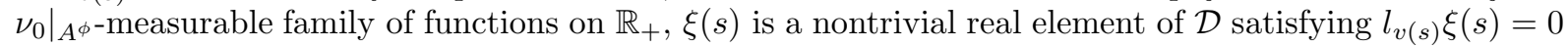


for $\nu_{0}$-a.e. $s \in A^{\phi}$, and $\mathcal{R}(s)=L_{v(s)}^{\xi(s)}$ for $\nu_{0}$-a.e. $s \in A^{\phi}$. By (154), (159), and (60), $u^{m+\phi}(0)$ and $w^{m+\phi}(0)$ are real linearly independent solutions of $l_{v(s)} u^{m+\phi}(0)=l_{v(s)} w^{m+\phi}(0)=0$ for every $s=(m, p) \in A^{\phi}$. Hence, there exist $\nu_{0}$-a.e. defined real functions $C_{1}$ and $C_{2}$ on $A^{\phi}$ such that

$$
\xi(s)=C_{1}(s) u^{m+\phi}(0)+C_{2}(s) w^{m+\phi}(0)
$$

for $\nu_{0}$-a.e. $s=(m, p) \in A^{\phi}$. In view of (60), we have

$$
C_{1}(s)=\frac{\pi}{2} W\left(\xi(s), w^{m+\phi}(0)\right), \quad C_{2}(s)=-\frac{\pi}{2} W\left(\xi(s), u^{m+\phi}(0)\right)
$$

for $\nu_{0}$-a.e. $s=(m, p) \in A^{\phi}$. It follows from statements 3 and 5 of Lemma VII.1 that $C_{1}$ and $C_{2}$ are $\nu_{0}$-measurable functions on $A^{\phi}$. Since $\xi(s) \neq 0$, we have $C_{1}(s)^{2}+C_{2}(s)^{2} \neq 0$ for $\nu_{0}$-a.e. $s \in A^{\phi}$. Let $\theta$ be a Borel function on $A^{\phi}$ such that

$$
\theta(s)=\vartheta_{m+\phi}+\tau\left(C_{2}(s)\right) \arccos \frac{C_{1}(s)}{C_{1}(s)^{2}+C_{2}(s)^{2}}
$$

for $\nu_{0}$-a.e. $s=(m, p) \in A^{\phi}$, where $\tau(y)=1$ for $y \geq 0$ and $\tau(y)=-1$ for $y<0$. We then have

$$
\cos \left(\theta(s)-\vartheta_{m+\phi}\right)=\frac{C_{1}(s)}{C_{1}(s)^{2}+C_{2}(s)^{2}}, \quad \sin \left(\theta(s)-\vartheta_{m+\phi}\right)=\frac{C_{2}(s)}{C_{1}(s)^{2}+C_{2}(s)^{2}}
$$

for $\nu_{0}$-a.e. $s=(m, p) \in A^{\phi}$. This means that $\xi(s)$ is proportional to the function $\xi_{\theta}(s)$ given by (85) and it follows from (86) that $\mathcal{R}(s)=h_{m+\phi, \theta(s)}$ for $\nu_{0}$-a.e. $s=(m, p) \in A^{\phi}$. Hence, $\tilde{\mathcal{H}}(s)=\mathcal{H}_{\theta}^{\phi}(s)$ for $\nu_{0}$-a.e. $s \in A^{\phi}$. As $\mathcal{H}^{\phi}(s)$ is self-adjoint for all $s \in S \backslash A^{\phi}$, we have $\tilde{\mathcal{H}}(s)=\mathcal{H}^{\phi}(s)=\mathcal{H}_{\theta}^{\phi}(s)$ for $\nu_{0}$-a.e. $s \in S \backslash A^{\phi}$. We therefore have $\tilde{\mathcal{H}}(s)=\mathcal{H}_{\theta}^{\phi}(s)$ for $\nu_{0}$-a.e. $s$.

Given $\phi \in \mathbb{R}$ and a Borel real function $\theta$ on $A^{\phi}$, we define the operator $R_{\theta}^{\phi}$ in $L_{2}\left(\mathbb{R}^{3}\right)$ by the relation

$$
R_{\theta}^{\phi}=V^{-1} \int^{\oplus} \mathcal{H}_{\theta}^{\phi}(s) d \nu_{0}(s) V
$$

where the unitary operator $V: L_{2}\left(\mathbb{R}^{3}\right) \rightarrow L_{2}\left(S, \mathfrak{h}, \nu_{0}\right)$ is as in Lemma VIII.1.

Proposition VIII.5. Let $\phi \in \mathbb{R}$. For any Borel real function $\theta$ on $A^{\phi}, R_{\theta}^{\phi}$ is a self-adjoint extension of $H^{\phi}$ commuting with $T_{G}$ for all $G \in \mathcal{G}$. Every self-adjoint extension of $H^{\phi}$ commuting with $T_{G}$ for all $G \in \mathcal{G}$ is equal to $R_{\theta}^{\phi}$ for some Borel real function $\theta$ on $A^{\phi}$. Given Borel real functions $\theta$ and $\tilde{\theta}$ on $A^{\phi}$, we have $R_{\theta}^{\phi}=R_{\tilde{\theta}}^{\phi}$ if and only if $\theta(s)-\tilde{\theta}(s) \in \pi \mathbb{Z}$ for $\nu_{0}$-a.e. $s \in A^{\phi}$.

Proof. If $\theta$ is a Borel real function on $A^{\phi}$, then it follows immediately from Corollary V.2 and Lemmas VIII.2. VIII.3, and VIII.4 that $R_{\theta}^{\phi}$ is a self-adjoint extension of $H^{\phi}$ commuting with $T_{G}$ for all $G \in \mathcal{G}$.

Conversely, let $\tilde{H}$ be a self-adjoint extension of $H^{\phi}$ commuting with $T_{G}$ for all $G \in \mathcal{G}$. By Corollary V.2 and Lemmas VIII.2 and VIII.3, there is a $\nu_{0}$-measurable family $\tilde{\mathcal{H}}$ of operators in $\mathfrak{h}$ such that $\tilde{\mathcal{H}}(s)$ is a self-adjoint extension of $\mathcal{H}^{\phi}(s)$ for $\nu_{0}$-a.e. $s$ and

$$
\tilde{H}=V^{-1} \int^{\oplus} \tilde{\mathcal{H}}(s) d \nu_{0}(s) V
$$

By Lemma VIII.4, there is a Borel real function $\theta$ on $A^{\phi}$ such that $\tilde{\mathcal{H}}(s)=\mathcal{H}_{\theta}^{\phi}(s)$ for $\nu_{0}$-a.e. $s$. In view of (87) and (88), it follows that $\tilde{H}=R_{\theta}^{\phi}$.

Let $\theta$ and $\tilde{\theta}$ be Borel real functions on $A^{\phi}$. By Proposition B.21 the equality $R_{\theta}^{\phi}=R_{\tilde{\theta}}^{\phi}$ holds if and only if $\mathcal{H}_{\theta}^{\phi}(s)=\mathcal{H}_{\tilde{\theta}}^{\phi}(s)$ for $\nu_{0}$-a.e. $s$. By (61) and (82), the latter condition is fulfilled if and only if $u_{\theta(s)}^{m+\phi}(0)$ is proportional to $u_{\tilde{\theta}(s)}^{m+\phi}(0)$ for $\nu_{0}$-a.e. $s=(m, p) \in A^{\phi}$. In view of (57), this is true if and only if $\theta(s)-\tilde{\theta}(s) \in \pi \mathbb{Z}$ for $\nu_{0}$-a.e. $s \in A^{\phi}$. 


\section{DIRECT INTEGRALS OF MEASURES}

Let $\nu$ be a $\sigma$-finite positive measure and $\mu$ be a map such that $\mu(s)$ is a $\sigma$-finite positive measure for $\nu$ a.e. $s$. Suppose $\Sigma$ is a $\sigma$-algebra whose every element is a $\mu(s)$-measurable set for $\nu$-a.e. $s$. Under suitable measurability conditions on $\mu$, we shall define a new measure $M$, called the direct integral of $\mu$ and denoted by $(\Sigma)-\int^{\oplus} \mu(s) d \nu(s)$, satisfying $S_{M}=S_{\nu} \times G$, where $G$ is the largest element of $\Sigma$. This measure has a Fubini-type property (Proposition IX.10) and can be viewed as a generalization of the direct product of measures. The main result of this section (Proposition IX.14) states that the space $L_{2}\left(S_{M}, M\right)$ can be identified with $\int^{\oplus} L_{2}(G, \mu(s)) d \nu(s)$. We shall see in Sec. $\mathbb{X}$ that the measure $\left(\mathcal{B}_{\mathbb{R}}\right)-\int^{\oplus} \mu_{\theta}^{\phi}(s) d \nu_{0}(s)$, where $\mu_{\theta}^{\phi}$ is given by (30) and $\mathcal{B}_{\mathbb{R}}$ is the Borel $\sigma$-algebra on $\mathbb{R}$, satisfies the conditions of Proposition $\amalg .4$ and, therefore, coincides with $M_{\theta}^{\phi}$. This will allow us to obtain a direct integral representation of the space $L_{2}\left(S \times \mathbb{R}, M_{\theta}^{\phi}\right)$ entering Proposition II.1 and relate it to the results of Sec. VIII.

Definition IX.1. Let $\nu$ be a $\sigma$-finite positive measure, $\Sigma$ be a $\sigma$-algebra, and a map $\mu$ be such that $\mu(s)$ is a $\sigma$-finite positive measure for $\nu$-a.e. s. Let the $\delta$-ring $\mathcal{Q}_{\mu, \nu}^{\Sigma}$ be defined by the relation

$$
\mathcal{Q}_{\mu, \nu}^{\Sigma}=\left\{A \in \Sigma: A \in D_{\mu(s)} \text { for } \nu \text {-a.e. } s\right\}
$$

We say that $\mu$ is a $(\nu, \Sigma)$-measurable family of measures if $\Sigma=\sigma\left(\mathcal{Q}_{\mu, \nu}^{\Sigma}\right)$ and $s \rightarrow \mu(s \mid A)$ is a $\nu$-measurable function for any $A \in \mathcal{Q}_{\mu, \nu}^{\Sigma}$.

Lemma IX.2. Let $\nu, \Sigma, \mu$, and $\mathcal{Q}_{\mu, \nu}^{\Sigma}$ be as in Definition IX.1. Let $\mathcal{K} \subset \mathcal{Q}_{\mu, \nu}^{\Sigma}$ be closed under finite intersections and satisfy $\sigma(\mathcal{K})=\Sigma$. Let a map $\xi$ be such that, for every $A \in \mathcal{Q}_{\mu, \nu}^{\Sigma}, \xi(s)$ is a $\mu(s)$-integrable complex function on $A$ for $\nu$-a.e. s. If $s \rightarrow \int_{A} \xi(s \mid E) d \mu(s \mid E)$ is a $\nu$-measurable function for any $A \in \mathcal{K}$, then the same is true for every $A \in \mathcal{Q}_{\mu, \nu}^{\Sigma}$.

If $\xi(s)$ is $\mu(s)$-equivalent to unity for $\nu$-a.e. $s$, this lemma reduces to the next result.

Corollary IX.3. Let $\nu, \Sigma, \mu$, and $\mathcal{K}$ be as in Lemma IX.2. If $s \rightarrow \mu(s \mid A)$ is a $\nu$-measurable function for any $A \in \mathcal{K}$, then $\mu$ is a $(\nu, \Sigma)$-measurable family of measures.

For the proof of Lemma IX.2, we need the next definition.

Definition IX.4. We say that a nonempty set of sets $\mathcal{Q}$ is an $\alpha$-class if it satisfies the following conditions

(1) If $A, B \in \mathcal{Q}$ and $A \cap B=\varnothing$, then $A \cup B \in \mathcal{Q}$.

(2) If $A, B \in \mathcal{Q}$ and $B \subset A$, then $A \backslash B \in \mathcal{Q}$.

(3) If $A_{1} \supset A_{2} \supset \ldots$ is a nonincreasing sequence of elements of $\mathcal{Q}$, then $\bigcap_{i=1}^{\infty} A_{i} \in \mathcal{Q}$.

Remark IX.5. In the context of $\delta$-rings, the role of $\alpha$-classes is essentially the same as that of Dynkin systems (see, e.g., Sec. 2 in Ref. 34) with respect to $\sigma$-algebras. In particular, Lemma IX.6 below is an analogue of Theorem 2.4 in Ref. 34 .

Lemma IX.6. Let $\mathcal{Q}$ be an $\alpha$-class. If there exists a set $\mathcal{K} \subset \mathcal{Q}$ that is closed under finite intersections and satisfies $\mathcal{Q} \subset \sigma(\mathcal{K})$, then $\mathcal{Q}$ is a $\delta$-ring.

Proof of Lemma IX.2. It is straightforward to check that the set

$$
\mathcal{L}=\left\{A \in \mathcal{Q}_{\mu, \nu}^{\Sigma}: s \rightarrow \int_{A} \xi(s \mid E) d \mu(s \mid E) \text { is a } \nu \text {-measurable function }\right\} .
$$

is an $\alpha$-class containing $\mathcal{K}$. As $\sigma(\mathcal{K})=\Sigma$, we have $\mathcal{L} \subset \sigma(\mathcal{K})$, and Lemma IX.6 implies that $\mathcal{L}$ is a $\delta$-ring. As $\sigma(\mathcal{L})=\Sigma$, every $A \in \mathcal{Q}_{\mu, \nu}^{\Sigma}$ is representable as the union of a sequence $A_{1} \subset A_{2} \subset \ldots$ of elements of $\mathcal{L}$. Hence,

$$
\int_{A} \xi(s \mid E) d \mu(s \mid E)=\lim _{k \rightarrow \infty} \int_{A_{k}} \xi(s \mid E) d \mu(s \mid E)
$$

for $\nu$-a.e. $s$ and, therefore, $s \rightarrow \int_{A} \xi(s \mid E) d \mu(s \mid E)$ is $\nu$-measurable. Thus, $\mathcal{Q}_{\mu, \nu}^{\Sigma}=\mathcal{L}$ and the lemma is proved. 
Proof of Lemma IX.6. Let $\mathcal{L}$ be the smallest $\alpha$-class containing $\mathcal{K}{ }^{35}$ We first prove that $\mathcal{L}$ is a $\delta$-ring. To this end, it suffices to show that $\mathcal{L}$ is closed under finite intersections because every $\alpha$-class with this property is a $\delta$-ring. For $A \in \mathcal{L}$, we set

$$
\mathcal{L}^{A}=\{B \in \mathcal{L}: A \cap B \in \mathcal{L}\}
$$

Let $A \in \mathcal{L}$ and $B, C \in \mathcal{L}^{A}$ be such that $B \cap C=\varnothing$. As $(A \cap B) \cap(A \cap C)=\varnothing$ and both sets $A \cap B$ and $A \cap C$ belong to $\mathcal{L}$, the set $A \cap(B \cup C)=(A \cap B) \cup(A \cap C)$ also belongs to $\mathcal{L}$ by condition 1 of Definition IX.4 This means that $B \cup C \in \mathcal{L}^{A}$.

If $B, C \in \mathcal{L}^{A}$ are such that $C \subset B$, then $A \cap(B \backslash C)=(A \cap B) \backslash(A \cap C)$. Since both $A \cap B$ and $A \cap C$ belong to $\mathcal{L}$ and $A \cap C \subset A \cap B$, we have $A \cap(B \backslash C) \in \mathcal{L}$ by condition 2 of Definition IX.4 and, hence, $B \backslash C \in \mathcal{L}^{A}$.

Now let $B_{1} \supset B_{2} \supset \ldots$ be a nonincreasing sequence of elements of $\mathcal{L}^{A}$ and let $B=\bigcap_{i=1}^{\infty} B_{i}$. Since $A \cap B=\bigcap_{i=1}^{\infty}\left(A \cap B_{i}\right)$ and the sets $A \cap B_{1}, A \cap B_{2}, \ldots$ constitute a nonincreasing sequence of elements of $\mathcal{L}$, we have $A \cap B \in \mathcal{L}$ by condition 3 of Definition IX.4 and, therefore, $B \in \mathcal{L}^{A}$.

It follows from the above that $\mathcal{L}^{A}$ is an $\alpha$-class for any $A \in \mathcal{L}$. If $B \in \mathcal{K}$, then we obviously have $\mathcal{K} \subset \mathcal{L}^{B}$ and, hence, $\mathcal{L}^{B}=\mathcal{L}$. It follows that $A \cap B \in \mathcal{L}$ for arbitrary $A \in \mathcal{L}$ and $B \in \mathcal{K}$ and, therefore, $B \in \mathcal{L}^{A}$. This means that $\mathcal{K} \subset \mathcal{L}^{A}$ for every $A \in \mathcal{L}$. Hence, $\mathcal{L}^{A}=\mathcal{L}$ for every $A \in \mathcal{L}$ and, consequently, $A \cap B \in \mathcal{L}$ for every $A, B \in \mathcal{L}$. Thus, $\mathcal{L}$ is a $\delta$-ring.

We now show that $\mathcal{Q}$ is a $\delta$-ring. As for $\mathcal{L}$, it suffices to show that $A \cap B \in \mathcal{Q}$ for any $A, B \in \mathcal{Q}$. As $\mathcal{L}$ is a $\delta$-ring, every element of $\sigma(\mathcal{K})=\sigma(\mathcal{L})$ is a countable union of elements of $\mathcal{L}$. Let $A_{1} \subset A_{2} \subset \ldots$ and $B_{1} \subset B_{2} \subset \ldots$ be nondecreasing sequences of elements of $\mathcal{L}$ such that $A=\bigcup_{i=1}^{\infty} A_{i}$ and $B=\bigcup_{i=1}^{\infty} B_{i}$. As $\mathcal{L}$ is a ring, $C_{i}=A_{i} \cap B_{i}$ belongs to $\mathcal{L}$ (and, hence, to $\mathcal{Q}$ ) for every $i=1,2, \ldots$ It follows from condition 2 of Definition IX.4 that $C_{i}^{\prime}=A \backslash C_{i}$ belongs to $\mathcal{Q}$ for every $i=1,2, \ldots$ As $C_{1}^{\prime} \supset C_{2}^{\prime} \supset \ldots$, condition 3 of Definition IX.4 implies that $A \backslash B=\bigcap_{i=1}^{\infty} C_{i}^{\prime}$ belongs to $\mathcal{Q}$. Applying condition 2 of Definition IX.4 again, we conclude that $A \cap B=A \backslash(A \backslash B)$ belongs to $\mathcal{Q}$.

Given sets of sets $\mathcal{Q}_{1}$ and $\mathcal{Q}_{2}$, we denote by $\mathcal{Q}_{1} \otimes \mathcal{Q}_{2}$ the set of all sets $A_{1} \times A_{2}$, where $A_{1} \in \mathcal{Q}_{1}$ and $A_{2} \in \mathcal{Q}_{2}$. Let $A$ be a set. For any $s$, we define its section $A_{s}$ by the relation $A_{s}=\{E:(s, E) \in A\}$.

Let $\nu$ be a $\sigma$-finite positive measure, $\Sigma$ be a $\sigma$-algebra, and $\mu$ be a $(\nu, \Sigma)$-measurable family of measures. We set $\Xi_{\mu, \nu}^{\Sigma}=\sigma\left(D_{\nu} \otimes \Sigma\right)$ and denote by $\Delta_{\mu, \nu}^{\Sigma}$ the set of all $A \in \Xi_{\mu, \nu}^{\Sigma}$ such that $A_{s} \in D_{\mu(s)}$ for $\nu$-a.e. $s$ and $s \rightarrow \mu\left(s \mid A_{s}\right)$ is a $\nu$-integrable function.

Proposition IX.7. Let $\nu$ be a $\sigma$-finite positive measure, $\Sigma$ be a $\sigma$-algebra, and $\mu$ be a $(\nu, \Sigma)$-measurable family of measures. Then there is a unique $\Xi_{\mu, \nu}^{\Sigma}$-compatible measure $M$ such that $\Delta_{\mu, \nu}^{\Sigma} \subset D_{M}$ and

$$
M(A)=\int \mu\left(s \mid A_{s}\right) d \nu(s)
$$

for any $A \in \Delta_{\mu, \nu}^{\Sigma}$. Moreover, the equality $\Xi_{\mu, \nu}^{\Sigma} \cap D_{M}=\Delta_{\mu, \nu}^{\Sigma}$ is fulfilled. If $\nu$ is $\tilde{\Sigma}$-compatible for some $\sigma$-algebra $\tilde{\Sigma}$, then $M$ is $\sigma(\tilde{\Sigma} \otimes \Sigma)$-compatible.

Proof. Let $\mathcal{Q}=\mathcal{Q}_{\mu, \nu}^{\Sigma}, \Xi=\Xi_{\mu, \nu}^{\Sigma}, \Delta=\Delta_{\mu, \nu}^{\Sigma}$, and $\mathcal{K}=\Delta \cap\left(D_{\nu} \otimes \mathcal{Q}\right)$. Since $\mathcal{Q}$ is a $\delta$-ring, $\mathcal{K}$ is closed under finite intersections. Given $B \in D_{\nu}, C \in \mathcal{Q}$, and $n=1,2, \ldots$, we set $B_{n}=\{s \in B: \mu(s \mid C) \leq n\}$. Clearly, $B=N \cup \bigcup_{n} B_{n}$, where $N$ is a $\nu$-null set. Since $B_{n} \times C \in \mathcal{K}$ for all $n$ and $N \times C \in \mathcal{K}$, we conclude that $B \times C \in \sigma(\mathcal{K})$. Thus, $D_{\nu} \otimes \mathcal{Q} \subset \sigma(\mathcal{K})$. As $\sigma(\mathcal{Q})=\Sigma$, it follows that $\sigma(\mathcal{K})=\Xi$. As $\Delta$ is obviously an $\alpha$-class, Lemma IX.6 implies that $\Delta$ is a $\delta$-ring. Let the function $m: \Delta \rightarrow \mathbb{R}$ be such that $m(A)$ is equal to the right-hand side of (89) for any $A \in \Delta$. It follows from the monotone convergence theorem that $m$ is a $\sigma$-additive function satisfying condition (a) of Sec. A2. Hence, the existence and uniqueness of the measure $M$, as well as the equality $\Xi \cap D_{M}=\Delta$, are ensured by Lemma A.3. Now suppose $\nu$ is $\tilde{\Sigma}$-compatible and let $\mathcal{L}$ denote the set of all $A \in \Xi$ such that there exist $B_{1}, B_{2} \in \sigma(\tilde{\Sigma} \otimes \Sigma)$ satisfying the conditions $B_{1} \subset A \subset B_{2}$ and $M\left(B_{2} \backslash B_{1}\right)=0$. Then $\mathcal{L}$ is a $\sigma$-algebra that contains $D_{\nu} \otimes \Sigma$ and, hence, coincides with $\Xi$. In view of the $\Xi$-compatibility of $M$, this implies that $M$ is $\sigma(\tilde{\Sigma} \otimes \Sigma)$-compatible.

Definition IX.8. Under the conditions of Proposition IX.7 we call the measure $M$ the direct integral of $\mu$ with respect to $(\nu, \Sigma)$ and denote it by $(\Sigma)-\int^{\oplus} \mu(s) d \nu(s)$.

Lemma IX.9. Let $\nu$ be a $\sigma$-finite positive measure, $\Sigma$ be a $\sigma$-algebra, $\mu$ be a $(\nu, \Sigma)$-measurable family of measures, and $M=(\Sigma)-\int^{\oplus} \mu(s) d \nu(s)$. An $M$-measurable set $N$ is an $M$-null set if and only if $N_{s}$ is a $\mu(s)$-null set for $\nu$-a.e. $s$. 
Proof. Let $\Xi=\Xi_{\mu, \nu}^{\Sigma}$ and $N$ be an $M$-measurable set. By the $\Xi$-compatibility of $M$, there is a set $N^{\prime} \in \Xi$ such that $N^{\prime} \supset N$ and $N^{\prime} \backslash N$ is an $M$-null set. If $N$ is an $M$-null set, then $N^{\prime}$ is also an $M$-null set, and it follows from Proposition IX.7 that $N_{s}^{\prime} \in D_{\mu(s)}$ for $\nu$-a.e. $s$ and $\int \mu\left(s \mid N_{s}\right) d \nu(s)=0$, i.e., $N_{s}^{\prime}$ is a $\mu(s)$-null set for $\nu$-a.e. $s$. Since $N_{s} \subset N_{s}^{\prime}$, this implies that $N_{s}$ is a $\mu(s)$-null set for $\nu$-a.e. $s$. Conversely, suppose $N_{s}$ is a $\mu(s)$-null set for $\nu$-a.e. $s$. By the above $\left(N^{\prime} \backslash N\right)_{s}$ is a $\mu(s)$-null set and, hence, $N_{s}^{\prime}$ is a $\mu(s)$-null set for $\nu$-a.e. $s$. Proposition IX.7 now implies that $M\left(N^{\prime}\right)=0$ and, therefore, $N$ is an $M$-null set.

Proposition IX.10. Let $\nu, \Sigma, \mu$, and $M$ be as in LemmaIX.9. For any $M$-integrable complex function $f$, the function $E \rightarrow f(s, E)$ is $\mu(s)$-integrable for $\nu$-a.e. $s$ and we have

$$
\int f(s, E) d M(s, E)=\int d \nu(s) \int f(s, E) d \mu(s \mid E) .
$$

Proof. Let $\Xi=\Xi_{\mu, \nu}^{\Sigma}$. As $M$ is $\Xi$-compatible, every $A \in D_{M}$ is representable in the form $A=B \cup N$, where $B \in D_{M} \cap \Xi$ and $N$ is an $M$-null set. By Proposition IX.7 we have $B \in \Delta_{\mu, \nu}^{\Sigma}$. Hence, $B_{s} \in D_{\mu(s)}$ for $\nu$-a.e. $s$, the function $s \rightarrow \mu\left(s \mid B_{s}\right)$ is $\nu$-integrable, and $M(B)=\int \mu\left(s \mid B_{s}\right) d \nu(s)$. Since $A_{s}=B_{s} \cup N_{s}$, it follows from Lemma IX.9 that $A_{s} \in D_{\mu(s)}$ and $\mu\left(s \mid A_{s}\right)=\mu\left(s \mid B_{s}\right)$ for $\nu$-a.e. $s$. Hence, the function $s \rightarrow \mu\left(s \mid A_{s}\right)$ is $\nu$-integrable, and $M(A)=\int \mu\left(s \mid A_{s}\right) d \nu(s)$. This proves the proposition for $f=X_{A}$, where $X_{A}$ is the function on $S_{M}$ that is equal to unity on $A$ and vanishes on $S_{M} \backslash A$. We say that a function $f$ on $S_{M}$ is simple if it can be represented in the form $f=\sum_{i=1}^{n} c_{i} X_{A_{i}}$ with $c_{i} \in \mathbb{C}$ and $A_{i} \in D_{M}$. Clearly, the proposition is true if $f$ is a simple function. If $f$ is a nonnegative $M$-integrable function, then there is a nondecreasing sequence $f_{k}$ of nonnegative simple functions such that $f_{k}(s, E) \leq f(s, E)$ and $f_{k}(s, E) \rightarrow f(s, E)$ as $k \rightarrow \infty$ for $M$-a.e. $(s, E)$. Let $g_{k}$ be a $\nu$-integrable function such that $g_{k}(s)=\int f_{k}(s, E) d \mu(s \mid E)$ for $\nu$-a.e. $s$. The dominated convergence theorem implies that

$$
\int f(s, E) d M(s, E)=\lim _{k \rightarrow \infty} \int f_{k}(s, E) d M(s, E)=\lim _{k \rightarrow \infty} \int g_{k}(s) d \nu(s) .
$$

By the monotone convergence theorem, there exists a $\nu$-integrable function $g$ such that $g(s)=$ $\lim _{k \rightarrow \infty} g_{k}(s)$ for $\nu$-a.e. $s$, and we have

$$
\int f(s, E) d M(s, E)=\int g(s) d \nu(s) .
$$

By Lemma IX.9, for $\nu$-a.e. $s$, the relations $f_{k}(s, E) \leq f(s, E)$ and $f_{k}(s, E) \rightarrow f(s, E)$ hold for $\mu(s)$-a.e. $E$. In view of the existence of $\lim _{k \rightarrow \infty} g_{k}(s)$, the monotone convergence theorem implies that the function $E \rightarrow f(s, E)$ is $\mu(s)$-integrable and $\int f(s, E) d \mu(s \mid E)=g(s)$ for $\nu$-a.e. $s$. Substituting this equality in (91) yields (90). To complete the proof, it remains to note that every $M$-integrable function is a linear combination of nonnegative $M$-integrable functions.

Corollary IX.11. Let $\nu, \Sigma, \mu$, and $M$ be as in LemmaIX.9 and $f$ be an $M$-measurable complex function. Then $E \rightarrow f(s, E)$ is a $\mu(s)$-measurable complex function for $\nu$-a.e. s. If $E \rightarrow f(s, E)$ is $\mu(s)$-integrable for $\nu$-a.e. $s$, then $s \rightarrow \int f(s, E) d \mu(s \mid E)$ is a $\nu$-measurable function. If, in addition, $f$ is nonnegative and $s \rightarrow \int f(s, E) d \mu(s \mid E)$ is a $\nu$-integrable function, then $f$ is $M$-integrable.

Proof. Let $A_{1} \subset A_{2} \subset \ldots$ be a sequence of elements of $D_{M}$ such that $S_{M}=\bigcup_{k=1}^{\infty} A_{k}$. Let $B_{k}=$ $A_{k} \cap\left\{(s, E) \in S_{M}:|f(s, E)| \leq k\right\}$ and $f_{k}=X_{B_{k}} f$, where $X_{B_{k}}$ is as in the proof of Proposition IX.10. Then $S_{M}$ coincides with $\bigcup_{k=1}^{\infty} B_{k}$ up to an $M$-null set and, therefore, $f_{k}(s, E) \rightarrow f(s, E)$ as $k \rightarrow \infty$ for $M$-a.e. $(s, E)$. Since $f_{k}$ are $M$-integrable for all $k$, Lemma IX.9 and Proposition IX.10 imply that, for $\nu$ a.e. $s$, the functions $E \rightarrow f_{k}(s, E)$ are $\mu(s)$-integrable and converge $\mu(s)$-a.e. to the function $E \rightarrow f(s, E)$. Hence, the latter is $\mu(s)$-measurable for $\nu$-a.e. $s$. If it is also $\mu(s)$-integrable for $\nu$-a.e. $s$, then it follows from the dominated convergence theorem that $\int f(s, E) d \mu(s \mid E)=\lim _{k \rightarrow \infty} \int f_{k}(s, E) d \mu(s \mid E)$ for $\nu$-a.e. $s$. Since $s \rightarrow \int f_{k}(s, E) d \mu(s \mid E)$ are $\nu$-measurable functions by Proposition IX.10 we conclude that $s \rightarrow \int f(s, E) d \mu(s \mid E)$ is $\nu$-measurable. If $f$ is nonnegative and the function $s \rightarrow \int f(s, E) d \mu(s \mid E)$ is $\nu$-integrable, then Proposition IX.10 implies that

$$
\int f_{k}(s, E) d M(s, E) \leq \int d \nu(s) \int f(s, E) d \mu(s \mid E)
$$

for all $k$. The $M$-integrability of $f$ therefore follows from the monotone convergence theorem.

Lemma IX.12. Let $\mathcal{K}$ be a set of sets closed under finite intersections and $\nu$ be a positive $\sigma$-finite $\sigma(\mathcal{K})$ compatible measure. Suppose $f$ is a $\nu$-measurable complex function that is $\nu$-integrable on every set in $\mathcal{K}$. If $\int_{A} f(s) d \nu(s)=0$ for every $A \in \mathcal{K}$, then $f(s)=0$ for $\nu$-a.e. $s$. 
Proof. If $f$ satisfies the conditions of the lemma, then the same is true for its real and imaginary parts. So we can assume that $f$ is real. Let

$$
\mathcal{Q}=\left\{A \in \sigma(\mathcal{K}): f \text { is } \nu \text {-integrable on } A \text { and } \int_{A} f(s) d \nu(s)=0\right\} .
$$

Clearly, $\mathcal{Q}$ is an $\alpha$-class satisfying $\mathcal{K} \subset \mathcal{Q} \subset \sigma(\mathcal{K})$ and, therefore, is a $\delta$-ring by Lemma IX.6 As $\nu$ is $\sigma(\mathcal{K})$ compatible and $f$ is real, every $A \in \mathcal{Q}$ can be represented in the form $A=A_{+} \cup A_{-}$, where $A_{ \pm} \in \sigma(\mathcal{K})$ are such that $f(s) \geq 0$ for $\nu$-a.e. $s \in A_{+}$and $f(s) \leq 0$ for $\nu$-a.e. $s \in A_{-}$. Since $A_{ \pm}$are elements of $\sigma(\mathcal{Q})$ contained in an element of $\mathcal{Q}$, we conclude that $A_{ \pm} \in \mathcal{Q}$ and, therefore, $\int_{A_{ \pm}} f(s) d \nu(s)=0$. This implies that $f(s)=0$ for $\nu$-a.e. $s \in A$. Since $S_{\nu}$ is, up to a $\nu$-null set, a countable union of elements of $\mathcal{Q}$, we have $f(s)=0$ for $\nu$-a.e. $s$.

Proposition IX.13. Let $\nu$ be a $\sigma$-finite positive measure, $\Sigma$ be a countably generated $\sigma$-algebra, and $\mu$ be a $(\nu, \Sigma)$-measurable family of measures such that $\mu(s)$ is $\Sigma$-compatible for $\nu$-a.e. $s$. Then there is a unique (up to $\nu$-identity) $\nu$-measurable family $\mathfrak{S}$ of Hilbert spaces satisfying the conditions:

(1) $\mathfrak{S}(s)=L_{2}(G, \mu(s))$ for $\nu$-a.e. $s$, where $G$ is the largest set in $\Sigma$.

(2) $A \nu$-a.e. defined section $\xi$ of $\mathfrak{S}$ is $\nu$-measurable if and only if $s \rightarrow \int_{A} \xi(s \mid E) d \mu(s \mid E)$ is a $\nu$-measurable function for any $A \in \mathcal{Q}_{\mu, \nu}^{\Sigma}$.

Let $\mathcal{K} \subset \mathcal{Q}_{\mu, \nu}^{\Sigma}$ be closed under finite intersections and satisfy $\sigma(\mathcal{K})=\Sigma$. If $\xi$ is a $\nu$-a.e. defined section of $\mathfrak{S}$ such that $s \rightarrow \int_{A} \xi(s \mid E) d \mu(s \mid E)$ is a $\nu$-measurable function for any $A \in \mathcal{K}$, then $\xi$ is $\nu$-measurable.

Proof. Let $\mathcal{L}_{0}$ be a countable set generating $\Sigma$. Without loss of generality, we can assume that $\mathcal{L}_{0}$ is closed under finite intersections. Let $B_{1} \subset B_{2} \subset \ldots$ be a sequence of elements of $\mathcal{Q}_{\mu, \nu}^{\Sigma}$ such that $G=\bigcup_{i=1}^{\infty} B_{i}$ and let $\mathcal{L}$ be the set of all sets of the form $A \cap B_{i}$ for some $A \in \mathcal{L}_{0}$ and $i=1,2, \ldots$. Then $\mathcal{L}$ is a countable subset of $\mathcal{Q}_{\mu, \nu}^{\Sigma}$ that is closed under finite intersections and satisfies $\Sigma=\sigma(\mathcal{L})$. Let $\mathfrak{S}$ be $\nu$-a.e. defined family of Hilbert spaces satisfying (1). We choose a numbering $A_{1}, A_{2}, \ldots$ of the set $\mathcal{L}$ and endow $\mathfrak{S}$ with a $\nu$-measurable structure by setting

$$
\xi_{i}^{\mathfrak{S}}(s)=\left[\chi_{A_{i}}\right]_{\mu(s)}, \quad i=1,2, \ldots,
$$

for $\nu$-a.e. $s$, where the function $\chi_{A_{i}}$ is equal to unity on $A_{i}$ and vanishes on $G \backslash A_{i}$. Since $\left\langle\xi_{i}^{\mathfrak{S}}(s), \xi_{j}^{\mathfrak{S}}(s)\right\rangle=$ $\mu\left(s \mid A_{i} \cap A_{j}\right)$ and $\mathcal{Q}_{\mu, \nu}^{\Sigma}$ is a ring, $s \rightarrow\left\langle\xi_{i}^{\mathfrak{S}}(s), \xi_{j}^{\mathfrak{S}}(s)\right\rangle$ is a $\nu$-measurable function for any $i, j=1,2, \ldots$ If $\psi \in \mathfrak{S}(s)$ is orthogonal to $\xi_{i}^{\mathfrak{S}}(s)$ for all $i$, then Lemma IX.12 implies that $[\psi]_{\mu(s)}=0$. This means that the linear span of the sequence $\xi_{1}^{\mathfrak{S}}(s), \xi_{2}^{\mathfrak{S}}(s), \ldots$ is dense in $\mathfrak{S}(s)$ for $\nu$-a.e. $s$. Thus, $\mathfrak{S}$ endowed with the sequence $\xi_{1}^{\mathfrak{S}}, \xi_{2}^{\mathfrak{S}}, \ldots$ is indeed a $\nu$-measurable family of Hilbert spaces. If $\xi$ is a $\nu$-measurable section of $\mathfrak{S}$, then $s \rightarrow\left\langle\xi(s),\left[\chi_{A}\right]_{\mu(s)}\right\rangle=\int_{A} \xi(s \mid E) d \mu(s \mid E)$ is a $\nu$-measurable function for any $A \in \mathcal{L}$, and Lemma IX.2 implies that this is also true for every $A \in \mathcal{Q}_{\mu, \nu}^{\Sigma}$. Thus, $\mathfrak{S}$ satisfies (2). Suppose now that $\mathcal{K} \subset \mathcal{Q}_{\mu, \nu}^{\Sigma}$ is closed under finite intersections and satisfies $\sigma(\mathcal{K})=\Sigma$. By Lemma IX.2, if $\xi$ is a $\nu$-a.e. defined section of $\mathfrak{S}$ such that $s \rightarrow \int_{A} \xi(s \mid E) d \mu(s \mid E)$ is a $\nu$-measurable function for any $A \in \mathcal{K}$, then this is also true for any $A \in \mathcal{Q}_{\mu, \nu}^{\Sigma}$ and it follows from (2) that $\xi$ is $\nu$-measurable.

Proposition IX.14. Let $\nu, \mu, \Sigma$, and $\mathfrak{S}$ be as in Proposition IX.13, Let $\mathfrak{H}=\int{ }^{\oplus} \mathfrak{S}(s) d \nu(s)$ and $M=(\Sigma)-\int^{\oplus} \mu(s) d \nu(s)$. Given an $M$-measurable function $f$, let $\hat{f}$ denote the $\nu$-equivalence class such that $\hat{f}(s)$ is the $\mu(s)$-equivalence class of the map $E \rightarrow f(s, E)$ for $\nu$-a.e. $s$. Then the following statements hold:

1. $M$-measurable functions $f_{1}$ and $f_{2}$ are $M$-equivalent if and only if $\hat{f}_{1}=\hat{f}_{2}$.

2. If $f \in L_{2}\left(S_{M}, M\right)$, then $\hat{f} \in \mathfrak{H}$ and the operator $Q: L_{2}\left(S_{M}, M\right) \rightarrow \mathfrak{H}$ taking $f$ to $\hat{f}$ is unitary.

3. If $g$ is an $M$-measurable complex function, then $\hat{g}(s)$ is $\mu(s)$-measurable for $\nu$-a.e. $s, s \rightarrow \mathcal{T}_{\hat{g}(s)}^{\mu(s)}$ is a $\nu$-measurable family of operators in $\mathfrak{S}$, and

$$
Q \mathcal{T}_{g}^{M} Q^{-1}=\int^{\oplus} \mathcal{T}_{\hat{g}(s)}^{\mu(s)} d \nu(s)
$$

Proof. 1. Since $f_{1}$ and $f_{2}$ are $M$-measurable, the set $N=\left\{(s, E) \in S_{M}: f_{1}(s, E) \neq f_{2}(s, E)\right\}$ is $M$ measurable. By Lemma IX.9, $f_{1}$ and $f_{2}$ are $M$-equivalent if and only if $N_{s}$ is a $\mu(s)$-null set for $\nu$-a.e. $s$. Clearly, this condition holds if and only if $\hat{f}_{1}(s)=\hat{f}_{2}(s)$ for $\nu$-a.e. $s$ and, hence, $\hat{f}_{1}=\hat{f}_{2}$. 
2. Let $f \in L_{2}\left(S_{M}, M\right)$. By Corollary IX.11 $\hat{f}(s)$ is a $\mu(s)$-measurable function for $\nu$-a.e. $s$. Since $|f|^{2}$ is $M$-integrable, Proposition IX.10 implies that $|\hat{f}(s)|^{2}$ is $\mu(s)$-integrable and, therefore, $\hat{f}(s) \in \mathfrak{S}(s)$ for $\nu$-a.e. $s$. For every $A \in \mathcal{Q}_{\mu, \nu}^{\Sigma}$, the function $\chi_{A} \hat{f}(s)$, where $\chi_{A}$ is as in the proof of Proposition IX.13 is a product of two $\mu(s)$-square-integrable functions and, hence, is $\mu(s)$-integrable for $\nu$-a.e. $s$. Applying Corollary IX.11 to the function $(s, E) \rightarrow \chi_{A}(E) f(s, E)$, we conclude that $s \rightarrow \int_{A} \hat{f}(s \mid E) d \mu(s \mid E)$ is a $\nu$-measurable function for any $A \in \mathcal{Q}_{\mu, \nu}^{\Sigma}$. In view of Proposition [X.13, this means that $\hat{f}$ is a $\nu$ measurable section of $\mathfrak{S}$. By Proposition IX.10, $s \rightarrow\|\hat{f}(s)\|^{2}$ is a $\nu$-integrable function and we have $\|f\|^{2}=\int\|\hat{f}(s)\|^{2} d \nu(s)$. This means that $\hat{f} \in \mathfrak{H}$ and the operator $Q$ is isometric. We now prove that $Q$ is unitary. For this, it suffices to show that its image is dense in $\mathfrak{H}$. In other words, we have to show that every $\xi \in \mathfrak{H}$ that is orthogonal to $\hat{f}$ for every $f \in L_{2}\left(S_{M}, M\right)$ is equal to zero. Given $A \in \mathcal{Q}_{\mu, \nu}^{\Sigma}$, let $\mathcal{K}_{A}$ denote the set of all $B \in D_{\nu}$ such that $B \times A \in D_{M}$. It is clear that $\mathcal{K}_{A}$ is closed under finite intersections and $\sigma\left(\mathcal{K}_{A}\right)=\sigma\left(D_{\nu}\right)$. For a set $C \subset S_{M}$, let $X_{C}$ denote the function on $S_{M}$ that is equal to unity on $C$ and vanishes on $S_{M} \backslash C$. For $A \in \mathcal{Q}_{\mu, \nu}^{\Sigma}$ and $B \in \mathcal{K}_{A}$, the function $X_{B \times A}$ is obviously $M$-square-integrable and, therefore, we have

$$
\int_{B}\left\langle\left[\chi_{A}\right]_{\mu(s)}, \xi(s)\right\rangle d \nu(s)=\int\left\langle\hat{X}_{B \times A}(s), \xi(s)\right\rangle d \nu(s)=\left\langle\hat{X}_{B \times A}, \xi\right\rangle=0 .
$$

Applying Lemma IX.12 to $\mathcal{K}=\mathcal{K}_{A}$, we conclude that $\left\langle\left[\chi_{A}\right]_{\mu(s)}, \xi(s)\right\rangle=0$ for $\nu$-a.e. $s$ for every $A \in \mathcal{Q}_{\mu, \nu}^{\Sigma}$. Acting as in the proof of Proposition IX.13, we choose a sequence $A_{1}, A_{2}, \ldots$ of elements of $\mathcal{Q}_{\mu, \nu}^{\Sigma}$ such that the linear span of $\left[\chi_{A_{i}}\right]_{\mu(s)}$ is dense in $\mathfrak{S}(s)$ for $\nu$-a.e. $s$. Then, for $\nu$-a.e. $s, \xi(s)$ is orthogonal to all vectors $\left[\chi_{A_{i}}\right]_{\mu(s)}$ and, hence, is equal to zero. Thus, $\xi=0$ and the unitarity of $Q$ is proved.

3. By Corollary IX.11 $\hat{g}(s)$ is $\mu(s)$-measurable for $\nu$-a.e. $s$. Let $C_{1} \subset C_{2} \subset \ldots$ be a sequence of elements of $D_{M}$ such that $S_{M}=\bigcup_{j=1}^{\infty} C_{j}$ and $g$ is $M$-essentially bounded on $C_{j}$ for every $j=1,2, \ldots$ For $i, j=1,2, \ldots$, we define the function $h_{i j}$ on $S_{M}$ by setting $h_{i j}(s, E)=\chi_{A_{i}}(E) X_{C_{j}}(s, E)$, where $A_{i}$ are as in the proof of (2). It is clear that $h_{i j}$ is $M$-square-integrable and, therefore, $\hat{h}_{i j}$ is a $\nu$-measurable section of $\mathfrak{S}$ for all $i, j$. To prove the $\nu$-measurability of the family $s \rightarrow \mathcal{T}_{\hat{g}(s)}^{\mu(s)}$, it suffices to show that $\hat{h}_{i j}(s)$ belongs to the domain of $\mathcal{T}_{\hat{g}(s)}^{\mu(s)}$ and the linear span of the vectors $\left(\hat{h}_{i j}(s), \mathcal{T}_{\hat{g}(s)}^{\mu(s)} \hat{h}_{i j}(s)\right)$ is dense in the graph of $\mathcal{T}_{\hat{g}(s)}^{\mu(s)}$ for $\nu$-a.e. $s$. For $j=1,2, \ldots$, let $P_{j}$ be a $\nu$-a.e. defined map such that $P_{j}(s)=\mathcal{T}_{\chi C_{j, s}}^{\mu(s)}$ for $\nu$-a.e. $s$, where $C_{j, s}=\left\{E:(s, E) \in C_{j}\right\}$. Then, for $\nu$-a.e. $s, P_{j}(s)$ is an orthogonal projection commuting with $\mathcal{T}_{\hat{g}(s)}^{\mu(s)}$ and satisfying the equality

$$
\hat{h}_{i j}(s)=P_{j}(s)\left[\chi_{A_{i}}(s)\right]_{\mu(s)}
$$

for all $i, j$. In view of Lemma [X.9. $\hat{g}(s)$ is $\mu(s)$-essentially bounded on $C_{j, s}$ for $\nu$-a.e. $s$. Hence $\operatorname{Im} P_{j}(s)$ is contained in the domain of $\mathcal{T}_{\hat{g}(s)}^{\mu(s)}$ and $\mathcal{T}_{\hat{g}(s)}^{\mu(s)} P_{j}(s)$ is a bounded operator for $\nu$-a.e. $s$. In particular, it follows from (92) that $\hat{h}_{i j}(s)$ is in the domain of $\mathcal{T}_{\hat{g}(s)}^{\mu(s)}$ for $\nu$-a.e. $s$. Let $\mathcal{G}_{s}$ be the subset of the graph of $\mathcal{T}_{\hat{g}(s)}^{\mu(s)}$ consisting of all its elements $(\psi, \tilde{\psi})$ such that $\psi, \tilde{\psi} \in \operatorname{Im} P_{j}(s)$ for some $j=1,2, \ldots$. For $\nu$-a.e. $s$, we have $\lim _{j \rightarrow \infty} P_{j}(s) \psi=\psi$ for every $\psi \in \mathfrak{S}(s)$. As $P_{j}(s)$ commute with $\mathcal{T}_{\hat{g}(s)}^{\mu(s)}$, this implies that $\mathcal{G}_{s}$ is dense in the graph of $\mathcal{T}_{\hat{g}(s)}^{\mu(s)}$ for $\nu$-a.e. $s$. Given $(\psi, \tilde{\psi}) \in \mathcal{G}_{s}$ and $\varepsilon>0$, we can find a finite linear combination $\tau$ of vectors $\left[\chi_{A_{i}}\right]_{\mu(s)}$ such that $\|\psi-\tau\|<\varepsilon$. Then we have

$$
\begin{aligned}
& \left\|\psi-P_{j}(s) \tau\right\|=\left\|P_{j}(s)(\psi-\tau)\right\|<\varepsilon, \\
& \left\|\tilde{\psi}-\mathcal{T}_{\hat{g}(s)}^{\mu(s)} P_{j}(s) \tau\right\|=\left\|\mathcal{T}_{\hat{g}(s)}^{\mu(s)} P_{j}(s)(\psi-\tau)\right\|<\left\|\mathcal{T}_{\hat{g}(s)}^{\mu(s)} P_{j}(s)\right\| \varepsilon,
\end{aligned}
$$

where $j$ is such that $\psi, \tilde{\psi} \in \operatorname{Im} P_{j}(s)$. In view of (92), $P_{j}(s) \tau$ is a linear combination of $\hat{h}_{i j}(s)$, and it follows from the above inequalities that the linear span of the vectors $\left(\hat{h}_{i j}(s), \mathcal{T}_{\hat{g}(s)}^{\mu(s)} \hat{h}_{i j}(s)\right)$ is dense in $\mathcal{G}_{s}$ and, hence, in the graph of $\mathcal{T}_{\hat{g}(s)}^{\mu(s)}$ for $\nu$-a.e. $s$. The $\nu$-measurability of the family $s \rightarrow \mathcal{T}_{\hat{g}(s)}^{\mu(s)}$ is thus proved. Set $T=\int^{\oplus} \mathcal{T}_{\hat{g}(s)}^{\mu(s)} d \nu(s)$. Let $f$ belong to the domain of $\mathcal{T}_{g}^{M}$ and $F=\mathcal{T}_{g}^{M} f$. In view of Lemma IX.9, $\hat{F}(s)$ is $\mu(s)$-equivalent to $\hat{g}(s) \hat{f}(s)$ for $\nu$-a.e. $s$. This means that $Q f=\hat{f} \in D_{T}$ and $Q \mathcal{T}_{g}^{M} f=T Q f$. It follows that $T$ is an extension of $Q \mathcal{T}_{g}^{M} Q^{-1}$. To finish the proof, we have to show that $Q^{-1} \xi$ belongs to the domain of $\mathcal{T}_{g}^{M}$ for any $\xi \in D_{T}$. Let $\varphi=g Q^{-1} \xi$. For $\nu$-a.e. $s$, we have $(T \xi)(s \mid E)=\hat{g}(s \mid E) \xi(s \mid E)=\varphi(s, E)$ for $\mu(s)$-a.e. $E$. As $T \xi \in \mathfrak{H}$, it follows that the function $E \rightarrow \varphi(s, E)$ is $\mu(s)$-square-integrable for $\nu$-a.e. $s$ and the function $s \rightarrow \int|\varphi(s, E)|^{2} d \mu(s \mid E)$ is $\nu$-integrable. In view of Corollary IX.11, this implies that $\varphi$ is $M$-square-integrable, i.e., $Q^{-1} \xi$ is in the domain of $\mathcal{T}_{g}^{M}$. 


\section{EIGENFUNCTION EXPANSIONS}

In this section, we prove the results formulated in Sec. II

Let $\mathcal{B}_{\mathbb{R}}$ denote the Borel $\sigma$-algebra on $\mathbb{R}$. Proposition $\amalg$. 4 follows immediately from the next lemma.

Lemma X.1. Let $\phi \in \mathbb{R}$ and $\theta$ be a Borel real function on $A^{\phi}$. Then $\mu_{\theta}^{\phi}$ is a $\left(\nu_{0}, \mathcal{B}_{\mathbb{R}}\right)$-measurable family of measures and $M=\left(\mathcal{B}_{\mathbb{R}}\right)-\int^{\oplus} \mu_{\theta}^{\phi}(s) d \nu_{0}(s)$ is the unique Borel measure on $S \times \mathbb{R}$ satisfying the conditions of Proposition II.4.

Proof. Let $\mathcal{K}$ be the set of compact subsets of $\mathbb{R}$. Clearly, $\mathcal{K}$ is contained in the domain of $\mu_{\theta}^{\phi}(s)$ for all $s \in S$. Hence, the $\left(\nu_{0}, \mathcal{B}_{\mathbb{R}}\right)$-measurability of $\mu_{\theta}^{\phi}$ is ensured by Lemma I.3 and Corollary IX.3 (note that $\left.\sigma(\mathcal{K})=\mathcal{B}_{\mathbb{R}}\right)$

Let $\mathcal{B}_{S}$ and $\mathcal{B}_{S \times \mathbb{R}}$ denote the Borel $\sigma$-algebras of $S$ and $S \times \mathbb{R}$ respectively. By Definition IX.8. $M$ satisfies the conditions of Proposition IX.7 for $\mu=\mu_{\theta}^{\phi}, \nu=\nu_{0}, \Sigma=\mathcal{B}_{\mathbb{R}}$, and $\tilde{\Sigma}=\mathcal{B}_{S}$. By Lemma 【.3. we have $K^{\prime} \times K \in \Delta_{\mu, \nu}^{\Sigma}$ for any compact sets $K^{\prime} \subset S$ and $K \subset \mathbb{R}$. Since $\mathcal{B}_{S \times \mathbb{R}}=\sigma\left(\mathcal{B}_{S} \otimes \mathcal{B}_{\mathbb{R}}\right)$, Proposition IX.7 implies that $M$ is a Borel measure on $S \times \mathbb{R}$ satisfying (31). If $f$ is an $M$-measurable function, then equality (32) is ensured by Proposition IX.10. Thus, $M$ satisfies the conditions of Proposition II.4

Suppose $\tilde{M}$ is another Borel measure on $S$ satisfying (31). Let $\mathcal{L}$ be the set of all sets $A \in \mathcal{B}_{S \times \mathbb{R}} \cap$ $D_{M} \cap D_{\tilde{M}}$ such that $M(A)=\tilde{M}(A)$. Let $\tilde{\mathcal{K}}$ be the set of all sets $K^{\prime} \times K$, where $K^{\prime} \subset S$ and $K \subset \mathbb{R}$ are compact sets. Then $\tilde{\mathcal{K}}$ is closed under finite intersections and $\mathcal{L}$ is an $\alpha$-class containing $\tilde{\mathcal{K}}$. Since $\mathcal{L} \subset \sigma(\tilde{\mathcal{K}})=\mathcal{B}_{S \times \mathbb{R}}$, Lemma IX.6 implies that $\mathcal{L}$ is a $\delta$-ring. We hence have $M=\tilde{M}$ by Lemma A.3.

Let $\phi \in \mathbb{R}$ and $\theta$ be a Borel real function on $A^{\phi}$. We define the operator-valued map $\mathcal{U}_{\theta}^{\phi}$ on $S$ by setting

$$
\mathcal{U}_{\theta}^{\phi}(s)=\left\{\begin{array}{cc}
U_{m+\phi}, & s \in S \backslash A^{\phi}, \\
U_{m+\phi, \theta(s)}, & s \in A^{\phi},
\end{array}\right.
$$

for all $s=(m, p) \in S$, where the operators $U_{\kappa}$ and $U_{\kappa, \vartheta}$ satisfy the conditions of Proposition VI.3 and Proposition VI.4 respectively. By Lemma X.1, the assumptions of Proposition IX.13 are fulfilled for $\Sigma=\mathcal{B}_{\mathbb{R}}, \mu=\mu_{\theta}^{\phi}$, and $\nu=\nu_{0}$. A $\nu_{0}$-measurable family $\mathfrak{S}$ of Hilbert spaces satisfying the conditions of Proposition IX.13 for $\operatorname{such} \Sigma, \mu$, and $\nu$ will be denoted by $\mathfrak{S}_{\theta}^{\phi}$.

Lemma X.2. Let $\phi \in \mathbb{R}, \theta$ be a Borel real function on $A^{\phi}$, and $\psi \in L_{2}^{c}\left(\mathbb{R}_{+}\right)$. Then $s \rightarrow \mathcal{U}_{\theta}^{\phi}(s) \psi$ is a $\nu_{0}$-measurable section of $\mathfrak{S}_{\theta}^{\phi}$.

Proof. As follows from Proposition IX.13 applied to the set $\mathcal{K}$ of all compact subsets of $\mathbb{R}$, it suffices to show that

$$
I_{K}(s)=\int_{K}\left(\mathcal{U}_{\theta}^{\phi}(s) \psi\right)(E) d \mu_{\theta}^{\phi}(s \mid E)
$$

is a Borel function on $S$ for any compact set $K \subset \mathbb{R}$. Given $-1<\kappa<1$, let the function $f_{K}^{\kappa}$ on $\mathbb{R}$ be defined by the relation

$$
f_{K}^{\kappa}(\vartheta)=\cos \left(\vartheta-\vartheta_{\kappa}\right) \int_{K} F_{\kappa}^{(1)}(E) d \mathcal{V}_{\kappa, \vartheta}(E)+\sin \left(\vartheta-\vartheta_{\kappa}\right) \int_{K} F_{\kappa}^{(2)}(E) d \mathcal{V}_{\kappa, \vartheta}(E)
$$

where $\vartheta_{\kappa}$ is defined by (17) and the continuous functions $F_{\kappa}^{(1)}$ and $F_{\kappa}^{(2)}$ on $\mathbb{R}$ are given by

$$
F_{\kappa}^{(1)}(E)=\int_{0}^{\infty} u^{\kappa}(E \mid r) \psi(r) d r, \quad F_{\kappa}^{(2)}(E)=\int_{0}^{\infty} w^{\kappa}(E \mid r) \psi(r) .
$$

It follows from (30), (57), (62), and (93) that $I_{K}(s)=f_{K}^{m+\phi}(\theta(s))$ for every $s=(m, p) \in A^{\phi}$. On the other hand, $p \rightarrow I_{K}(m, p)$ is a constant function on $\mathbb{R}$ for every $m$ satisfying $|m+\phi| \geq 1$. Since $f_{K}^{\kappa}$ is a Borel function on $\mathbb{R}$ by Lemma II.2 we conclude that $I_{K}$ is a Borel function on $S$.

In what follows, we set $\mathfrak{h}=L_{2}\left(\mathbb{R}_{+}\right)$.

Corollary X.3. Let $\phi \in \mathbb{R}$ and $\theta$ be a Borel real function on $A^{\phi}$. Then $\mathcal{U}_{\theta}^{\phi}$ is a $\nu_{0}$-measurable family of operators from $\mathcal{I}_{\mathfrak{h}, \nu_{0}}$ to $\mathfrak{S}_{\theta}^{\phi}$.

Proof. The statement follows immediately from Lemma X.2 and Lemma B.3. 
Given $\phi \in \mathbb{R}$ and a Borel real function $\theta$ on $A^{\phi}$, we define the linear operator $U_{\theta}^{\phi}$ from $L_{2}\left(S, \mathfrak{h}, \nu_{0}\right)$ to $\int^{\oplus} \mathfrak{S}_{\theta}^{\phi}(s) d \nu_{0}(s)$ by setting

$$
U_{\theta}^{\phi}=\int^{\oplus} \mathcal{U}_{\theta}^{\phi}(s) d \nu_{0}(s)
$$

Since $\mathcal{U}_{\theta}^{\phi}(s)$ is a unitary operator from $\mathfrak{h}$ to $\mathfrak{S}_{\theta}^{\phi}(s)$ for $\nu_{0}$-a.e. $s$, it follows from Corollary X.3 and Proposition B.23 that $U_{\theta}^{\phi}$ is a unitary operator. If $f$ is an $M_{\theta}^{\phi}$-measurable complex function, we denote by $\left.f\right|_{\theta} ^{\phi}$ the $\nu_{0}$-equivalence class such that $\left.f\right|_{\theta} ^{\phi}(s)$ is the $\mu_{\theta}^{\phi}(s)$-equivalence class of the map $E \rightarrow f(s, E)$ for $\nu_{0}$-a.e. $s$. In view of Lemma X.1, all conditions of Proposition IX.14 are fulfilled for $\Sigma=\mathcal{B}_{\mathbb{R}}, \nu=\nu_{0}$, $\mu=\mu_{\theta}^{\phi}, M=M_{\theta}^{\phi}, \mathfrak{S}=\mathfrak{S}_{\theta}^{\phi}$, and $\hat{f}=\left.f\right|_{\theta} ^{\phi}$. It follows from statement 2 of Proposition IX.14 that the correspondence $\left.f \rightarrow f\right|_{\theta} ^{\phi}$ induces a unitary operator $Q_{\theta}^{\phi}$ from $L_{2}\left(S \times \mathbb{R}, M_{\theta}^{\phi}\right)$ to $\int^{\oplus} \mathfrak{S}_{\theta}^{\phi}(s) d \nu_{0}(s)$.

Note that the uniqueness of $W$ in Proposition $\llbracket .1$ is ensured by the density of $L_{2}^{c}\left(\mathbb{R}^{3}\right)$ in $L_{2}\left(R^{3}\right)$. Hence, Proposition II.1 follows from the next lemma.

Lemma X.4. Let $\phi \in \mathbb{R}, \theta$ be a Borel real function on $A^{\phi}$, and $V$ be as in Lemma VIII.1. Then the operator $W=\left(Q_{\theta}^{\phi}\right)^{-1} U_{\theta}^{\phi} V$ satisfies the conditions of Proposition II.1.

Proof. Let $\Phi \in C_{0}^{\infty}(\mathscr{O})$ and $\Psi=[\Phi]_{\Lambda}$. For $s \in S$ and $E \in \mathbb{R}$, let $h_{\Psi}(s, E)$ denote the right-hand side of (10). For $-1<\kappa<1$ and $m \in \mathbb{Z}$, we define the continuous function $F_{m}^{\kappa}$ on $\mathbb{R} \times \mathbb{R} \times(\mathscr{O})$ by the relation

$$
F_{m}^{\kappa}(E, \vartheta, x)=\frac{e^{-i p x_{3}}}{2 \pi \sqrt{r_{x}}}\left(\frac{x_{1}-i x_{2}}{r_{x}}\right)^{m} u_{\vartheta}^{\kappa}\left(E \mid r_{x}\right)
$$

for all $E, \vartheta \in \mathbb{R}$ and $x=\left(x_{1}, x_{2}, x_{3}\right) \in \mathscr{O}$, where $r_{x}=\sqrt{x_{1}^{2}+x_{2}^{2}}$. Set $f_{m, \Psi}^{\kappa}(E, \vartheta)=\int F_{m}^{\kappa}(E, \vartheta, x) \Psi(x) d x$. By (11), (19), and (10), we have $h_{\Psi}(s, E)=f_{m, \Psi}^{m+\phi}(E, \theta(s))$ for all $s=(m, p) \in A^{\phi}$ and $E \in \mathbb{R}$. If $|m+\phi|>1$, then (11), (19), and (10) imply that $(p, E) \rightarrow h_{\Psi}(m, p ; E)$ is a continuous function on $\mathbb{R}^{2}$. Since $f_{m, \Psi}^{\kappa}$ is a continuous function on $\mathbb{R}^{2}$ for all $m \in \mathbb{Z}$ and $-1<\kappa<1$, it follows that $(p, E) \rightarrow h_{\Psi}(m, p ; E)$ is a Borel function on $\mathbb{R}^{2}$ for every $m \in \mathbb{Z}$. Thus, $h_{\Psi}$ is a Borel (and, hence, $M_{\theta}^{\phi}$-measurable) function on $S \times \mathbb{R}$.

In view of (11), passing to the polar coordinates in the $\left(x_{1}, x_{2}\right)$-plane in the integral in (10) yields

$$
h_{\Psi}(s, E)=\int_{0}^{\infty} \mathcal{J}_{\theta}^{\phi}(s, E \mid r) \tilde{\Phi}(s \mid r) d r
$$

for all $s \in S$ and $E \in \mathbb{R}$, where the $C_{0}^{\infty}\left(\mathbb{R}_{+}\right)$-valued map $\tilde{\Phi}$ on $S$ is given by (71). It follows from (19), (30), (93), Proposition VI.3, and Proposition VI.4 that, for every $s \in S$ and $\psi \in L_{2}^{c}\left(\mathbb{R}_{+}\right)$, the equality

$$
\left(\mathcal{U}_{\theta}^{\phi}(s) \psi\right)(E)=\int_{0}^{\infty} \mathcal{J}_{\theta}^{\phi}(s, E \mid r) \psi(r) d r
$$

holds for $\mu_{\theta}^{\phi}(s)$-a.e. $E$. In view of (72), equalities (94), (95) and (96) imply that the function $E \rightarrow h_{\Psi}(s, E)$ is $\mu_{\theta}^{\phi}(s)$-equivalent to $\left(U_{\theta}^{\phi} V \Psi\right)(s)$ for $\nu_{0}$-a.e. $s$, whence $U_{\theta}^{\phi} V \Psi=\left.h_{\Psi}\right|_{\theta} ^{\phi}$. Since $U_{\theta}^{\phi} V \Psi=Q_{\theta}^{\phi} W \Psi=\left.(W \Psi)\right|_{\theta} ^{\phi}$, it follows from statement 1 of Proposition IX.14 that $W \Psi$ is $M_{\theta}^{\phi}$-equivalent to $h_{\Psi}$. Thus, (10) holds for $\Psi=[\Phi]_{\Lambda}$ with $\Phi \in C_{0}^{\infty}(\mathscr{O})$.

For a general $\Psi \in L_{2}^{c}\left(\mathbb{R}^{3}\right)$, we choose a sequence $\Phi_{n} \in C_{0}^{\infty}(\mathscr{O})$ such that $\Psi_{n}=\left[\Phi_{n}\right]_{\Lambda}$ converge to $\Psi$ in $L_{2}\left(\mathbb{R}^{3}\right)$ and the supports of all $\Phi_{n}$ are contained in a fixed compact set for all $n$. Since $\mathcal{W}_{\theta}^{\phi}(s, E)$ is locally square-integrable, we have $h_{\Psi_{n}}(s, E) \rightarrow h_{\Psi}(s, E)$ for all $s \in S$ and $E \in \mathbb{R}$. As $W \Psi_{n} \rightarrow W \Psi$ in $L_{2}\left(S \times \mathbb{R}, M_{\theta}^{\phi}\right)$ by continuity of $W$, it follows that $W \Psi$ is $M_{\theta}^{\phi}$-equivalent to $h_{\Psi}$.

Lemma X.4 and the uniqueness statement of Proposition II.1 imply that

$$
W_{\theta}^{\phi}=\left(Q_{\theta}^{\phi}\right)^{-1} U_{\theta}^{\phi} V
$$

Theorem II.5 follows from Proposition VIII.5 and the next statement.

Proposition X.5. Let $\phi \in \mathbb{R}, \theta$ be a Borel real function on $A^{\phi}$, and $R_{\theta}^{\phi}$ be defined by (87). Then we have $H_{\theta}^{\phi}=R_{\theta}^{\phi}$. 
Proof. For brevity, we set $\mu=\mu_{\theta}^{\phi}, \mathcal{U}=\mathcal{U}_{\theta}^{\phi}, U=U_{\theta}^{\phi}, Q=Q_{\theta}^{\phi}, M=M_{\theta}^{\phi}$, and $\mathfrak{g}=\left.\mathfrak{f}\right|_{\theta} ^{\phi}$. It follows from (30), (33), (78), (82), (93), and Propositions VI.3 and VI.4 that

$$
\mathcal{U}(s) \mathcal{H}_{\theta}^{\phi}(s) \mathcal{U}(s)^{-1}=\mathcal{T}_{\mathfrak{g}(s)}^{\mu(s)}
$$

for $\nu_{0}$-a.e. s. By Lemma VIII.4. $\mathcal{H}_{\theta}^{\phi}$ is a $\nu_{0}$-measurable family of operators in $\mathfrak{h}$. Hence, it follows from (87), (94), Corollary X.3, statement 1 of Proposition B.23, and Proposition B.25 that $s \rightarrow \mathcal{T}_{\mathfrak{g}(s)}^{\mu(s)}$ is a $\nu_{0}$-measurable family of operators in $\mathfrak{S}_{\theta}^{\phi}$ and

$$
U V R_{\theta}^{\phi}(U V)^{-1}=\int^{\oplus} \mathcal{T}_{\mathfrak{g}(s)}^{\mu(s)} d \nu_{0}(s) .
$$

By statement 3 of Proposition IX.14, the operator in the right-hand side is equal to $Q \mathcal{T}_{\mathfrak{f}}^{M} Q^{-1}$. Equalities (34) and (97) therefore imply that $H_{\theta}^{\phi}=R_{\theta}^{\phi}$.

\section{ACKNOWLEDGMENTS}

The author is grateful to I.V. Tyutin and B.L. Voronov for useful discussions.

\section{Appendix A: Measure theory}

In this Appendix, we briefly describe the measure-theoretic concepts used in this paper. Our aim here is not only to fix the notation but also to formulate definitions in such a way as to provide a unified treatment of positive and spectral measures. In particular, in contrast to the standard approach (see, e.g., Refs. 34 and 36 ), we define positive measures as functions on $\delta$-rings rather than $\sigma$-algebras and do not allow them to take infinite values (see Sec. A2 below).

\section{Rings and algebras of sets}

Recall that a nonempty set of sets $\mathcal{Q}$ is called a ring of sets if $A \cup B \in \mathcal{Q}$ and $A \backslash B \in \mathcal{Q}$ for any $A, B \in \mathcal{Q}$. As $A \cap B=A \backslash(A \backslash B)$, every ring is closed under finite intersections. A ring $\mathcal{Q}$ is called a $\sigma$-ring (a $\delta$-ring) if it is closed under countable unions (resp., under countable intersections). For a set of sets $\mathcal{Q}$, we denote by $\sigma(\mathcal{Q})(\delta(\mathcal{Q}))$ the $\sigma$-ring (resp., $\delta$-ring) generated by $\mathcal{Q}$, i.e., the smallest $\sigma$-ring (resp., $\delta$-ring) containing $\mathcal{Q}$. If $\mathcal{Q}$ is a $\delta$-ring, then $\sigma(\mathcal{Q})$ is just the set of all countable unions of elements of $\mathcal{Q}$. A $\sigma$-ring $\mathcal{Q}$ is said to be countably generated if there is a countable set $\tilde{\mathcal{Q}} \subset \mathcal{Q}$ such that $\mathcal{Q}=\sigma(\tilde{\mathcal{Q}})$. A $\sigma$-ring $\mathcal{Q}$ is called a $\sigma$-algebra if it has the largest element with respect to inclusion.

The $\sigma$-ring generated by all open sets of a topological space $X$ is obviously a $\sigma$-algebra. It is called the Borel $\sigma$-algebra of $X$ and its elements are called Borel subsets of $X$. A map $f$ is called a Borel map from $X$ to a topological space $Y$ if $X$ is contained in the domain $D_{f}$ of $f, f(X) \subset Y$, and $f^{-1}(A) \cap X$ is a Borel subset of $X$ for any Borel subset $A$ of $Y$.

\section{Measures}

Let $\mathfrak{A}$ be a topological Abelian group and $\nu$ be an $\mathfrak{A}$-valued map. Let $\mathcal{N}_{\nu}$ denote the set of all $N \in D_{\nu}$ with the property: if $N^{\prime} \subset N$ and $N^{\prime} \in D_{\nu}$, then $\nu\left(N^{\prime}\right)=0$. We say that $\nu$ is an $\mathfrak{A}$-valued $\sigma$-additive function if $\nu(A)=\sum_{i \in I} \nu\left(A_{i}\right)$ for any $A \in D_{\nu}$ and any countable partition $A=\bigcup_{i \in I} A_{i}$ with $A_{i} \in D_{\nu}$. An $\mathfrak{A}$-valued $\sigma$-additive function is called an $\mathfrak{A}$-valued measure if its domain $D_{\nu}$ is a $\delta$-ring and the following completeness conditions are satisfied:

(a) If $A \in \sigma\left(D_{\nu}\right)$ and the family $\left\{\nu\left(A_{i}\right)\right\}_{i \in I}$ is summable in $\mathfrak{A}$ for any countable partition $A=\bigcup_{i \in I} A_{i}$ with $A_{i} \in D_{\nu}$, then $A \in D_{\nu}$.

(b) If $N \in \mathcal{N}_{\nu}$ and $N^{\prime} \subset N$, then $N^{\prime} \in D_{\nu}$ (and, hence, $N^{\prime} \in \mathcal{N}_{\nu}$ ).

Elements of $\mathcal{N}_{\nu}$ are called $\nu$-null sets and elements of $\sigma\left(D_{\nu}\right)$ are called $\nu$-measurable sets. A measure $\nu$ is called $\sigma$-finite if $\sigma\left(D_{\nu}\right)$ is a $\sigma$-algebra. In this case, we denote by $S_{\nu}$ the largest element of $\sigma\left(D_{\nu}\right)$ with respect to inclusion. A measure $\nu$ is called finite if it is $\sigma$-finite and $S_{\nu} \in D_{\nu}$. A measure $\nu$ is called positive if it is $\mathbb{R}$-valued and $\nu(A) \geq 0$ for any $A \in D_{\nu}$. 
Remark A.1. In most expositions of measure theory (see, e.g., Refs 34 and 36), a positive measure is defined as a $\sigma$-additive function on a $\sigma$-algebra taking values in the extended real semi-axis $\overline{\mathbb{R}}_{+}=[0, \infty]$. Such an $\overline{\mathbb{R}}_{+}$-valued measure $\nu$ is called $\sigma$-finite if the largest set in $D_{\nu}$ is a countable union of elements of $D_{\nu}$ with a finite measure. It is called complete if every subset of an element of $D_{\nu}$ with zero measure also belongs to $D_{\nu}$. Complete $\sigma$-finite $\overline{\mathbb{R}}_{+}$-valued measures can be naturally identified with positive $\sigma$-finite measures in our sense (note that a complete $\sigma$-finite $\overline{\mathbb{R}}_{+}$-valued measure $\nu$ restricted to the set of all elements of $D_{\nu}$ with a finite measure is a positive $\sigma$-finite measure in our sense). The advantage of our definition is that it does not involve the extended real axis and, therefore, makes it possible to treat positive measures in the same way as vector-valued measures.

Given a $\sigma$-ring $\mathcal{Q}$, a measure $\nu$ is called $\mathcal{Q}$-compatible if $\mathcal{Q} \subset \sigma\left(D_{\nu}\right)$ and for every $A \in D_{\nu}$, there is an $N \in \mathcal{N}_{\nu}$ such that $A \cup N \in \mathcal{Q}$. In this case, $D_{\nu}$ consists of all sets of the form $B \cup N$, where $B \in \mathcal{Q} \cap D_{\nu}$ and $N \in \mathcal{N}_{\nu}$. If $X$ is a topological space and $\mathcal{B}$ is its Borel $\sigma$-algebra, then a $\mathcal{B}$-compatible measure is called a Borel measure on $X$.

Remark A.2. Positive Borel measures on a topological space $X$ are usually defined as $\overline{\mathbb{R}}_{+}$-valued $\sigma$-additive functions on the Borel $\sigma$-algebra of $X$. If such a measure is $\sigma$-finite, then its completion corresponds (as described in Remark A.1 to a positive Borel measure on $X$ in our sense. Since we consider only complete measures (see condition (b) above), we introduce the notion of $\mathcal{Q}$-compatibility to relate measure and topology.

Lemma A.3. Let $\mathfrak{A}$ be a sequentially complete topological Abelian group and $\nu$ be an $\mathfrak{A}$-valued $\sigma$-additive function such that $D_{\nu}$ is a $\delta$-ring. Then there is a unique $\sigma\left(D_{\nu}\right)$-compatible $\mathfrak{A}$-valued measure $\hat{\nu}$ such that $D_{\nu} \subset D_{\hat{\nu}}$ and $\hat{\nu}$ coincides with $\nu$ on $D_{\nu}$. If $\nu$ satisfies (a), then $D_{\nu}=D_{\hat{\nu}} \cap \sigma\left(D_{\nu}\right)$.

The measure $\hat{\nu}$ is called the completion of the $\sigma$-additive function $\nu$.

Proof. We give the proof only for positive $\nu$ because the lemma is used in this paper only in this case. The existence and uniqueness of $\hat{\nu}$ is then guaranteed by the well-known results on the extension of positive measures. Let $\nu$ satisfy (a) and $A \in D_{\hat{\nu}} \cap \sigma\left(D_{\nu}\right)$. Let $\left\{A_{i}\right\}_{i \in I}$ be a countable partition of $A$ such that $A_{i} \in D_{\nu}$ for all $i \in I$. Since $\hat{\nu}\left(A_{i}\right)=\nu\left(A_{i}\right)$ for all $i \in I$, the $\sigma$-additivity of $\hat{\nu}$ implies that the family $\left\{\nu\left(A_{i}\right)\right\}_{i \in I}$ is summable. Condition (a) hence ensures that $A \in D_{\nu}$ and, therefore, $D_{\nu} \supset D_{\hat{\nu}} \cap \sigma\left(D_{\nu}\right)$. As the opposite inclusion also obviously holds, the lemma is proved.

Let $\nu$ be an $\mathfrak{A}$-valued measure and $A$ be a $\nu$-measurable set. The restriction $\left.\nu\right|_{A}$ of $\nu$ to $A$ is, by definition, the restriction of the map $\nu$ to the domain $D_{\left.\nu\right|_{A}}$ consisting of all elements of $D_{\nu}$ that are contained in $A$. Clearly, $\left.\nu\right|_{A}$ is a $\sigma$-finite measure for any $\nu$-measurable set $A$ and $S_{\left.\nu\right|_{A}}=A$.

Let $\nu_{1}$ and $\nu_{2}$ be positive measures and $\mathcal{Q}$ be the $\sigma$-ring generated by all sets of the form $A_{1} \times A_{2}$, where $A_{1} \in D_{\nu_{1}}$ and $A_{2} \in D_{\nu_{2}}$. Then there is a unique positive $\mathcal{Q}$-compatible measure $\nu$ such that $\nu\left(A_{1} \times A_{2}\right)=\nu_{1}\left(A_{1}\right) \nu_{2}\left(A_{2}\right)$ for every $A_{1} \in D_{\nu_{1}}$ and $A_{2} \in D_{\nu_{2}}$. This measure is called the product of $\nu_{1}$ and $\nu_{2}$ and is denoted by $\nu_{1} \times \nu_{2}$.

\section{Standard measures}

Let $\mathfrak{A}$ be a topological Abelian group. An $\mathfrak{A}$-valued measure $\nu$ is called standard if it is $\sigma$-finite and there exists a complete separable metric space $X$ such that $X \subset S_{\nu}, S_{\nu} \backslash X$ is a $\nu$-null set, and $\left.\nu\right|_{X}$ is a Borel measure on $X$. If $\nu$ is a standard measure and $A$ is a $\nu$-measurable set, then $\left.\nu\right|_{A}$ is also a standard measure.

Remark A.4. Standard measures were first introduced in Ref. 37. Unlike Ref. 37, we consider only complete measures, and standard measures in our sense are actually the completions of those in the sense of Ref. 37. In the probabilistic context, such complete measures were introduced in Ref. 38 under the name of Lebesgue measure spaces. The class of standard measures is broad enough to include most measures encountered in applications and, at the same time, is narrow enough to exclude measures with a pathological behavior.

\section{Measurable maps}

Let $\nu$ be a $\sigma$-finite $\mathfrak{A}$-valued measure. A map $f$ is said to be defined $\nu$-a.e. if $S_{\nu} \backslash D_{f}$ is a $\nu$-null set. Given a set $X$, a map $f$ is said to be an $X$-valued $\nu$-a.e. defined map if $S_{\nu} \backslash f^{-1}(X)$ is a $\nu$-null set. If $X$ is a topological space, then a map $f$ is called an $X$-valued $\nu$-measurable map if $f$ is an $X$-valued $\nu$-a.e. 
defined map and $f^{-1}(B) \cap S_{\nu}$ is a $\nu$-measurable set for any Borel subset $B$ of $X$. We say that some property $P(s)$ holds for $\nu$-a.e. $s$, if there is a $\nu$-null set $N$ such that $P(s)$ holds for all $s \in S_{\nu} \backslash N$.

Given a $\nu$-measurable set $A$, we say that $f$ is an $X$-valued $\nu$-measurable map on $A$ if $f$ is an $X$-valued $\left.\nu\right|_{A}$-measurable map. A property $P(s)$ is said to hold for $\nu$-a.e. $s \in A$ if it holds for $\left.\nu\right|_{A}$-a.e. $s$.

A complex $\nu$-a.e. defined function $f$ is said to be $\nu$-essentially bounded if there is $C>0$ such that $|f(s)| \leq C$ for $\nu$-a.e. $s$.

Remark A.5. Note that the domain $D_{f}$ of a $\nu$-a.e. defined or $\nu$-measurable map $f$ is not assumed to be contained in $S_{\nu}$. Moreover, $X$-valued $\nu$-a.e. defined (and, in particular, $\nu$-measurable) maps are allowed to take values outside $X$ on some $\nu$-null set contained in $S_{\nu} \cap D_{f}$. This implies in particular, that using "the set of all $X$-valued $\nu$-measurable maps" would lead to the same kind of set-theoretic problems as the use of "the set of all sets".

Let $X$ be a complete separable metric space, $f$ be an $X$-valued $\nu$-a.e. defined map, and $f_{1}, f_{2}, \ldots$ be a sequence of $X$-valued $\nu$-measurable maps such that $f_{n}(s)$ converge to $f(s)$ in $X$ as $n \rightarrow \infty$ for $\nu$-a.e. $s$. Then $f$ is $\nu$-measurable.

\section{Equivalence classes}

Let $\nu$ be a $\sigma$-finite $\mathfrak{A}$-valued measure. Two $\nu$-a.e. defined maps $f$ and $g$ are called $\nu$-equivalent if $f(s)=g(s)$ for $\nu$-a.e. $s$. All $\nu$-a.e. defined maps fall into disjoint classes of $\nu$-equivalent maps, which are called $\nu$-equivalence classes. In every $\nu$-equivalence class, we choose an arbitrary fixed element whose domain of definition is contained in $S_{\nu}$. Given a $\nu$-a.e. defined map $f$, we denote by $[f]_{\nu}$ such a chosen element belonging to the $\nu$-equivalence class containing $f$. Thus, the map $[f]_{\nu}$ is $\nu$-equivalent to $f$ for any $\nu$-a.e. defined map $f$, and we have $[f]_{\nu}=[g]_{\nu}$ for every pair of $\nu$-equivalent maps $f$ and $g$. If $X$ is a topological space, then $f$ is an $X$-valued $\nu$-measurable map if and only if so is $[f]_{\nu}$.

In this paper, $\nu$-equivalence classes per se are not used. Whenever we speak of $\nu$-equivalence classes, we always refer to representatives of the form $[f]_{\nu}$, where $f$ is a $\nu$-a.e. defined map. There are two reasons for such a redefinition of the notion of a $\nu$-equivalence class. First, the same arguments as in Remark A.5 show that the $\nu$-equivalence classes in the true sense cannot be considered as well-defined sets. Hence, using them as elements of sets is not satisfactory from the viewpoint of foundations of mathematics. Choosing a fixed representative in each class allows us to circumvent this difficulty. Second, since $\nu$ equivalence classes in our sense are just some maps, all definitions and notations introduced for maps become directly applicable to $\nu$-equivalence classes.

\section{Integrable functions}

Let $\nu$ be a $\sigma$-finite positive measure. A $\nu$-measurable complex function $f$ is called $\nu$-integrable if

$$
I_{\nu}(f)=\sup _{A_{i} \in D_{\nu}, c_{i} \in \mathbb{R}} \sum_{i=1}^{n} c_{i} \nu\left(A_{i}\right)<\infty,
$$

where the supremum is taken over all finite sets $A_{1}, \ldots, A_{n}$ of disjoint elements of $D_{\nu}$ and $c_{1}, \ldots, c_{n} \in \mathbb{R}$ such that $c_{i} \leq|f(s)|$ for $\nu$-a.e. $s \in A_{i}$. The integral $\int f(s) d \nu(s)$ of a $\nu$-integrable function $f$ is a complex number that is uniquely determined by the conditions that $\int f(s) d \nu(s)$ be linear in $f$ and coincide with $I_{\nu}(f)$ if $f(s) \geq 0$ for $\nu$-a.e. $s$.

Clearly, passing to a $\nu$-equivalent function does not affect its $\nu$-integrability. In particular, a $\nu$-a.e. defined function $f$ is $\nu$-integrable if and only if $[f]_{\nu}$ is $\nu$-integrable. If $A$ is a $\nu$-measurable set and $f$ is a $\left.\nu\right|_{A}$-integrable function, then we say that $f$ is a $\nu$-integrable function on $A$. In this case, we write $\int_{A} f(s) d \nu(s)$ in place of $\left.\int f(s) d \nu\right|_{A}(s)$.

Remark A.6. As for $\nu$-measurable functions, the domain of a $\nu$-integrable function is not assumed to be contained in $S_{\nu}$.

\section{7. $L_{2}$-spaces}

Let $\nu$ be a $\sigma$-finite positive measure and $\mathfrak{h}$ be a separable Hilbert space .39 We denote by $\mathcal{M}(\mathfrak{h}, \nu)$ the set of all elements $[f]_{\nu}$, where $f$ is an $\mathfrak{H}$-valued $\nu$-measurable map. The set $\mathcal{M}(\mathfrak{h}, \nu)$ has a natural structure of a vector space over $\mathbb{C}$ (for any $\mathfrak{h}$-valued $\nu$-a.e. defined maps $f$ and $g$ and any $k \in \mathbb{C}$, we set 
$[f]_{\nu}+[g]_{\nu}=[f+g]_{\nu}$ and $\left.k[f]_{\nu}=[k f]_{\nu}\right)$. Given a $\nu$-measurable set $A$, we denote by $L_{2}(A, \mathfrak{h}, \nu)$ the linear subspace of $\mathcal{M}\left(\mathfrak{h},\left.\nu\right|_{A}\right)$ consisting of all its elements $f$ such that $s \rightarrow\|f(s)\|^{2}$ is a $\nu$-integrable function. Defining the scalar product of $f, g \in L_{2}(A, \mathfrak{h}, \nu)$ by the formula

$$
\langle f, g\rangle=\int_{A}\langle f(s), g(s)\rangle d \nu(s)
$$

we make $L_{2}(A, \mathfrak{h}, \nu)$ into a Hilbert space.

For $\mathfrak{h}=\mathbb{C}$, we denote the space $L_{2}(A, \mathfrak{h}, \nu)$ by $L_{2}(A, \nu)$. If $A \subset \mathbb{R}^{n}$ and $\nu$ is the Lebesgue measure on $\mathbb{R}^{n}$, the space $L_{2}(A, \nu)$ is denoted by $L_{2}(A)$.

Given a $\nu$-measurable complex function $g$, we denote by $\mathcal{T}_{g}^{\nu}$ the operator of multiplication by $g$ in $L_{2}\left(S_{\nu}, \nu\right)$. By definition, the graph of $\mathcal{T}_{g}^{\nu}$ consists of all pairs $\left(f_{1}, f_{2}\right)$ of elements of $L_{2}\left(S_{\nu}, \nu\right)$ such that $f_{2}(s)=g(s) f_{1}(s)$ for $\nu$-a.e. $s$. The operator $\mathcal{T}_{g}^{\nu}$ is closed and densely defined and its adjoint is equal to $\mathcal{T}_{\bar{g}}^{\nu}$, where $\bar{g}$ is the complex conjugate function of $g$. In particular, if $g$ is real, then $\mathcal{T}_{g}^{\nu}$ is self-adjoint.

\section{Spectral measures}

We refer the reader to Chapter 5 of Ref. 40 for a detailed exposition of the theory of spectral measures. Here, we only give a brief summary of the facts needed in this paper. Let $\mathfrak{H}$ be a separable Hilbert space and $L(\mathfrak{H})$ be the space of bounded everywhere defined linear operators in $\mathfrak{H}$ endowed with the strong operator topology. A finite $L(\mathfrak{H})$-valued measure $\mathcal{E}$ is called a spectral measure in $\mathfrak{H}$ if $\mathcal{E}(A)$ is an orthogonal projection in $\mathfrak{H}$ for any $A \in D_{\mathcal{E}}$ and $\mathcal{E}\left(S_{\mathcal{E}}\right)$ is the identity operator in $\mathfrak{H}$. For any $\Psi \in \mathfrak{H}$, we define the positive measure $\mathcal{E}_{\Psi}$ as the completion of the positive $\sigma$-additive function $A \rightarrow\langle\mathcal{E}(A) \Psi, \Psi\rangle$ on $D_{\mathcal{E}}$, where $\langle\cdot, \cdot\rangle$ is the scalar product on $\mathfrak{H}$.

Let $\mathcal{E}$ be a spectral measure. Given an $\mathcal{E}$-measurable complex function $f$, the integral $J_{f}^{\mathcal{E}}$ of $f$ with respect to $\mathcal{E}$ is defined as the unique linear operator in $\mathfrak{H}$ such that

$$
\begin{gathered}
D_{J_{f}^{\mathcal{E}}}=\left\{\Psi \in \mathfrak{H}: \int|f(s)|^{2} d \mathcal{E}_{\Psi}(s)<\infty\right\} \\
\left\langle\Psi, J_{f}^{\mathcal{E}} \Psi\right\rangle=\int f(s) d \mathcal{E}_{\Psi}(s), \quad \Psi \in D_{J_{f}^{\mathcal{E}}} .
\end{gathered}
$$

For any $\mathcal{E}$-measurable complex function $f$, the operator $J_{f}^{\mathcal{E}}$ is closed and densely defined and its adjoint is equal to $J_{\bar{f}}$.

For every normal operator $T$, there is a unique Borel spectral measure $\mathcal{E}_{T}$ on $\mathbb{C}$ such that $J_{g}^{\mathcal{E}_{T}}=T$, where $g$ is the identity function on $\mathbb{C}$. The operators $\mathcal{E}_{T}(A)$, where $A$ is a Borel subset of $\mathbb{C}$, are called the spectral projections of $T$. If $f$ is an $\mathcal{E}_{T}$-measurable complex function, then the operator $J_{f}^{\mathcal{E}_{T}}$ is also denoted as $f(T)$.

\section{Appendix B: Direct integral decompositions of operators in Hilbert space}

One of the main mathematical tools used in this paper are the direct integral decompositions of operators in Hilbert space. In its original form ${ }^{6}$, the theory of such decompositions (also known as von Neumann's reduction theory) is applicable only to bounded operators ${ }^{41}$ and, therefore, is insufficient for quantum-mechanical applications, where unbounded operators play a prominent role. An extension of the reduction theory to unbounded operators was given in Ref. 7 (most results of Ref. 7 were earlier formulated without proofs in Ref. 8). The approach proposed in Ref. 7 is based on the observation ${ }^{42}$ that the properties of a closed operator in a Hilbert space can be encoded in a set of four bounded operators forming its so called characteristic matrix. This allows one to reformulate problems concerning the direct integrals of arbitrary closed operators in terms of direct integrals of bounded operators. Although this approach makes it possible to extend the results of the original von Neumann's theory to unbounded operators, it seems to be somewhat inconvenient for applications to concrete problems, where the computation of the characteristic matrices may turn out to be a difficult task. In particular, the definition of the measurability of families of operators given in Ref. 7 may be difficult to verify for concrete examples. From the viewpoint of applications, the definition of measurability proposed in Ref. 8 and formulated directly in terms of the graphs of operators seems to be more suitable (both definitions are actually equivalent, see Remark B.12 below).

In this appendix, we give a self-contained exposition of the theory of direct integral decompositions of (generally, unbounded) operators in Hilbert space. Our treatment is based on the formulation of 
measurability proposed in Ref. 8 and does not involve characteristic matrices. Such an approach allows us to derive all results in a straightforward manner: unlike Ref. 7, we do not need the theory for bounded operators as a prerequisite. Moreover, it becomes possible to give a concise treatment of direct integral decompositions of sums and products of operators (see Propositions B.25 and B.26 below), whose original analysis in Ref. 43 involves lengthy computations of characteristic matrices.

In what follows, we use the measure-theoretic framework described in Appendix A Throughout this appendix, $\nu$ denotes a $\sigma$-finite positive measure.

\section{Measurable families of Hilbert spaces}

A $\nu$-a.e. defined map $\mathfrak{S}$ is called a $\nu$-a.e. defined family of Hilbert spaces if $\mathfrak{S}(s)$ is a separable Hilbert space for $\nu$-a.e. $s$. A map $\xi$ is called a $\nu$-a.e. defined section of $\mathfrak{S}$ if $\xi(s) \in \mathfrak{S}(s)$ for $\nu$-a.e. $s$.

A $\nu$-a.e. defined family of Hilbert spaces $\mathfrak{S}$ is called $\nu$-measurable if it is equipped with a sequence $\left\{\xi_{i}^{\mathfrak{S}}\right\}_{i=1}^{\infty}$ of $\nu$-a.e. defined sections of $\mathfrak{S}$ such that the linear span of the vectors $\xi_{1}^{\mathfrak{S}}(s), \xi_{2}^{\mathfrak{S}}(s), \ldots$ is dense in $\mathfrak{S}(s)$ for $\nu$-a.e. $s$, and $s \rightarrow\left\langle\xi_{i}^{\mathfrak{S}}(s), \xi_{j}^{\mathfrak{S}}(s)\right\rangle$ is a $\nu$-measurable complex function for any $i, j=1,2, \ldots$ A $\nu$-a.e. defined section $\xi$ of $\mathfrak{S}$ is called $\nu$-measurable if $s \rightarrow\left\langle\xi_{i}^{\mathfrak{S}}(s), \xi(s)\right\rangle$ is a $\nu$-measurable complex function for any $i=1,2, \ldots$.

We say that $\nu$-measurable families $\mathfrak{S}_{1}$ and $\mathfrak{S}_{2}$ of Hilbert spaces are $\nu$-identical if they are $\nu$-equivalent and have the same $\nu$-measurable sections.

A sequence $e_{1}, e_{2}, \ldots$ of elements of a Hilbert space $\mathfrak{H}$ is said to be a generalized orthonormal system in $\mathfrak{H}$ if $\left\langle e_{i}, e_{j}\right\rangle=0$ for $i \neq j$ and $\left\|e_{i}\right\|$ is equal to either 1 or 0 for any $i=1,2, \ldots$.

Let $\mathfrak{S}$ be a $\nu$-a.e. defined family of Hilbert spaces and $\xi_{1}, \xi_{2}, \ldots$ be a sequence of $\nu$-a.e. defined sections of $\mathfrak{S}$. We say that $\nu$-a.e. defined sections $\tilde{\xi}_{1}, \tilde{\xi}_{2}, \ldots$ of $\mathfrak{S}$ constitute an orthonormal sequence associated with $\xi_{1}, \xi_{2}, \ldots$ if the following conditions hold

(a) For $\nu$-a.e. $s$, the sequence $\tilde{\xi}_{1}(s), \tilde{\xi}_{2}(s), \ldots$ is a generalized orthonormal system in $\mathfrak{S}(s)$ whose linear span coincides with that of $\xi_{1}(s), \xi_{2}(s), \ldots$

(b) There exist $\nu$-measurable complex functions $f_{i j}$ defined for $i \leq j$ such that $\tilde{\xi}_{j}(s)=\sum_{i=1}^{j} f_{i j}(s) \xi_{i}(s)$ for all $j=1,2, \ldots$ and $\nu$-a.e. $s$.

Lemma B.1. Let $\mathfrak{S}$ be a $\nu$-a.e. defined family of Hilbert spaces and $\xi_{1}, \xi_{2}, \ldots$ be a sequence of $\nu$-a.e. defined sections of $\mathfrak{S}$ such that $s \rightarrow\left\langle\xi_{i}(s), \xi_{j}(s)\right\rangle$ is a $\nu$-measurable complex function for any $i, j=1,2, \ldots$ Then there exists an orthonormal sequence associated with $\xi_{1}, \xi_{2}, \ldots$

Proof. The required sequence is constructed by applying the standard orthogonalization procedure to $\xi_{1}(s), \xi_{2}(s), \ldots$, see the proof of Lemma 1 in Sec. II.1.2 of Ref. 19 for details.

If $\xi$ and $\eta$ are $\nu$-measurable sections of a $\nu$-measurable family $\mathfrak{S}$ of Hilbert spaces, then $s \rightarrow\langle\xi(s), \eta(s)\rangle$ is a $\nu$-measurable complex function. Indeed, by Lemma B.1 there exists an orthonormal sequence $\tilde{\xi}_{1}, \tilde{\xi}_{2}, \ldots$ associated with $\xi_{1}^{\mathfrak{S}}, \xi_{2}^{\mathfrak{S}}, \ldots$ By $(\mathrm{a})$, the linear span of $\tilde{\xi}_{1}(s), \tilde{\xi}_{2}(s), \ldots$ is dense in $\mathfrak{S}(s)$ for $\nu$-a.e. $s$. This implies that $\langle\xi(s), \eta(s)\rangle=\sum_{i=1}^{\infty}\left\langle\xi(s), \tilde{\xi}_{i}(s)\right\rangle\left\langle\tilde{\xi}_{i}(s), \eta(s)\right\rangle$ for $\nu$-a.e. $s$. Hence the required statement follows because $s \rightarrow\left\langle\xi(s), \tilde{\xi}_{i}(s)\right\rangle$ and $s \rightarrow\left\langle\tilde{\xi}_{i}(s), \eta(s)\right\rangle$ are $\nu$-measurable complex functions by (b) and the $\nu$-measurability of $\xi$ and $\eta$.

Lemma B.2. Let $\mathfrak{S}$ be a $\nu$-measurable family of Hilbert spaces and $\xi_{1}, \xi_{2}, \ldots$ be $\nu$-measurable sections of $\mathfrak{S}$. Let $\xi$ be a $\nu$-a.e. defined map such that $\xi(s)$ belongs to the closed linear span of $\xi_{1}(s), \xi_{2}(s), \ldots$ for $\nu$-a.e. s. If $s \rightarrow\left\langle\xi_{j}(s), \xi(s)\right\rangle$ is a $\nu$-measurable function for all $j=1,2, \ldots$, then $\xi$ is a $\nu$-measurable section of $\mathfrak{S}$.

Proof. By Lemma B.1 there exists an orthonormal sequence $\tilde{\xi}_{1}, \tilde{\xi}_{2}, \ldots$ associated with $\xi_{1}, \xi_{2}, \ldots$ By condition (b), $\tilde{\xi}_{j}$ is a $\nu$-measurable section of $\mathfrak{S}$ for every $j=1,2, \ldots$ By condition (a), $\tilde{\xi}_{1}(s), \tilde{\xi}_{2}(s), \ldots$ is a generalized orthonormal system whose closed linear span coincides with that of $\xi_{1}(s), \xi_{2}(s), \ldots$ and, hence, contains $\xi(s)$ for $\nu$-a.e. $s$. This implies that $\xi(s)=\sum_{j=1}^{\infty}\left\langle\tilde{\xi}_{j}(s), \xi(s)\right\rangle \tilde{\xi}_{j}(s)$ for $\nu$-a.e. $s$ and, therefore, $\xi$ is a $\nu$-measurable section of $\mathfrak{S}$.

\section{Direct sums of measurable families}

Let $\mathfrak{S}_{1}$ and $\mathfrak{S}_{2}$ be $\nu$-measurable families of Hilbert spaces. Then there is a unique (up to $\nu$-identity) $\nu$-measurable family $\mathfrak{S}$ of Hilbert spaces satisfying the conditions 
(1) $\mathfrak{S}(s)=\mathfrak{S}_{1}(s) \oplus \mathfrak{S}_{2}(s)$ for $\nu$-a.e. $s$.

(2) A $\nu$-a.e. defined map $\zeta$ is a $\nu$-measurable section of $\mathfrak{S}$ if and only if there are $\nu$-measurable sections $\xi$ and $\eta$ of $\mathfrak{S}_{1}$ and $\mathfrak{S}_{2}$ respectively such that $\zeta(s)=(\xi(s), \eta(s))$ for $\nu$-a.e. $s$.

Indeed, every $\nu$-a.e. defined map $\mathfrak{S}$ satisfying (1) becomes a $\nu$-measurable family of Hilbert spaces satisfying (2) if we choose $\xi_{1}^{\mathfrak{S}}, \xi_{2}^{\mathfrak{S}}, \ldots$ in such a way that $\xi_{2 k}^{\mathfrak{S}}(s)=\left(\xi_{k}^{\mathfrak{S}_{1}}(s), 0\right)$ and $\xi_{2 k-1}^{\mathfrak{S}}(s)=\left(0, \xi_{k}^{\mathfrak{S}_{2}}(s)\right)$ for $\nu$-a.e. $s$ and every $k=1,2, \ldots$ The $\nu$-measurable family $\mathfrak{S}$ of Hilbert spaces satisfying $(1)$ and $(2)$ is called the direct sum of $\mathfrak{S}_{1}$ and $\mathfrak{S}_{2}$ and is denoted by $\mathfrak{S}_{1} \oplus_{\nu} \mathfrak{S}_{2}$.

\section{Constant and discrete families}

Let $\mathfrak{h}$ be a separable Hilbert space. Then there exists a unique (up to $\nu$-identity) $\nu$-measurable family $\mathfrak{S}$ of Hilbert spaces satisfying the conditions

(1) $\mathfrak{S}(s)=\mathfrak{h}$ for $\nu$-a.e. $s$.

(2) A $\nu$-a.e. defined map $\xi$ is a $\nu$-measurable section of $\mathfrak{S}$ if and only if $\xi$ is an $\mathfrak{h}$-valued $\nu$-measurable map.

Indeed, let $e_{1}, e_{2}, \ldots$ be an orthonormal basis in $\mathfrak{h}$ and $\mathfrak{S}$ be a $\nu$-a.e. defined map satisfying (1). To make $\mathfrak{S}$ into a $\nu$-measurable family, we require $\xi_{1}^{\mathfrak{S}}, \xi_{2}^{\mathfrak{S}}, \ldots$ to be $\nu$-a.e. defined maps such that $\xi_{i}^{\mathfrak{S}}(s)=e_{i}$ for $\nu$-a.e. $s$. If $\xi$ is a $\nu$-measurable section of $\mathfrak{S}$, then $\xi$ is an $\mathfrak{H}$-valued $\nu$-measurable map because $\xi(s)=\sum_{i}\left\langle\xi_{i}^{\mathfrak{S}}(s), \xi(s)\right\rangle e_{i}$ for $\nu$-a.e. $s$ and, therefore, $\mathfrak{S}$ satisfies $(2)$. The $\nu$-measurable family $\mathfrak{S}$ of Hilbert spaces satisfying $(1)$ and $(2)$ is denoted by $\mathcal{I}_{\mathfrak{h}, \nu}$.

Suppose now that $\mathscr{S}$ is a countable set, $\nu$ is a counting measure on $\mathscr{S}$ and $\mathfrak{S}$ is a $\nu$-a.e. defined family of Hilbert spaces (i.e., $\mathscr{S} \subset D_{\mathfrak{S}}$ and $\mathfrak{S}(s)$ is a separable Hilbert space for every $s \in \mathscr{S}$ ). Since every $\nu$-a.e. defined complex function is $\nu$-measurable, there is a unique (up to $\nu$-identity) $\nu$-measurable family $\mathfrak{S}_{\mathscr{S}}$ of Hilbert spaces such that $\mathfrak{S}_{\mathscr{S}}(s)=\mathfrak{S}(s)$ for every $s \in \mathscr{S}$. Every $\nu$-a.e. defined section of $\mathfrak{S}_{\mathscr{S}}$ is $\nu$-measurable.

\section{Measurable families of operators}

Let $\mathfrak{S}$ be a $\nu$-measurable family of Hilbert spaces. A $\nu$-a.e. defined map $\mathfrak{S}^{\prime}$ is said to be a $\nu$-a.e. defined family of subspaces of $\mathfrak{S}$ if $\mathfrak{S}^{\prime}(s)$ is a linear (not necessarily closed) subspace of $\mathfrak{S}(s)$ for $\nu$-a.e. s. A $\nu$-a.e. defined family $\mathfrak{S}^{\prime}$ of subspaces of $\mathfrak{S}$ is called $\nu$-measurable if there is a sequence $\xi_{1}, \xi_{2}, \ldots$ of $\nu$-measurable sections of $\mathfrak{S}$ such that the linear span of $\xi_{1}(s), \xi_{2}(s), \ldots$ is dense in $\mathfrak{S}^{\prime}(s)$ for $\nu$-a.e. $s$ (such a sequence will be called a $\nu$-measurable basis in $\mathfrak{S}^{\prime}$ ).

Let $\mathfrak{S}_{1}$ and $\mathfrak{S}_{2}$ be $\nu$-measurable families of Hilbert spaces. A $\nu$-a.e. defined map $\mathcal{R}$ is called a $\nu$-a.e. defined family of operators from $\mathfrak{S}_{1}$ to $\mathfrak{S}_{2}$ if $\mathcal{R}(s)$ is an operator (possibly not everywhere defined and unbounded) from $\mathfrak{S}_{1}(s)$ to $\mathfrak{S}_{2}(s)$ for $\nu$-a.e. $s$. A $\nu$-a.e. defined family $\mathcal{R}$ of operators from $\mathfrak{S}_{1}$ to $\mathfrak{S}_{2}$ is called $\nu$-measurable if there are sequences $\xi_{1}, \xi_{2}, \ldots$ and $\eta_{1}, \eta_{2}, \ldots$ of $\nu$-measurable sections of $\mathfrak{S}_{1}$ and $\mathfrak{S}_{2}$ respectively such that the linear span of the vectors $\left(\xi_{j}(s), \eta_{j}(s)\right)$ is dense in the graph $G_{\mathcal{R}(s)}$ of the operator $\mathcal{R}(s)$ for $\nu$-a.e. $s$. In other words, $\mathcal{R}$ is $\nu$-measurable if $s \rightarrow G_{\mathcal{R}(s)}$ is a $\nu$-measurable family of subspaces of $\mathfrak{S}_{1} \oplus_{\nu} \mathfrak{S}_{2}$.

Given a $\nu$-measurable family $\mathfrak{S}$ of Hilbert spaces, we say that $\mathcal{R}$ is a $\nu$-a.e. defined $(\nu$-measurable) family of operators in $\mathfrak{S}$ if it is a $\nu$-a.e. defined (resp., $\nu$-measurable) family of operators from $\mathfrak{S}$ to $\mathfrak{S}$. If $\mathfrak{h}$ is a separable Hilbert space, then $\nu$-a.e. defined ( $\nu$-measurable) families of operators in $\mathcal{I}_{\mathfrak{h}, \nu}$ are called $\nu$-a.e. defined (resp., $\nu$-measurable) families of operators in $\mathfrak{h}$.

Lemma B.3. Let $\mathfrak{S}_{1}$ and $\mathfrak{S}_{2}$ be $\nu$-measurable families of Hilbert spaces and $\mathcal{R}$ be a $\nu$-a.e. defined family of operators from $\mathfrak{S}_{1}$ to $\mathfrak{S}_{2}$ such that $\mathcal{R}(s)$ is an everywhere defined bounded operator for $\nu$-a.e. s. Then the following statements hold

1. If $\mathcal{R}$ is $\nu$-measurable then $s \rightarrow \mathcal{R}(s) \xi(s)$ is a $\nu$-measurable section of $\mathfrak{S}_{2}$ for any $\nu$-measurable section $\xi$ of $\mathfrak{S}_{1}$.

2. Suppose there exists a $\nu$-measurable basis $\xi_{1}, \xi_{2}, \ldots$ in $\mathfrak{S}_{1}$ such that $s \rightarrow \mathcal{R}(s) \xi_{j}(s)$ is a $\nu$-measurable section of $\mathfrak{S}_{2}$ for all $j=1,2, \ldots$ Then $\mathcal{R}$ is $\nu$-measurable. 
Proof. 1. Let $\xi$ be a $\nu$-measurable section of $\mathfrak{S}_{1}$ and $\xi_{1}, \xi_{2}, \ldots$ and $\eta_{1}, \eta_{2}, \ldots$ be $\nu$-measurable sections of $\mathfrak{S}_{1}$ and $\mathfrak{S}_{2}$ respectively such that the linear span of the vectors $\left(\xi_{j}(s), \eta_{j}(s)\right)$ is dense in $G_{\mathcal{R}(s)}$ for $\nu$-a.e. $s$. By Lemma B.1, there exists an orthonormal sequence $\tilde{\xi}_{1}, \tilde{\xi}_{2}, \ldots$ associated with $\xi_{1}, \xi_{2}, \ldots$ By condition (b) of Sec. B1, we have $\mathcal{R}(s) \tilde{\xi}_{j}(s)=\sum_{i=1}^{j} f_{i j}(s) \eta_{i}(s)$ with some $\nu$-measurable functions $f_{i j}$ for any $j=1,2, \ldots$ and $\nu$-a.e. $s$. Hence, $s \rightarrow \mathcal{R}(s) \tilde{\xi}_{j}(s)$ is a $\nu$-measurable section of $\mathfrak{S}_{2}$ for any $j=1,2, \ldots$ By condition (a) of Sec. B1, the linear span of $\tilde{\xi}_{1}(s), \tilde{\xi}_{2}(s), \ldots$ coincides with that of $\xi_{1}(s), \xi_{2}(s), \ldots$ and, therefore, is dense in $\mathfrak{S}_{1}(s)$ for $\nu$-a.e. $s$. This implies that $\xi(s)=\sum_{j=1}^{\infty}\left\langle\tilde{\xi}_{j}(s), \xi(s)\right\rangle \tilde{\xi}_{j}(s)$ for $\nu$-a.e. $s$. By the continuity of $\mathcal{R}(s)$, it follows that $\mathcal{R}(s) \xi(s)=\sum_{j=1}^{\infty}\left\langle\tilde{\xi}_{j}(s), \xi(s)\right\rangle \mathcal{R}(s) \tilde{\xi}_{j}(s)$ for $\nu$-a.e. $s$ and, therefore, $s \rightarrow \mathcal{R}(s) \xi(s)$ is a $\nu$-measurable section of $\mathfrak{S}_{2}$.

2. Let $\nu$-measurable sections $\eta_{1}, \eta_{2}, \ldots$ of $\mathfrak{S}_{2}$ be such that $\eta_{j}(s)=\mathcal{R}(s) \xi_{j}(s)$ for all $j=1,2, \ldots$ and $\nu$-a.e. $s$. As the linear span of $\xi_{1}(s), \xi_{2}(s), \ldots$ is dense in $\mathfrak{S}_{1}(s)$ and $\mathcal{R}(s)$ is everywhere defined and continuous, the linear span of $\left(\xi_{j}(s), \eta_{j}(s)\right)$ is dense in $G_{\mathcal{R}(s)}$ for $\nu$-a.e. $s$. This means that $\mathcal{R}$ is $\nu$-measurable.

Lemma B.4. Let $\mathfrak{S}_{1}$ and $\mathfrak{S}_{2}$ be $\nu$-measurable families of Hilbert spaces and $\mathcal{R}$ be a $\nu$-measurable family of operators from $\mathfrak{S}_{1}$ to $\mathfrak{S}_{2}$ such that $\mathcal{R}(s)$ is an everywhere defined bounded operator for $\nu$-a.e. $s$. Let $\mathfrak{S}_{1}^{\prime}$ be a $\nu$-measurable family of subspaces of $\mathfrak{S}_{1}$. Then the images of $\mathfrak{S}_{1}^{\prime}(s)$ under $\mathcal{R}(s)$ constitute a $\nu$-measurable family of subspaces of $\mathfrak{S}_{2}$.

Proof. Let $\xi_{1}, \xi_{2}, \ldots$ be a $\nu$-measurable basis in $\mathfrak{S}_{1}^{\prime}$. By the continuity of $\mathcal{R}(s)$, the linear span of $\mathcal{R}(s) \xi_{1}(s), \mathcal{R}(s) \xi_{2}(s), \ldots$ is dense in the image of $\mathfrak{S}_{1}^{\prime}(s)$ under $\mathcal{R}(s)$ for $\nu$-a.e. $s$. Hence the statement follows because $s \rightarrow \mathcal{R}(s) \xi_{j}(s)$ is a $\nu$-measurable section of $\mathfrak{S}_{2}$ for any $j=1,2, \ldots$ by Lemma B.3.

Lemma B.5. Let $\mathfrak{S}$ be a $\nu$-measurable family of Hilbert spaces and $\mathfrak{S}^{\prime}$ and $\mathcal{P}$ be $\nu$-a.e. defined maps such that $\mathfrak{S}^{\prime}(s)$ is a closed subspace of $\mathfrak{S}(s)$ and $\mathcal{P}(s)$ is the orthogonal projection of $\mathfrak{S}(s)$ onto $\mathfrak{S}^{\prime}(s)$ for $\nu$-a.e. $s$. Then $\mathcal{P}$ is a $\nu$-measurable family of operators in $\mathfrak{S}$ if and only if $\mathfrak{S}^{\prime}$ is a $\nu$-measurable family of subspaces of $\mathfrak{S}$.

Proof. If $\mathcal{P}$ is $\nu$-measurable, then so is $\mathfrak{S}^{\prime}$ by Lemma B.4 Let $\mathfrak{S}^{\prime}$ be $\nu$-measurable and $\xi_{1}, \xi_{2}, \ldots$ be a $\nu$-measurable basis in $\mathfrak{S}^{\prime}$. Let $\xi$ be a $\nu$-measurable section of $\mathfrak{S}$. For $\nu$-a.e. $s$, we have $\left\langle\mathcal{P}(s) \xi(s), \xi_{j}(s)\right\rangle=$ $\left\langle\xi(s), \xi_{j}(s)\right\rangle$. Hence, $s \rightarrow\left\langle\mathcal{P}(s) \xi(s), \xi_{j}(s)\right\rangle$ is a $\nu$-measurable section of $\mathfrak{S}$ for all $j=1,2, \ldots$ In view of Lemma B.2, this implies that $s \rightarrow \mathcal{P}(s) \xi(s)$ is a $\nu$-measurable section of $\mathfrak{S}$ and, therefore, $\mathcal{P}$ is $\nu$-measurable by Lemma B.3.

Let $\mathfrak{H}^{\prime}$ and $\mathfrak{H}^{\prime \prime}$ be closed subspaces of a Hilbert space $\mathfrak{H}$ and $P^{\prime}$ and $P^{\prime \prime}$ be the orthogonal projections of $\mathfrak{H}$ onto $\mathfrak{H}^{\prime}$ and $\mathfrak{H}^{\prime \prime}$ respectively. Then we have $\mathfrak{H}^{\prime} \cap \mathfrak{H}^{\prime \prime}=\operatorname{Ker} R$, where $R=1_{\mathfrak{H}}-\left(P^{\prime}+P^{\prime \prime}\right) / 2$ and $1_{\mathfrak{H}}$ denotes the identity operator in $\mathfrak{H}$. Indeed, suppose $\psi \in \operatorname{Ker} R$ and $\psi \notin \mathfrak{H}^{\prime} \cap \mathfrak{H}^{\prime \prime}$. Assume, for definiteness, that $\psi \notin \mathfrak{H}^{\prime}$. Then $\left\|P^{\prime} \psi\right\|<\|\psi\|$. Since $\psi=\left(P^{\prime} \psi+P^{\prime \prime} \psi\right) / 2$ and $\left\|P^{\prime \prime} \psi\right\| \leq\|\psi\|$, this implies that $\|\psi\|<\|\psi\|$. We thus obtain a contradiction and the statement is proved.

Lemma B.6. Let $\mathfrak{S}$ be a $\nu$-measurable family of Hilbert spaces and $\mathfrak{S}^{\prime}$ and $\mathfrak{S}^{\prime \prime}$ be $\nu$-measurable families of closed subspaces of $\mathfrak{S}$. Then

1. If $\mathfrak{S}^{\prime \prime}(s) \subset \mathfrak{S}^{\prime}(s)$ for $\nu$-a.e. $s$, then $s \rightarrow \mathfrak{S}^{\prime}(s) \ominus \mathfrak{S}^{\prime \prime}(s)$, where $\mathfrak{S}^{\prime}(s) \ominus \mathfrak{S}^{\prime \prime}(s)$ is the orthogonal complement of $\mathfrak{S}^{\prime \prime}(s)$ in $\mathfrak{S}^{\prime}(s)$, is a $\nu$-measurable family of subspaces of $\mathfrak{S}$.

2. $s \rightarrow \mathfrak{S}^{\prime}(s) \cap \mathfrak{S}^{\prime \prime}(s)$ is a $\nu$-measurable family of subspaces of $\mathfrak{S}$.

Proof. 1. Let $\mathcal{P}^{\prime}$ and $\mathcal{P}^{\prime \prime}$ be $\nu$-a.e. defined maps such that $\mathcal{P}^{\prime}(s)$ and $\mathcal{P}^{\prime \prime}(s)$ are orthogonal projections of $\mathfrak{S}(s)$ onto $\mathfrak{S}^{\prime}(s)$ and $\mathfrak{S}^{\prime \prime}(s)$ respectively for $\nu$-a.e. $s$. By Lemma B.5. $\mathcal{P}^{\prime}$ and $\mathcal{P}^{\prime \prime}$ are $\nu$-measurable families of operators in $\mathfrak{S}$. It follows from Lemma B.3 that $s \rightarrow \mathcal{P}^{\prime}(s)-\mathcal{P}^{\prime \prime}(s)$ is also a $\nu$-measurable family of operators in $\mathfrak{S}$. As $\mathcal{P}^{\prime}(s)-\mathcal{P}^{\prime \prime}(s)$ is the orthogonal projection of $\mathfrak{S}(s)$ onto $\mathfrak{S}^{\prime}(s) \ominus \mathfrak{S}^{\prime \prime}(s)$ for $\nu$-a.e. $s$, the desired statement follows from Lemma B.5

2. Let $\nu$-measurable families $\mathcal{P}^{\prime}$ and $\mathcal{P}^{\prime \prime}$ of operators in $\mathfrak{S}$ be as in the proof of (1) and $\mathcal{R}$ be a $\nu$-a.e. defined map such that $\mathcal{R}(s)=1_{\mathfrak{S}(s)}-\left(\mathcal{P}^{\prime}(s)+\mathcal{P}^{\prime \prime}(s)\right) / 2$ for $\nu$-a.e. $s$. It follows from Lemma B.3 that $\mathcal{R}$ is a $\nu$-measurable family of operators in $\mathfrak{S}$. Since $\mathfrak{S}^{\prime}(s) \cap \mathfrak{S}^{\prime \prime}(s)=\operatorname{Ker} \mathcal{R}(s)=(\operatorname{Im} \mathcal{R}(s))^{\perp}$ for $\nu$-a.e. $s$, the statement follows from Lemma B.4 and (1).

Lemma B.7. Let $\mathfrak{S}_{1}$ and $\mathfrak{S}_{2}$ be $\nu$-measurable families of Hilbert spaces and $\mathcal{R}$ be a $\nu$-measurable family of operators from $\mathfrak{S}_{1}$ to $\mathfrak{S}_{2}$. Then

1. If $\mathcal{R}(s)$ is invertible for $\nu$-a.e. $s$, then $s \rightarrow \mathcal{R}(s)^{-1}$ is a $\nu$-measurable family of operators from $\mathfrak{S}_{2}$ to $\mathfrak{S}_{1}$. 
2. If $\mathcal{R}(s)$ is closed for $\nu$-a.e. $s$, then $s \rightarrow \operatorname{Ker} \mathcal{R}(s)$ is a $\nu$-measurable family of subspaces of $\mathfrak{S}_{1}$.

Proof. For $\nu$-a.e. $s$, let $\mathcal{S}(s)$ be everywhere defined bounded operator from $\mathfrak{S}_{1}(s) \oplus \mathfrak{S}_{2}(s)$ to $\mathfrak{S}_{2}(s) \oplus \mathfrak{S}_{1}(s)$ taking $\left(\psi_{1}, \psi_{2}\right)$ to $\left(\psi_{2}, \psi_{1}\right)$. Obviously, $\mathcal{S}$ is a $\nu$-measurable family of operators from $\mathfrak{S}_{1} \oplus_{\nu} \mathfrak{S}_{2}$ to $\mathfrak{S}_{2} \oplus_{\nu} \mathfrak{S}_{1}$. If $\mathcal{R}(s)$ is invertible for $\nu$-a.e. $s$, then $G_{\mathcal{R}(s)^{-1}}$ coincides with the image of $G_{\mathcal{R}(s)}$ under $\mathcal{S}(s)$ for $\nu$-a.e. $s$ and, therefore, (1) follows from Lemma B.4. Suppose now that $\mathcal{R}(s)$ is closed for $\nu$-a.e. $s$. Since $s \rightarrow \mathfrak{S}_{1}(s) \times\{0\}$ is a $\nu$-measurable family of closed subspaces of $\mathfrak{S}_{1} \oplus_{\nu} \mathfrak{S}_{2}$ and $G_{\mathcal{R}(s)} \cap\left(\mathfrak{S}_{1}(s) \times\{0\}\right)=$ $\operatorname{Ker} \mathcal{R}(s) \times\{0\}$ for $\nu$-a.e. $s$, statement 2 of Lemma B.6 implies that $s \rightarrow \operatorname{Ker} \mathcal{R}(s) \times\{0\}$ is a $\nu$-measurable family of closed subspaces of $\mathfrak{S}_{1} \oplus_{\nu} \mathfrak{S}_{2}$, whence (2) obviously follows.

Lemma B.8. Let $\mathfrak{S}_{1}, \mathfrak{S}_{2}$, and $\mathfrak{S}_{3}$ be $\nu$-measurable families of Hilbert spaces and $\mathcal{R}_{1}$ and $\mathcal{R}_{2}$ be $\nu$ measurable families of closed operators from $\mathfrak{S}_{1}$ to $\mathfrak{S}_{2}$ and from $\mathfrak{S}_{2}$ to $\mathfrak{S}_{3}$ respectively. Then $s \rightarrow$ $\mathcal{R}_{2}(s) \mathcal{R}_{1}(s)$ is a $\nu$-measurable family of operators from $\mathfrak{S}_{1}$ to $\mathfrak{S}_{3}$.

Proof. Let $\mathfrak{S}^{\prime}$ and $\mathfrak{S}^{\prime \prime}$ be $\nu$-a.e. defined maps such that, for $\nu$-a.e. $s, \mathfrak{S}^{\prime}(s)$ and $\mathfrak{S}^{\prime \prime}(s)$ are subspaces of $\mathfrak{S}_{1}(s) \oplus \mathfrak{S}_{2}(s) \oplus \mathfrak{S}_{3}(s)$ consisting of all $\left(\psi_{1}, \psi_{2}, \psi_{3}\right)$ with $\left(\psi_{1}, \psi_{2}\right) \in G_{\mathcal{R}_{1}(s)}$ and $\left(\psi_{2}, \psi_{3}\right) \in G_{\mathcal{R}_{2}(s)}$ respectively. Clearly, both $\mathfrak{S}^{\prime}$ and $\mathfrak{S}^{\prime \prime}$ are $\nu$-measurable families of closed subspaces of $\mathfrak{S}_{1} \oplus_{\nu} \mathfrak{S}_{2} \oplus_{\nu} \mathfrak{S}_{3}$. Let $\mathcal{S}$ be a $\nu$-a.e. defined map such that, for $\nu$-a.e. $s, \mathcal{S}(s)$ is the everywhere defined bounded operator from $\mathfrak{S}_{1}(s) \oplus \mathfrak{S}_{2}(s) \oplus \mathfrak{S}_{3}(s)$ to $\mathfrak{S}_{1}(s) \oplus \mathfrak{S}_{3}(s)$ taking $\left(\psi_{1}, \psi_{2}, \psi_{3}\right)$ to $\left(\psi_{1}, \psi_{3}\right)$. Then $G_{\mathcal{R}_{2}(s) \mathcal{R}_{1}(s)}$ is the image of $\mathfrak{S}^{\prime}(s) \cap \mathfrak{S}^{\prime \prime}(s)$ under $\mathcal{S}(s)$ for $\nu$-a.e. $s$. Hence, the result follows from Lemma B.4 and statement 2 of Lemma B.6.

Lemma B.9. Let $\mathfrak{S}_{1}$ and $\mathfrak{S}_{2}$ be $\nu$-measurable families of Hilbert spaces and $\mathcal{R}_{1}$ and $\mathcal{R}_{2}$ be $\nu$-measurable families of closed operators from $\mathfrak{S}_{1}$ to $\mathfrak{S}_{2}$. Then $s \rightarrow \mathcal{R}_{2}(s)+\mathcal{R}_{1}(s)$ is a $\nu$-measurable family of operators from $\mathfrak{S}_{1}$ to $\mathfrak{S}_{2}$.

Proof. Let $\mathfrak{S}^{\prime}$ and $\mathfrak{S}^{\prime \prime}$ be $\nu$-a.e. defined maps such that, for $\nu$-a.e. $s, \mathfrak{S}^{\prime}(s)$ and $\mathfrak{S}^{\prime \prime}(s)$ are subspaces of $\mathfrak{S}_{1}(s) \oplus \mathfrak{S}_{2}(s) \oplus \mathfrak{S}_{2}(s)$ consisting of all $\left(\psi_{1}, \psi_{2}, \psi_{3}\right)$ with $\left(\psi_{1}, \psi_{2}\right) \in G_{\mathcal{R}_{1}(s)}$ and $\left(\psi_{1}, \psi_{3}\right) \in G_{\mathcal{R}_{2}(s)}$ respectively. Clearly, both $\mathfrak{S}^{\prime}$ and $\mathfrak{S}^{\prime \prime}$ are $\nu$-measurable families of closed subspaces of $\mathfrak{S}_{1} \oplus_{\nu} \mathfrak{S}_{2} \oplus_{\nu} \mathfrak{S}_{2}$. Let $\mathcal{S}$ be a $\nu$-a.e. defined map such that, for $\nu$-a.e. $s, \mathcal{S}(s)$ is the everywhere defined bounded operator from $\mathfrak{S}_{1}(s) \oplus \mathfrak{S}_{2}(s) \oplus \mathfrak{S}_{2}(s)$ to $\mathfrak{S}_{1}(s) \oplus \mathfrak{S}_{2}(s)$ taking $\left(\psi_{1}, \psi_{2}, \psi_{3}\right)$ to $\left(\psi_{1}, \psi_{2}+\psi_{3}\right)$. Then $G_{\mathcal{R}_{2}(s)+\mathcal{R}_{1}(s)}$ is the image of $\mathfrak{S}^{\prime}(s) \cap \mathfrak{S}^{\prime \prime}(s)$ under $\mathcal{S}(s)$ for $\nu$-a.e. $s$. Hence, the result follows from Lemma B.4 and statement 2 of Lemma B.6.

Lemma B.10. Let $\mathfrak{S}_{1}$ and $\mathfrak{S}_{2}$ be $\nu$-measurable families of Hilbert spaces and $\mathcal{R}$ be a $\nu$-measurable family of closed operators from $\mathfrak{S}_{1}$ to $\mathfrak{S}_{2}$. Let $\xi$ be a $\nu$-measurable section of $\mathfrak{S}_{1}$ such that $\xi(s) \in D_{\mathcal{R}(s)}$ for $\nu$-a.e. $s$. Then $s \rightarrow \mathcal{R}(s) \xi(s)$ is a $\nu$-measurable section of $\mathfrak{S}_{2}$.

Proof. Let $\zeta$ be a $\nu$-a.e. defined section of $\mathfrak{S}_{1} \oplus_{\nu} \mathfrak{S}_{2}$ such that $\zeta(s)=(\xi(s), \mathcal{R}(s) \xi(s))$ for $\nu$-a.e. $s$. It suffices to show that $\zeta$ is $\nu$-measurable. Let $\xi_{1}, \xi_{2}, \ldots$ be a $\nu$-measurable basis in $\mathfrak{S}_{1}$. Let $\mathcal{P}$ be a $\nu$-a.e. defined map such that $\mathcal{P}(s)$ is the orthogonal projection of $\mathfrak{S}_{1}(s) \oplus \mathfrak{S}_{2}(s)$ onto $G_{\mathcal{R}(s)}$ for $\nu$-a.e. $s$. For $j=1,2, \ldots$, let $\zeta_{j}$ be a $\nu$-a.e. defined map such that $\zeta_{j}(s)=\mathcal{P}(s)\left(\xi_{j}(s), 0\right)$ for $\nu$-a.e. $s$. By Lemma B.5. $\mathcal{P}$ is a $\nu$-measurable family of operators in $\mathfrak{S}_{1} \oplus_{\nu} \mathfrak{S}_{2}$, and Lemma B.3 implies that $\zeta_{j}$ is a $\nu$-measurable section of $\mathfrak{S}_{1} \oplus_{\nu} \mathfrak{S}_{2}$ for all $j=1,2, \ldots$. Moreover, the linear span of $\zeta_{1}(s), \zeta_{2}(s), \ldots$ is dense in $G_{\mathcal{R}(s)}$ for $\nu$-a.e. $s \underline{\underline{44}}$ As $\left\langle\zeta_{j}(s), \zeta(s)\right\rangle=\left\langle\xi_{j}(s), \xi(s)\right\rangle$ for $\nu$-a.e. $s$, we conclude that $s \rightarrow\left\langle\zeta_{j}(s), \zeta(s)\right\rangle$ is a $\nu$-measurable function for all $j=1,2, \ldots$. Hence, $\zeta$ is $\nu$-measurable by Lemma B.2.

Remark B.11. The definition of measurability of a family of operators given in this section is mainly the same as in Ref. 8 . The only difference is that, in contrast to Ref. 8 , we do not require operators to be closed and densely defined. This is essential for Propositions B.8 and B.9 because the properties of being closed and densely defined are not inherited by sums and products of operators.

Remark B.12. Let $R$ be a closed operator in a Hilbert space $\mathfrak{H}, P(R)$ be the orthogonal projection of $\mathfrak{H} \oplus \mathfrak{H}$ onto $G_{R}$, and $\pi_{1,2}(\mathfrak{H}): \mathfrak{H} \oplus \mathfrak{H} \rightarrow \mathfrak{H}$ and $j_{1,2}(\mathfrak{H}): \mathfrak{H} \rightarrow \mathfrak{H} \oplus \mathfrak{H}$ be the canonical projections and embeddings respectively. For $i, k=1,2$, we set $P_{i k}(R)=\pi_{i}(\mathfrak{H}) P(R) j_{k}(\mathfrak{H})$. The $2 \times 2$-matrix composed of bounded operators $P_{i k}(R)$ is called the characteristic matrix of (generally, unbounded) operator $R$. In Ref. 7, a $\nu$-a.e. defined family $\mathcal{R}$ of closed operators in a $\nu$-measurable family $\mathfrak{S}$ of Hilbert spaces was called $\nu$-measurable if $s \rightarrow P_{i k}(\mathcal{R}(s)) \xi(s)$ is a $\nu$-measurable section of $\mathfrak{S}$ for all $i, k=1,2$ and every $\nu$-measurable section $\xi$ of $\mathfrak{S}$. It follows from Lemmas B.3 and B.5 that this definition is equivalent to that given in this section because

$$
P(\mathcal{R}(s))=\sum_{i, k=1}^{2} j_{i}(\mathfrak{S}(s)) P_{i k}(\mathcal{R}(s)) \pi_{k}(\mathfrak{S}(s))
$$


for $\nu$-a.e. $s$ and $s \rightarrow \pi_{1,2}(\mathfrak{S}(s))$ and $s \rightarrow j_{1,2}(\mathfrak{S}(s))$ are obviously $\nu$-measurable families (in our sense) of operators from $\mathfrak{S} \oplus_{\nu} \mathfrak{S}$ to $\mathfrak{S}$ and from $\mathfrak{S}$ to $\mathfrak{S} \oplus_{\nu} \mathfrak{S}$ respectively.

Remark B.13. Let $\mathcal{R}$ be a $\nu$-a.e. defined family of operators in a $\nu$-measurable family $\mathfrak{S}$ of Hilbert spaces. Suppose $\mathcal{R}(s)$ is self-adjoint for $\nu$-a.e. $s$. Then $\mathcal{R}(s)+i$ has an everywhere defined bounded inverse for $\nu$-a.e. $s$ and Lemmas B.3 and B.7 imply that $\mathcal{R}$ is $\nu$-measurable if and only if $s \rightarrow(\mathcal{R}(s)+i)^{-1} \xi(s)$ is a $\nu$-measurable section of $\mathfrak{S}$ for any $\nu$-measurable section $\xi$ of $\mathfrak{S}$. In Ref. 45, the last condition was adopted as the definition of measurability for families of self-adjoint operators.

Remark B.14. A $\nu$-a.e. defined family $\mathcal{R}$ of operators in a $\nu$-measurable family $\mathfrak{S}$ of Hilbert spaces is said to be weakly $\nu$-measurable ${ }^{7}$ if $s \rightarrow \mathcal{R}(s) \xi(s)$ is a $\nu$-measurable section of $\mathfrak{S}$ for every $\nu$-measurable section $\xi$ of $\mathfrak{S}$ satisfying $\xi(s) \in D_{\mathcal{R}(s)}$ for $\nu$-a.e. $s$. Lemma B.10 states that every $\nu$-measurable family of closed operators is also weakly $\nu$-measurable. In Ref. 7, where Lemma B.10 was originally proved, a question was posed whether this statement can be reverted, i.e., whether every weakly $\nu$-measurable family of closed operators is $\nu$-measurable. In Ref. 46, it was shown by constructing a counterexample that the answer is negative.

\section{Direct integrals of Hilbert spaces}

Given a $\nu$-measurable family $\mathfrak{S}$ of Hilbert spaces, we denote by $\mathcal{M}(\mathfrak{S}, \nu)$ the set of all $\nu$-equivalence classes $[f]_{\nu}$, where $f$ is a $\nu$-measurable section of $\mathfrak{S}$. Clearly, $\mathcal{M}(\mathfrak{S}, \nu)$ has a natural structure of a complex vector space (for any $\nu$-measurable sections $\xi$ and $\eta$ and any $k \in \mathbb{C}$, we set $[\xi]_{\nu}+[\eta]_{\nu}=[\xi+\eta]_{\nu}$ and $\left.k[\xi]_{\nu}=[k \xi]_{\nu}\right)$. Now suppose $\mathfrak{S}^{\prime}$ is a $\nu$-a.e. defined family of subspaces of $\mathfrak{S}$. We denote by $(\mathfrak{S})-\int^{\oplus} \mathfrak{S}^{\prime}(s) d \nu(s)$ the linear subspace of the space $\mathcal{M}(\mathfrak{S}, \nu)$ consisting of all its elements $\xi$ such that $\xi(s) \in \mathfrak{S}^{\prime}(s)$ for $\nu$-a.e. $s$ and $s \rightarrow\|\xi(s)\|^{2}$ is a $\nu$-integrable function. The space $(\mathfrak{S})-\int^{\oplus} \mathfrak{S}^{\prime}(s) d \nu(s)$ is endowed with the scalar product defined by the relation

$$
\langle\xi, \eta\rangle=\int\langle\xi(s), \eta(s)\rangle d \nu(s)
$$

For any $\nu$-measurable family $\mathfrak{S}$, the space $(\mathfrak{S})-\int^{\oplus} \mathfrak{S}(s) d \nu(s)$ is complete (the proof is essentially the same as that of completeness of ordinary $L_{2}$-spaces) and, hence, is a Hilbert space.

As a rule, the $\nu$-measurable family $\mathfrak{S}$ can be easily deduced from the context. So we usually omit the prefix (S)- and write $\int^{\oplus} \mathfrak{S}^{\prime}(s) d \nu(s)$ in place of $(\mathfrak{S})-\int^{\oplus} \mathfrak{S}^{\prime}(s) d \nu(s)$. The simplest examples of direct integrals are $L_{2}$-spaces and countable direct sums of Hilbert spaces, which can be represented using the constant and discrete families of Hilbert spaces respectively (see Sec. B3). Indeed, for any separable Hilbert space $\mathfrak{h}$, we obviously have

$$
L_{2}\left(S_{\nu}, \mathfrak{h}, \nu\right)=\int^{\oplus} \mathcal{I}_{\mathfrak{h}, \nu}(s) d \nu(s) .
$$

Similarly, if $\mathscr{S}$ is a countable set, $\nu$ is a counting measure on $\mathscr{S}$, and $\mathfrak{S}$ is a $\nu$-a.e. defined family of Hilbert spaces, then

$$
\bigoplus_{s \in \mathscr{S}} \mathfrak{S}(s)=\int^{\oplus} \mathfrak{S}_{\mathscr{S}}(s) d \nu(s)
$$

Given a $\nu$-a.e. defined section $\xi$ of $\mathfrak{S}$ and a $\nu$-measurable set $A$, we denote by $\xi^{A}$ the $\nu$-equivalence class such that $\xi^{A}(s)=\xi(s)$ for $\nu$-a.e. $s \in A$ and $\xi^{A}(s)=0$ for $\nu$-a.e. $s \in S_{\nu} \backslash A$.

Lemma B.15. Let $\mathfrak{S}$ be a $\nu$-measurable family of Hilbert spaces and $\mathfrak{S}^{\prime}$ be a $\nu$-measurable family of subspaces of $\mathfrak{S}$. Then there is a $\nu$-measurable basis in $\mathfrak{S}^{\prime}$ consisting of elements of $\int^{\oplus} \mathfrak{S}^{\prime}(s) d \nu(s)$.

Proof. Let $\xi_{1}, \xi_{2}, \ldots$ be a $\nu$-measurable basis in $\mathfrak{S}^{\prime}$. Multiplying $\xi_{j}$ by suitable $\nu$-measurable functions, we can ensure that $\left\|\xi_{j}(s)\right\| \leq 1$ for all $j=1,2, \ldots$ and $\nu$-a.e. $s$. Let $A_{1}, A_{2}, \ldots$ be elements of $D_{\nu}$ such that $S_{\nu}=\bigcup_{k=1}^{\infty} A_{k}$. Then $\xi_{j}^{A_{k}}$ with $j, k=1,2, \ldots$ obviously constitute the required $\nu$-measurable basis in $\mathfrak{S}^{\prime}$.

Lemma B.16. Let $\mathfrak{S}$ and $\mathfrak{S}^{\prime}$ be as in Lemma B.15 and $\mathfrak{H}^{\prime}=\int \mathfrak{S}^{\prime}(s) d \nu(s)$. Then $\mathfrak{H}^{\prime \perp}=$ $\int^{\oplus} \mathfrak{S}^{\prime}(s)^{\perp} d \nu(s)$ and $\overline{\mathfrak{H}^{\prime}}=\iint^{\oplus} \overline{\mathfrak{S}^{\prime}(s)} d \nu(s)$. 
Proof. Let $\eta \in \mathfrak{H}^{\prime \perp}$ and $\xi \in \mathfrak{H}^{\prime}$. As $\xi^{A} \in \mathfrak{H}^{\prime}$ for any $A \in D_{\nu}$, we obtain $\int_{A}\langle\xi(s), \eta(s)\rangle d \nu(s)=\left\langle\xi_{A}, \eta\right\rangle=0$ and, hence, $\langle\xi(s), \eta(s)\rangle=0$ for $\nu$-a.e. $s$. By Lemma B.15 there is a $\nu$-measurable basis $\xi_{1}, \xi_{2}, \ldots$ in $\mathfrak{S}^{\prime}$ such that $\xi_{j} \in \mathfrak{H}^{\prime}$ for all $j=1,2, \ldots$. Since $\left\langle\xi_{j}(s), \eta(s)\right\rangle=0$ for $\nu$-a.e. $s$ and all $j=1,2, \ldots$, we have $\eta(s) \in \mathfrak{S}^{\prime}(s)^{\perp}$ for $\nu$-a.e. $s$. This implies that $\mathfrak{H}^{\prime \perp}=\int^{\oplus} \mathfrak{S}^{\prime}(s)^{\perp} d \nu(s)$. The equality for the closure of $\mathfrak{H}^{\prime}$ now follows from the relations $\overline{\mathfrak{H}^{\prime}}=\left(\mathfrak{H}^{\perp}\right)^{\perp}$ and $\overline{\mathfrak{S}^{\prime}(s)}=\left(\mathfrak{S}^{\prime}(s)^{\perp}\right)^{\perp}$.

Let $\mathfrak{S}$ be a $\nu$-measurable family of Hilbert spaces and $\mathfrak{H}=\int{ }^{\oplus} \mathfrak{S}(s) d \nu(s)$. Given a $\nu$-measurable complex function $g$, we denote by $\mathcal{T}_{g}^{\nu, \mathfrak{S}}$ the operator of multiplication by $g$ in $\mathfrak{H}$. By definition, the graph of $\mathcal{T}_{g}^{\nu, \mathfrak{S}}$ consists of all pairs $\left(\xi_{1}, \xi_{2}\right) \in \mathfrak{H} \oplus \mathfrak{H}$ such that $\xi_{2}(s)=g(s) \xi_{1}(s)$ for $\nu$-a.e. $s$. The operator $\mathcal{T}_{g}^{\nu, \mathfrak{S}}$ is closed and densely defined and its adjoint is equal to $\mathcal{T}_{\bar{g}}^{\nu, \mathfrak{S}}$, where $\bar{g}$ is the complex conjugate function of $g$. In particular, if $g$ is real, then $\mathcal{T}_{g}^{\nu, \mathfrak{S}}$ is self-adjoint. If $g$ is $\nu$-essentially bounded, then $\mathcal{T}_{g}^{\nu, \mathfrak{S}}$ is everywhere defined and bounded.

Lemma B.17. Let $\mathfrak{S}$ be a $\nu$-measurable family of Hilbert spaces, $\mathfrak{H}=\int{ }^{\oplus} \mathfrak{S}(s) d \nu(s)$, and $\mathfrak{H}^{\prime}$ be a closed linear subspace of $\mathfrak{H}$ such that $\mathcal{T}_{g}^{\nu, \mathfrak{S}} \xi \in \mathfrak{H}^{\prime}$ for any $\xi \in \mathfrak{H}^{\prime}$ and every $\nu$-essentially bounded complex function g. Then there is a $\nu$-measurable family $\mathfrak{S}^{\prime}$ of closed subspaces of $\mathfrak{S}$ such that $\mathfrak{H}^{\prime}=\int^{\oplus} \mathfrak{S}^{\prime}(s) d \nu(s)$.

Proof. By the hypothesis, the projection $P$ of $\mathfrak{H}$ onto $\mathfrak{H}^{\prime}$ commutes with $\mathcal{T}_{g}^{\nu, \mathfrak{S}}$ for every $\nu$-essentially bounded function $g$. By Lemma B.15, there is a $\nu$-measurable basis $\xi_{1}, \xi_{2}, \ldots$ in $\mathfrak{S}$ consisting of elements of $\mathfrak{H}$. For each $j=1,2, \ldots$, we set $\eta_{j}=P \xi_{j}$. Let $\mathfrak{S}^{\prime}$ be a $\nu$-measurable family of subspaces of $\mathfrak{S}$ such that $\mathfrak{S}^{\prime}(s)$ is the closed linear span of $\eta_{1}(s), \eta_{2}(s), \ldots$ for $\nu$-a.e. $s$. Let $\tilde{\mathfrak{H}}=\int^{\oplus} \mathfrak{S}^{\prime}(s) d \nu(s)$. If $\xi \in \mathfrak{H}$ is such that $\left\langle\xi_{j}^{A}, \xi\right\rangle=\int_{A}\left\langle\xi_{j}(s), \xi(s)\right\rangle d \nu(s)=0$ for every $\nu$-measurable set $A$ and $j=1,2, \ldots$, then $\left\langle\xi_{j}(s), \xi(s)\right\rangle=0$ for all $j$ and $\nu$-a.e. $s$ and, therefore, $\xi=0$. This means that the linear span of all $\xi_{j}^{A}$ is dense in $\mathfrak{H}$. Note that $\eta_{j}^{A}=\mathcal{T}_{\chi A}^{\nu, S} P \xi_{j}=P \xi_{j}^{A}$, where $\chi_{A}(s)=1$ for $s \in A$ and $\chi_{A}(s)=0$ for $s \in S_{\nu} \backslash A$. Hence, the linear span of $\eta_{j}^{A}$ is dense in $\mathfrak{H}^{\prime}$. Since $\tilde{\mathfrak{H}}$ is closed and contains all $\eta_{j}^{A}$, we conclude that $\mathfrak{H}^{\prime} \subset \tilde{\mathfrak{H}}$. Let $\eta \in \mathfrak{H}^{\prime \perp}$. Then we have $\left\langle\eta_{j}^{A}, \eta\right\rangle=\int_{A}\left\langle\eta_{j}(s), \eta(s)\right\rangle d \nu(s)=0$ and, hence, $\left\langle\eta_{j}(s), \eta(s)\right\rangle=0$ for all $j$ and $\nu$-a.e. $s$. This implies that $\eta(s) \in \mathfrak{S}^{\prime}(s)^{\perp}$ for $\nu$-a.e. $s$, i.e., $\eta \in \tilde{\mathfrak{H}}^{\perp}$. We therefore obtain $\mathfrak{H}^{\perp} \subset \tilde{\mathfrak{H}}^{\perp}$. As $\mathfrak{H}^{\prime}$ is closed, it follows that $\tilde{\mathfrak{H}} \subset \mathfrak{H}^{\prime}$ and, hence, $\tilde{\mathfrak{H}}=\mathfrak{H}^{\prime}$.

\section{Direct integrals of operators}

Let $\mathfrak{S}_{1}$ and $\mathfrak{S}_{2}$ be $\nu$-measurable families of Hilbert spaces. Let $\mathfrak{H}_{1,2}=\int \mathfrak{S}_{1,2}(s) d \nu(s)$ and $\mathcal{R}$ be a $\nu$-a.e. defined family of operators from $\mathfrak{S}_{1}$ to $\mathfrak{S}_{2}$. The direct integral $\int^{\oplus} \mathcal{R}(s) d \nu(s)$ of the family $\mathcal{R}$ is defined as the linear operator from $\mathfrak{H}_{1}$ to $\mathfrak{H}_{2}$ whose graph consists of all pairs $(\xi, \eta) \in \mathfrak{H}_{1} \oplus \mathfrak{H}_{2}$ such that $\xi(s) \in D_{\mathcal{R}(s)}$ and $\mathcal{R}(s) \xi(s)=\eta(s)$ for $\nu$-a.e. $s$.

Let $\mathfrak{H}=\int^{\oplus} \mathfrak{S}_{1}(s) \oplus \mathfrak{S}_{2}(s) d \nu(s)$. Then there is a unique unitary operator $U_{\mathfrak{S}_{1}, \mathfrak{S}_{2}}: \mathfrak{H}_{1} \oplus \mathfrak{H}_{2} \rightarrow \mathfrak{H}$ such that $\left(U_{\mathfrak{S}_{1}, \mathfrak{S}_{2}}(\xi, \eta)\right)(s)=(\xi(s), \eta(s))$ for any $(\xi, \eta) \in \mathfrak{H}_{1} \oplus \mathfrak{H}_{2}$ and $\nu$-a.e. $s$. We call $U_{\mathfrak{S}_{1}, \mathfrak{S}_{2}}$ the natural isomorphism between $\mathfrak{H}_{1} \oplus \mathfrak{H}_{2}$ and $\mathfrak{H}$.

The next statement follows immediately from the definition of $U_{\mathfrak{S}_{1}, \mathfrak{S}_{2}}$.

Lemma B.18. Let $\mathfrak{S}_{1}$ and $\mathfrak{S}_{2}$ be $\nu$-measurable families of Hilbert spaces, $\mathcal{R}$ be a $\nu$-a.e. defined family of operators from $\mathfrak{S}_{1}$ to $\mathfrak{S}_{2}$, and $R=\int^{\oplus} \mathcal{R}(s) d \nu(s)$. Then we have $U_{\mathfrak{S}_{1}, \mathfrak{S}_{2}}\left(G_{R}\right)=\left(\mathfrak{S}_{1} \oplus_{\nu}\right.$ $\left.\mathfrak{S}_{2}\right)-\int^{\oplus} G_{\mathcal{R}(s)} d \nu(s)$.

Lemma B.19. Let $\mathfrak{H}_{1,2}=\int{ }^{\oplus} \mathfrak{S}_{1,2}(s) d \nu(s)$, where $\mathfrak{S}_{1}$ and $\mathfrak{S}_{2}$ are $\nu$-measurable families of Hilbert spaces, and $G$ be a closed linear subspace of $\mathfrak{H}_{1} \oplus \mathfrak{H}_{2}$. Suppose $\mathcal{G}$ is a $\nu$-measurable family of closed subspaces of $\mathfrak{S}_{1} \oplus_{\nu} \mathfrak{S}_{2}$ such that $U_{\mathfrak{S}_{1}, \mathfrak{S}_{2}}(G)=\int^{\oplus} \mathcal{G}(s) d \nu(s)$. Then $G$ is a graph of an operator from $\mathfrak{H}_{1}$ to $\mathfrak{H}_{2}$ if and only if $\mathcal{G}(s)$ is a graph of an operator from $\mathfrak{S}_{1}(s)$ to $\mathfrak{S}_{2}(s)$ for $\nu$-a.e. $s$.

Proof. Let $U=U_{\mathfrak{S}_{1}, \mathfrak{S}_{2}}$ and $\mathfrak{S}^{\prime}$ be a $\nu$-a.e. defined map such that $\mathfrak{S}^{\prime}(s)=\{0\} \times \mathfrak{S}_{2}(s)$ for $\nu$-a.e. $s$. Clearly, $\mathfrak{S}^{\prime}$ is a $\nu$-measurable family of closed subspaces of $\mathfrak{S}_{1} \oplus_{\nu} \mathfrak{S}_{2}$. Let $\mathfrak{H}^{\prime}=\int^{\oplus} \mathfrak{S}^{\prime}(s) d \nu(s)$ and $\mathfrak{H}^{\prime \prime}=\int \mathfrak{S}^{\prime}(s) \cap \mathcal{G}(s) d \nu(s)$. Since $\mathfrak{H}^{\prime \prime}=\mathfrak{H}^{\prime} \cap U(G)$ and $U^{-1}\left(\mathfrak{H}^{\prime}\right)=\{0\} \times \mathfrak{H}_{2}$, we have $G \cap\left(\{0\} \times \mathfrak{H}_{2}\right)=$ $U^{-1}\left(\mathfrak{H}^{\prime \prime}\right)$. Hence, $G$ is a graph of an operator if and only if $\mathfrak{H}^{\prime \prime}$ is trivial. By statement 2 of Lemma B.6, $s \rightarrow \mathfrak{S}^{\prime}(s) \cap \mathcal{G}(s)$ is a $\nu$-measurable family of subspaces of $\mathfrak{S}_{1} \oplus_{\nu} \mathfrak{S}_{2}$ and, therefore, $\mathfrak{H}^{\prime \prime}$ is trivial if and only if $\mathfrak{S}^{\prime}(s) \cap \mathcal{G}(s)$ is trivial for $\nu$-a.e. $s$, i.e., if $\mathcal{G}(s)$ is a graph of an operator for $\nu$-a.e. $s$. 
Lemma B.20. Let $\mathfrak{S}_{1}$ and $\mathfrak{S}_{2}$ be $\nu$-measurable families of Hilbert spaces, $\mathcal{R}$ be a $\nu$-measurable family of operators from $\mathfrak{S}_{1}$ to $\mathfrak{S}_{2}$, and $R=\int^{\oplus} \mathcal{R}(s) d \nu(s)$. Then $R$ is closable if and only if $\mathcal{R}(s)$ is closable for $\nu$-a.e. $s$, in which case $\bar{R}=\int^{\oplus} \overline{\mathcal{R}(s)} d \nu(s)$.

Proof. Let $U=U_{\mathfrak{S}_{1}, \mathfrak{S}_{2}}$. As $U\left(G_{R}\right)=\int^{\oplus} G_{\mathcal{R}(s)} d \nu(s)$, Lemma B.16 yields

$$
U\left(\bar{G}_{R}\right)=\int^{\oplus} \bar{G}_{\mathcal{R}(s)} d \nu(s) .
$$

The operator $R$ is closable if and only if $\bar{G}_{R}$ is a graph of an operator. By Lemma B.19, the latter condition holds if and only if $\bar{G}_{\mathcal{R}(s)}$ is a graph of an operator for $\nu$-a.e. $s$, i.e., if $\mathcal{R}(s)$ is closable for $\nu$-a.e. $s$. Suppose now that $R$ is closable and $R^{\prime}=\int \oplus \overline{\mathcal{R}(s)} d \nu(s)$. As $\bar{G}_{R}=G_{\bar{R}}$ and $\bar{G}_{\mathcal{R}(s)}=G_{\overline{\mathcal{R}(s)}}$ for $\nu$-a.e. $s$, equality (B3) and Lemma B.18 imply that $U\left(G_{\bar{R}}\right)=U\left(G_{R^{\prime}}\right)$ and, hence, $R=R^{\prime}$.

Proposition B.21. Let $\mathfrak{S}_{1}$ and $\mathfrak{S}_{2}$ be $\nu$-measurable families of Hilbert spaces and $\mathcal{R}$ and $\mathcal{R}^{\prime}$ be $\nu$ measurable families of closed operators from $\mathfrak{S}_{1}$ to $\mathfrak{S}_{2}$. Let $R=\int^{\oplus} \mathcal{R}(s) d \nu(s)$, and $R^{\prime}=\int^{\oplus} \mathcal{R}^{\prime}(s) d \nu(s)$. If $R$ is an extension of $R^{\prime}$, then $\mathcal{R}(s)$ is an extension of $\mathcal{R}^{\prime}(s)$ for $\nu$-a.e. s. If $R=R^{\prime}$, then $\mathcal{R}(s)=\mathcal{R}^{\prime}(s)$ for $\nu$-a.e. $s$.

Proof. Let $U=U_{\mathfrak{S}_{1}, \mathfrak{S}_{2}}$. Since $U\left(G_{R^{\prime}}\right)=\int{ }^{\oplus} G_{\mathcal{R}^{\prime}(s)} d \nu(s)$, Lemma B.15 implies that there exists a sequence $\zeta_{1}, \zeta_{2}, \ldots$ of elements of $U\left(G_{R^{\prime}}\right)$ such that the linear span of $\zeta_{1}(s), \zeta_{2}(s), \ldots$ is dense in $G_{\mathcal{R}^{\prime}(s)}$ for $\nu$-a.e. $s$. If $R$ is an extension of $R^{\prime}$, then $U\left(G_{R^{\prime}}\right) \subset U\left(G_{R}\right)$ and, hence, $\zeta_{j} \in U\left(G_{R}\right)$ for all $j=1,2, \ldots$ Since $U\left(G_{R}\right)=\int^{\oplus} G_{\mathcal{R}(s)} d \nu(s)$, it follows that $\zeta_{j}(s) \in G_{\mathcal{R}(s)}$ for $\nu$-a.e. $s$ and all $j=1,2, \ldots$ The linear span of $\zeta_{1}(s), \zeta_{2}(s), \ldots$ is therefore contained in $G_{\mathcal{R}(s)}$ for $\nu$-a.e. $s$. As $\mathcal{R}(s)$ is closed, it follows that $G_{\mathcal{R}^{\prime}(s)} \subset G_{\mathcal{R}(s)}$, i.e., $\mathcal{R}(s)$ is an extension of $\mathcal{R}^{\prime}(s)$ for $\nu$-a.e. $s$. If $R=R^{\prime}$, then, by the above, $\mathcal{R}(s)$ and $\mathcal{R}^{\prime}(s)$ are extensions of each other and, therefore, are equal for $\nu$-a.e. $s$.

Proposition B.22. Let $\mathfrak{S}_{1}, \mathfrak{S}_{2}, \mathfrak{H}_{1}$, and $\mathfrak{H}_{2}$ be as in Lemma B.19. Let $\mathcal{R}$ be a $\nu$-measurable family of closed operators from $\mathfrak{S}_{1}$ to $\mathfrak{S}_{2}$. Then $R=\int^{\oplus} \mathcal{R}(s) d \nu(s)$ is a closed operator from $\mathfrak{H}_{1}$ to $\mathfrak{H}_{2}$ satisfying the condition:

(M) If $\xi \in D_{R}$ and $g$ is a $\nu$-measurable $\nu$-essentially bounded complex function, then $\mathcal{T}_{g}^{\nu, \mathfrak{S}_{1}} \xi \in D_{R}$ and $R \mathcal{T}_{g}^{\nu, \mathfrak{S}_{1}} \xi=\mathcal{T}_{g}^{\nu, \mathfrak{S}_{2}} R \xi$

Conversely, if $R$ is a closed operator from $\mathfrak{H}_{1}$ to $\mathfrak{H}_{2}$ satisfying $(\mathrm{M})$, then there is a unique (up to $\nu$ equivalence) $\nu$-measurable family $\mathcal{R}$ of closed operators from $\mathfrak{S}_{1}$ to $\mathfrak{S}_{2}$ such that $R=\int^{\oplus} \mathcal{R}(s) d \nu(s)$.

Proof. Let $U=U_{\mathfrak{S}_{1}, \mathfrak{S}_{2}}, \mathcal{R}$ be a $\nu$-measurable family of closed operators from $\mathfrak{S}_{1}$ to $\mathfrak{S}_{2}$, and $R=$ $\int^{\oplus} \mathcal{R}(s) d \nu(s)$. By Lemma B.20, $R$ is closed. If $\xi \in D_{R}$ and $g$ is a $\nu$-measurable $\nu$-essentially bounded function, then $\left(\mathcal{T}_{g}^{\nu, \mathfrak{S}_{1}} \xi, \mathcal{T}_{g}^{\nu, \mathfrak{S}_{2}} R \xi\right)$ belongs to $G_{R}$ by the very definition of the direct integral of operators. This means that $(\mathrm{M})$ is fulfilled. Conversely, let $R$ be a closed operator from $\mathfrak{H}_{1}$ to $\mathfrak{H}_{2}$ satisfying (M). If $(\xi, \eta) \in G_{R}$ and $g$ is a $\nu$-measurable $\nu$-essentially bounded function, then $\left(\mathcal{T}_{g}^{\nu, \mathfrak{S}_{1}} \xi, \mathcal{T}_{g}^{\nu, \mathfrak{S}_{2}} \eta\right)$ belongs to $G_{R}$ by $(\mathrm{M})$ and, hence, $\mathcal{T}_{g}^{\nu, \mathfrak{S}_{1} \oplus_{\nu} \mathfrak{S}_{2}} U(\xi, \eta)=U\left(\mathcal{T}_{g}^{\nu, \mathfrak{S}_{1}} \xi, \mathcal{T}_{g}^{\nu, \mathfrak{S}_{2}} \eta\right)$ belongs to $U\left(G_{R}\right)$. This means that $U\left(G_{R}\right)$

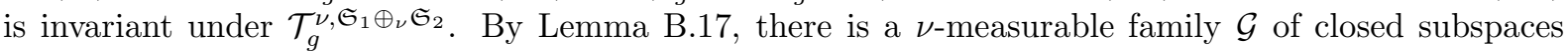
of $\mathfrak{S}_{1} \oplus_{\nu} \mathfrak{S}_{2}$ such that $U\left(G_{R}\right)=\int^{\oplus} \mathcal{G}(s) d \nu(s)$. By Lemma B.19 there is a $\nu$-measurable family $\mathcal{R}$ of closed operators from $\mathfrak{S}_{1}$ to $\mathfrak{S}_{2}$ such that $\mathcal{G}(s)=G_{\mathcal{R}(s)}$ for $\nu$-a.e. $s$. If $R^{\prime}=\int^{\oplus} \mathcal{R}(s) d \nu(s)$, then it follows from Lemma B.18 that $U\left(G_{R^{\prime}}\right)=U\left(G_{R}\right)$ and, hence, $R=R^{\prime}$. The uniqueness of $\mathcal{R}$ is ensured by Proposition B.21.

Let $\mathfrak{H}_{1}$ and $\mathfrak{H}_{2}$ be Hilbert spaces and $R$ be an operator from $\mathfrak{H}_{1}$ to $\mathfrak{H}_{2}$. We define the linear subspaces $G_{R}^{\circ}$ and $G_{R}^{*}$ of $\mathfrak{H}_{2} \oplus \mathfrak{H}_{1}$ by setting

$$
G_{R}^{\circ}=\left\{\left(-\psi_{2}, \psi_{1}\right):\left(\psi_{1}, \psi_{2}\right) \in G_{R}\right\}, \quad G_{R}^{*}=\left(G_{R}^{\circ}\right)^{\perp} .
$$

The operator $R$ is densely defined if and only if $G_{R}^{*}$ is the graph of an operator, in which case $G_{R^{*}}=G_{R}^{*}$.

Proposition B.23. Let $\mathfrak{S}_{1}$ and $\mathfrak{S}_{2}$ be $\nu$-measurable families of Hilbert spaces, $\mathcal{R}$ be a $\nu$-measurable family of operators from $\mathfrak{S}_{1}$ to $\mathfrak{S}_{2}$, and $R=\int^{\oplus} \mathcal{R}(s) d \nu(s)$. Then the following statements hold:

1. Suppose $\mathcal{R}(s)$ is closed for $\nu$-a.e. s. The operator $R$ is invertible if and only if $\mathcal{R}(s)$ is invertible for $\nu$-a.e. $s$, in which case $s \rightarrow \mathcal{R}(s)^{-1}$ is a $\nu$-measurable family of operators from $\mathfrak{S}_{2}$ to $\mathfrak{S}_{1}$ and $R^{-1}=\int^{\oplus} \mathcal{R}(s)^{-1} d \nu(s)$. 
2. The operator $R$ is densely defined if and only if $\mathcal{R}(s)$ is densely defined for $\nu$-a.e. $s$, in which case $s \rightarrow \mathcal{R}(s)^{*}$ is a $\nu$-measurable family of operators from $\mathfrak{S}_{2}$ to $\mathfrak{S}_{1}$ and $R^{*}=\int{ }^{\oplus} \mathcal{R}(s)^{*} d \nu(s)$.

Proof. Let $\mathfrak{H}_{1,2}=\int^{\oplus} \mathfrak{S}_{1,2}(s) d \nu(s)$.

1. By statement 2 of Lemma B.7, $s \rightarrow \operatorname{Ker} \mathcal{R}(s)$ is a $\nu$-measurable family of subspaces of $\mathfrak{S}_{1}$. As $\operatorname{Ker} R=\int{ }^{\oplus} \operatorname{Ker} \mathcal{R}(s) d \nu(s)$, we conclude that $R$ is invertible if and only if $\operatorname{Ker} \mathcal{R}(s)=\{0\}$ for $\nu$-a.e. $s$, i.e., if $\mathcal{R}(s)$ is invertible for $\nu$-a.e. $s$. Let $R$ be invertible and $R^{\prime}=\int{ }^{\oplus} \mathcal{R}(s)^{-1} d \nu(s)$. For any $(\eta, \xi) \in \mathfrak{H}_{2} \oplus \mathfrak{H}_{1}$, we have

$$
\begin{aligned}
(\eta, \xi) \in G_{R^{-1}} \Longleftrightarrow(\xi, \eta) \in G_{R} \Longleftrightarrow(\xi(s), \eta(s)) \in G_{\mathcal{R}(s)} \text { for } \nu \text {-a.e. } s \Longleftrightarrow & \\
& (\eta(s), \xi(s)) \in G_{\mathcal{R}(s)^{-1}} \text { for } \nu \text {-a.e. } s \Longleftrightarrow(\eta, \xi) \in G_{R^{\prime}} .
\end{aligned}
$$

We thus have $G_{R^{-1}}=G_{R^{\prime}}$ and, hence, $R=R^{\prime}$. By statement 1 of Lemma B.7 $s \rightarrow \mathcal{R}(s)^{-1}$ is a $\nu$-measurable family of operators from $\mathfrak{S}_{2}$ to $\mathfrak{S}_{1}$.

2. Let $U_{12}=U_{\mathfrak{S}_{1}, \mathfrak{S}_{2}}$ and $U_{21}=U_{\mathfrak{S}_{2}, \mathfrak{S}_{1}}$. For $\nu$-a.e. $s$, let $\mathcal{S}(s)$ be everywhere defined bounded operator from $\mathfrak{S}_{1}(s) \oplus \mathfrak{S}_{2}(s)$ to $\mathfrak{S}_{2}(s) \oplus \mathfrak{S}_{1}(s)$ taking $\left(\psi_{1}, \psi_{2}\right)$ to $\left(-\psi_{2}, \psi_{1}\right)$. Clearly, $\mathcal{S}$ is a $\nu$-measurable family of unitary operators from $\mathfrak{S}_{1} \oplus_{\nu} \mathfrak{S}_{2}$ to $\mathfrak{S}_{2} \oplus_{\nu} \mathfrak{S}_{1}$. Note that $G_{\mathcal{R}(s)}^{\circ}$ is the image of $G_{\mathcal{R}(s)}$ under $\mathcal{S}(s)$ for $\nu$-a.e. $s$. Hence, $s \rightarrow G_{\mathcal{R}(s)}^{\circ}$ is a $\nu$-measurable family of subspaces of $\mathfrak{S}_{2} \oplus_{\nu} \mathfrak{S}_{1}$ by Lemma B.4. Let $S=\int{ }^{\oplus} \mathcal{S}(s) d \nu(s)$ and $\tilde{S}$ be the unitary operator from $\mathfrak{H}_{1} \oplus \mathfrak{H}_{2}$ to $\mathfrak{H}_{2} \oplus \mathfrak{H}_{1}$ defined by the relation $\tilde{S}(\xi, \eta)=(-\eta, \xi)$. It is straightforward to check that $U_{21} \tilde{S}=S U_{12}$. As $G_{R}^{\circ}=\tilde{S}\left(G_{R}\right)$, we have $U_{21}\left(G_{R}^{\circ}\right)=$ $S\left(U_{12}\left(G_{R}\right)\right)$. Since $U_{12}\left(G_{R}\right)=\int{ }^{\oplus} G_{\mathcal{R}(s)} d \nu(s)$ by Lemma B.18 and the image of $\int^{\oplus} G_{\mathcal{R}(s)} d \nu(s)$ under $S$ is equal to $\int{ }^{\oplus} G_{\mathcal{R}(s)}^{\circ} d \nu(s)$, we conclude that $U_{21}\left(G_{R}^{\circ}\right)=\int{ }^{\oplus} G_{\mathcal{R}(s)}^{\circ} d \nu(s)$. It now follows from Lemmas B.6 and B.16 and the unitarity of $U_{21}$ that $s \rightarrow G_{\mathcal{R}(s)}^{*}$ is a $\nu$-measurable family of subspaces of $\mathfrak{S}_{2} \oplus_{\nu} \mathfrak{S}_{1}$ and

$$
U_{21}\left(G_{R}^{*}\right)=\int^{\oplus} G_{\mathcal{R}(s)}^{*} d \nu(s)
$$

In view of (B4), Lemma B.19 implies that $R$ is densely defined if and only if $G_{\mathcal{R}(s)}^{*}$ is the graph of an operator for $\nu$-a.e. $s$, i.e., if $\mathcal{R}(s)$ is densely defined for $\nu$-a.e. $s$. Suppose $R$ is densely defined and $R^{\prime}=\int^{\oplus} \mathcal{R}(s)^{*} d \nu(s)$. As $G_{\mathcal{R}(s)}^{*}=G_{\mathcal{R}(s)^{*}}$ for $\nu$-a.e. $s$, it follows that $s \rightarrow \mathcal{R}(s)^{*}$ is a $\nu$-measurable family of operators from $\mathfrak{S}_{2}$ to $\mathfrak{S}_{1}$. Finally, since $G_{R}^{*}=G_{R^{*}}$, equality (B4) and Lemma B.18 imply that $U_{21}\left(G_{R^{*}}\right)=U_{21}\left(G_{R^{\prime}}\right)$ and, hence, $R^{*}=R^{\prime}$.

Corollary B.24. Let $\mathfrak{S}$ be a $\nu$-measurable family of Hilbert spaces, and $\mathcal{R}$ be a $\nu$-measurable family of closed operators in $\mathfrak{S}$. The operator $\int^{\oplus} \mathcal{R}(s) d \nu(s)$ is self-adjoint if and only if $\mathcal{R}(s)$ is self-adjoint for $\nu$-a.e. s.

Proof. The statement follows from Propositions B.23 and B.21.

Proposition B.25. Let $\mathfrak{S}_{1}, \mathfrak{S}_{2}$, and $\mathfrak{S}_{3}$ be $\nu$-measurable families of Hilbert spaces and $\mathcal{R}_{1}$ and $\mathcal{R}_{2}$ be $\nu$-measurable families of closed operators from $\mathfrak{S}_{1}$ to $\mathfrak{S}_{2}$ and from $\mathfrak{S}_{2}$ to $\mathfrak{S}_{3}$ respectively. Let $R_{1,2}=$ $\int^{\oplus} \mathcal{R}_{1,2}(s) d \nu(s)$. The operator $R_{2} R_{1}$ is densely defined if and only if the operator $\mathcal{R}_{2}(s) \mathcal{R}_{1}(s)$ is densely defined for $\nu$-a.e. $s$. The operator $R_{2} R_{1}$ is closable if and only if the operator $\mathcal{R}_{2}(s) \mathcal{R}_{1}(s)$ is closable for $\nu$-a.e. $s$, in which case $s \rightarrow \overline{\mathcal{R}_{2}(s) \mathcal{R}_{1}(s)}$ is a $\nu$-measurable family of operators from $\mathfrak{S}_{1}$ to $\mathfrak{S}_{3}$ and $\overline{R_{2} R_{1}}=\int{ }^{\oplus} \overline{\mathcal{R}_{2}(s) \mathcal{R}_{1}(s)} d \nu(s)$.

Proof. Let $\mathfrak{H}_{i}=\int^{\oplus} \mathfrak{S}_{i}(s) d \nu(s), i=1,2,3$, and $R=\int{ }^{\oplus} \mathcal{R}_{2}(s) \mathcal{R}_{1}(s) d \nu(s)$. Clearly, $R$ is an extension of $R_{2} R_{1}$. Let $(\xi, \eta) \in G_{R}$. Then $\xi(s) \in D_{\mathcal{R}_{2}(s) \mathcal{R}_{1}(s)}$ and, hence, $\xi(s) \in D_{\mathcal{R}_{1}(s)}$ for $\nu$-a.e. $s$. Let $\tilde{\eta}$ be a $\nu$-a.e. defined section of $\mathfrak{S}_{2}$ such that $\tilde{\eta}(s)=\mathcal{R}_{1}(s) \xi(s)$ for $\nu$-a.e. $s$. By Lemma B.10 $\tilde{\eta}$ is $\nu$-measurable. Let $A_{1} \subset A_{2} \subset \ldots$ be a sequence of elements of $D_{\nu}$ such that $S_{\nu}=\bigcup_{k=1}^{\infty} A_{k}$ and $\|\tilde{\eta}(s)\| \leq k$ for $\nu$-a.e. $s \in A_{k}$. Set $\xi_{k}=\xi^{A_{k}}, \eta_{k}=\eta^{A_{k}}$, and $\tilde{\eta}_{k}=\tilde{\eta}^{A_{k}}$ We obviously have $\xi_{k} \in \mathfrak{H}_{1}, \tilde{\eta}_{k} \in \mathfrak{H}_{2}$, and $\eta_{k} \in \mathfrak{H}_{3}$ for all $k$. As $\tilde{\eta}(s) \in D_{\mathcal{R}_{2}(s)}$ and $\eta(s)=\mathcal{R}_{2}(s) \tilde{\eta}(s)$ for $\nu$-a.e. $s$, we have $\left(\xi_{k}, \tilde{\eta}_{k}\right) \in G_{R_{1}}$ and $\left(\tilde{\eta}_{k}, \eta_{k}\right) \in G_{R_{2}}$ and, therefore, $\left(\xi_{k}, \eta_{k}\right) \in G_{R_{2} R_{1}}$ for all $k$. Since $\left(\xi_{k}, \eta_{k}\right) \rightarrow(\xi, \eta)$ in $\mathfrak{H}_{1} \oplus \mathfrak{H}_{3}$ as $k \rightarrow \infty$, we conclude that $G_{R_{2} R_{1}}$ is dense in $G_{R}$ and, hence, $D_{R_{2} R_{1}}$ is dense in $D_{R}$. In view of statement 2 of Proposition B.23, it follows that $R_{2} R_{1}$ is densely defined if and only if $\mathcal{R}_{2}(s) \mathcal{R}_{1}(s)$ is densely defined for $\nu$-a.e. $s$. Since $\bar{G}_{R}=\bar{G}_{R_{2} R_{1}}$, the operator $R_{2} R_{1}$ is closable if and only if $R$ is closable. In view of Lemma B.20, the latter condition holds if and only if $\mathcal{R}_{2}(s) \mathcal{R}_{1}(s)$ is closable for $\nu$-a.e. $s$ (note that $s \rightarrow R_{2}(s) \mathcal{R}_{1}(s)$ is a $\nu$-measurable family of operators from $\mathfrak{S}_{1}$ to $\mathfrak{S}_{3}$ by Lemma B.8). If $R_{2} R_{1}$ is closable, then Lemma B.20 implies that $\overline{R_{2} R_{1}}=\bar{R}=\int{ }^{\oplus} \overline{\mathcal{R}_{2}(s) \mathcal{R}_{1}(s)} d \nu(s)$. 
Proposition B.26. Let $\mathfrak{S}_{1}$ and $\mathfrak{S}_{2}$ be $\nu$-measurable families of Hilbert spaces, $\mathcal{R}_{1}$ and $\mathcal{R}_{2}$ be $\nu$-measurable families of closed operators from $\mathfrak{S}_{1}$ to $\mathfrak{S}_{2}$, and $R_{1,2}=\int^{\oplus} \mathcal{R}_{1,2}(s) d \nu(s)$. The operator $R_{1}+R_{2}$ is densely defined if and only if the operator $\mathcal{R}_{1}(s)+\mathcal{R}_{2}(s)$ is densely defined for $\nu$-a.e. s. The operator $R_{1}+R_{2}$ is closable if and only if the operator $\mathcal{R}_{1}(s)+\mathcal{R}_{2}(s)$ is closable for $\nu$-a.e. $s$, in which case $s \rightarrow \overline{\mathcal{R}_{1}(s)+\mathcal{R}_{2}(s)}$ is a $\nu$-measurable family of operators from $\mathfrak{S}_{1}$ to $\mathfrak{S}_{2}$ and $\overline{R_{1}+R_{2}}=\int \oplus \overline{\mathcal{R}_{1}(s)+\mathcal{R}_{2}(s)} d \nu(s)$.

Proof. Let $\mathfrak{H}_{1,2}=\int^{\oplus} \mathfrak{S}_{1,2}(s) d \nu(s)$ and $R=\int^{\oplus}\left(\mathcal{R}_{1}(s)+\mathcal{R}_{2}(s)\right) d \nu(s)$. Clearly, $R$ is an extension of $R_{1}+R_{2}$. Let $(\xi, \eta) \in G_{R}$. Then $\xi(s) \in D_{\mathcal{R}_{1}(s)} \cap D_{\mathcal{R}_{2}(s)}$ for $\nu$-a.e. $s$. Let $\eta^{(1)}$ and $\eta^{(2)}$ be $\nu$-a.e. defined sections of $\mathfrak{S}_{2}$ such that $\eta^{(1,2)}(s)=\mathcal{R}_{1,2}(s) \xi(s)$ for $\nu$-a.e. $s$. By Lemma B.10 $\eta^{(1)}$ and $\eta^{(2)}$ are $\nu$-measurable. Let $A_{1} \subset A_{2} \subset \ldots$ be a sequence of elements of $D_{\nu}$ such that $S_{\nu}=\bigcup_{k=1}^{\infty} A_{k}$ and $\left\|\eta^{(1)}(s)\right\|+\left\|\eta^{(2)}(s)\right\| \leq k$ for $\nu$-a.e. $s \in A_{k}$. Set $\xi_{k}=\xi^{A_{k}}, \eta_{k}=\eta^{A_{k}}, \eta_{k}^{(1,2)}=\left(\eta^{(1,2)}\right)^{A_{k}}$. We obviously have $\xi_{k} \in \mathfrak{H}_{1}$ and $\eta_{k}, \eta_{k}^{(1,2)} \in \mathfrak{H}_{2}$ for all $k$. As $\left(\xi_{k}, \eta_{k}^{(1,2)}\right) \in G_{R_{1,2}}$ and $\eta_{k}=\eta_{k}^{(1)}+\eta_{k}^{(2)}$, we have $\left(\xi_{k}, \eta_{k}\right) \in G_{R_{1}+R_{2}}$ for all $k$. Since $\left(\xi_{k}, \eta_{k}\right) \rightarrow(\xi, \eta)$ in $\mathfrak{H}_{1} \oplus \mathfrak{H}_{2}$ as $k \rightarrow \infty$, we conclude that $G_{R_{1}+R_{2}}$ is dense in $G_{R}$ and, hence, $D_{R_{1}+R_{2}}$ is dense in $D_{R}$. In view of statement 2 of Proposition B.23, it follows that $R_{1}+R_{2}$ is densely defined if and only if $\mathcal{R}_{1}(s)+\mathcal{R}_{2}(s)$ is densely defined for $\nu$-a.e. $s$. Since $\bar{G}_{R}=\bar{G}_{R_{1}+R_{2}}$, the operator $R_{1}+R_{2}$ is closable if and only if $R$ is closable. In view of Lemma B.20, the latter condition holds if and only if $\mathcal{R}_{1}(s)+\mathcal{R}_{2}(s)$ is closable for $\nu$-a.e. $s$ (note that $s \rightarrow R_{1}(s)+\mathcal{R}_{2}(s)$ is a $\nu$-measurable family of operators from $\mathfrak{S}_{1}$ to $\mathfrak{S}_{2}$ by Lemma B.9). If $R_{1}+R_{2}$ is closable, then Lemma B.20 implies that $\overline{R_{1}+R_{2}}=\bar{R}=\int \oplus \overline{\mathcal{R}_{1}(s)+\mathcal{R}_{2}(s)} d \nu(s)$.

Remark B.27. Propositions B.21, B.22, and B.23 were proved in Ref. 7 (see also Ref. 47). They are generalizations of corresponding statements proved in Ref. 6 for bounded operators. Propositions B.25 and B.26 were obtained in Ref. 48 under additional assumption that $\nu$ is a finite Borel measure on a metrizable compact space and were proved in Ref. 43 in their general form.

${ }^{1}$ S. Albeverio, F. Gesztesy, R. Høegh-Krohn, and H. Holden, Solvable models in quantum mechanics, Texts and Monographs in Physics (Springer-Verlag, New York, 1988).

${ }^{2}$ D. M. Gitman, I. V. Tyutin, and B. L. Voronov, Self-adjoint extensions in quantum mechanics; general theory and applications to Schrödinger and Dirac equations with singular potentials, Progress in Mathematical Physics, Vol. 62 (Birkhäuser/Springer, New York, 2012).

${ }^{3}$ N. I. Akhiezer and I. M. Glazman, Theory of linear operators in Hilbert space. Vol. I, Monographs and Studies in Mathematics, Vol. 9 (Pitman (Advanced Publishing Program), Boston, Mass.-London, 1981) translated from the third Russian edition by E. R. Dawson, Translation edited by W. N. Everitt.

${ }^{4}$ A. G. Smirnov, "Generators of von Neumann algebras associated with spectral measures," J. Math. Anal. Appl. 398, 501-507 (2013).

${ }^{5}$ The counting measure on $\mathscr{S}$ is, by definition, the measure assigning to every finite subset of $\mathscr{S}$ the number of points in this set.

${ }^{6}$ J. von Neumann, "On rings of operators. Reduction theory," Ann. of Math. (2) 50, 401-485 (1949).

${ }^{7}$ A. E. Nussbaum, "Reduction theory for unbounded closed operators in Hilbert space," Duke Math. J. 31, 33-44 (1964).

${ }^{8}$ R. Pallu de la Barrière, "Décomposition des opérateurs non bornés dans les sommes continues d'espaces de Hilbert," C. R. Acad. Sci. Paris 232, 2071-2073 (1951).

${ }^{9}$ Throughout the paper, a.e. means either 'almost every' or 'almost everywhere'.

${ }^{10}$ Y. Aharonov and D. Bohm, "Significance of electromagnetic potentials in the quantum theory," Phys. Rev. (2) 115, 485-491 (1959).

${ }^{11}$ I. V. Tyutin, "Electron scattering on a solenoid," arXiv:0801.2167 [quant-ph] (translated from preprint FIAN 27, P.N. Lebedev Physical Institute, Moscow, 1974).

${ }^{12}$ R. Adami and A. Teta, "On the Aharonov-Bohm Hamiltonian," Lett. Math. Phys. 43, 43-53 (1998).

${ }^{13}$ L. Dąbrowski and P. Šťovíček, "Aharonov-Bohm effect with $\delta$-type interaction," J. Math. Phys. 39, 47-62 (1998).

${ }^{14}$ We endow $S$ with the topology induced from $\mathbb{R}^{2}$. The Borel $\sigma$-algebra of $S$ consists of all Borel subsets of $\mathbb{R}^{2}$ that are contained in $S$. The Borel structure on $A^{\phi}$ is induced from $S$.

${ }^{15}$ For brevity, we let $\mathcal{W}_{\theta}^{\phi}(s, E \mid x)$ denote the value of the function $\mathcal{W}_{\theta}^{\phi}(s, E)$ at the point $x: \mathcal{W}_{\theta}^{\phi}(s, E \mid x)=\left(\mathcal{W}_{\theta}^{\phi}(s, E)\right)(x)$ A similar notation will be used for any maps whose values are also maps.

${ }^{16}$ To compute the limit of $u_{\vartheta}^{\kappa}(E \mid r)$ as $\kappa \rightarrow 0$, one has to apply L'Hôpital's rule and use the equality $\Gamma^{\prime}(1+n) / \Gamma(1+n)=c_{n}-\gamma$ (see Ref. 49, Sec. 1.7.1, formula (9)).

${ }^{17}$ See Ref. 49, Sec. 7.2.4, formula (33).

${ }^{18}$ A. G. Smirnov, "Eigenfunction expansions for the Schrödinger equation with inverse-square potential," Theor. Math. Phys. (to be published); e-print arXiv:1508.07747 [math-ph].

${ }^{19}$ J. Dixmier, Les algèbres d'opérateurs dans l'espace hilbertien (algèbres de von Neumann) (Gauthier-Villars Éditeur, Paris, 1969) deuxième édition, revue et augmentée, Cahiers Scientifiques, Fasc. XXV.

${ }^{20}$ Here and subsequently, $S_{\nu}$ denotes the largest $\nu$-measurable set, see Sec. A2

${ }^{21}$ See Sec. A3

${ }^{22}$ Recall that a closed densely defined linear operator $T$ in a Hilbert space is called normal if the operators $T T^{*}$ and $T^{*} T$ have the same domain of definition and coincide thereon. In particular, self-adjoint and unitary operators are normal.

${ }^{23}$ B. Fuglede, "A commutativity theorem for normal operators," Proc. Nat. Acad. Sci. U. S. A. 36, 35-40 (1950).

${ }^{24}$ M. A. Naümark, Linear differential operators. Part II: Linear differential operators in Hilbert space, With additional material by the author, and a supplement by V. È. Ljance. Translated from the Russian by E. R. Dawson. English translation edited by W. N. Everitt (Frederick Ungar Publishing Co., New York, 1968). 
${ }^{25}$ G. Teschl, Mathematical methods in quantum mechanics, with applications to Schrödinger operators, Graduate Studies in Mathematics, Vol. 99 (American Mathematical Society, Providence, RI, 2009).

${ }^{26}$ J. Weidmann, Spectral theory of ordinary differential operators, Lecture Notes in Mathematics, Vol. 1258 (Springer-Verlag, Berlin, 1987).

${ }^{27}$ Throughout this section and Sec. VII all equivalence classes are taken with respect to $\lambda_{a, b}$. We shall drop the subscript and write $[f]$ instead of $[f]_{\lambda_{a, b}}$.

${ }^{28}$ F. Gesztesy and M. Zinchenko, "On spectral theory for Schrödinger operators with strongly singular potentials," Math. Nachr. 279, 1041-1082 (2006).

${ }^{29}$ D. M. Gitman, I. V. Tyutin, and B. L. Voronov, "Self-adjoint extensions and spectral analysis in the Calogero problem," J. Phys. A 43, 145205, 34 (2010).

${ }^{30}$ A. Kostenko, A. Sakhnovich, and G. Teschl, "Weyl-Titchmarsh theory for Schrödinger operators with strongly singular potentials," Int. Math. Res. Not. 2012, 1699-1747 (2012)

${ }^{31}$ As in Sec. VI] we write $[f]$ in place of $[f]_{\lambda_{a, b}}$, where $\lambda_{a, b}$ is the restriction to $(a, b)$ of the Lebesgue measure $\lambda$ on $\mathbb{R}$.

${ }^{32}$ We denote by $\xi^{\prime}(s \mid r)$ the derivative of $\xi(s)$ at point $r: \xi^{\prime}(s \mid r)=(\xi(s))^{\prime}(r)$.

${ }^{33}$ Given $\psi=\left(\psi_{1}, \psi_{2}\right) \in \mathfrak{h} \oplus \mathfrak{h}$, we set $\bar{\psi}=\left(\bar{\psi}_{1}, \bar{\psi}_{2}\right)$, where $\bar{\psi}_{1}$ and $\bar{\psi}_{2}$ are elements of $\mathfrak{h}$ such that $\bar{\psi}_{1,2}(r)=\overline{\psi_{1,2}(r)}$ for $\lambda$-a.e. $r \in(a, b)$.

${ }^{34} \mathrm{H}$. Bauer, Measure and integration theory, de Gruyter Studies in Mathematics, Vol. 26 (Walter de Gruyter \& Co., Berlin, 2001) translated from the German by Robert B. Burckel.

${ }^{35} \mathrm{It}$ is easy to see that the intersection of any set of $\alpha$-classes is again an $\alpha$-class (note that such an intersection is always nonempty because $\varnothing$ is an element of every $\alpha$-class). Hence, given a set of sets $\mathcal{K}$, there exists the smallest $\alpha$-class containing $\mathcal{K}$.

${ }^{36}$ D. L. Cohn, Measure theory (Birkhäuser, Boston, Mass., 1980).

${ }^{37}$ G. W. Mackey, "Borel structure in groups and their duals," Trans. Amer. Math. Soc. 85, 134-165 (1957).

${ }^{38}$ V. A. Rohlin, "On the fundamental ideas of measure theory," Mat. Sbornik N.S. 25(67), 107-150 (1949).

${ }^{39}$ Note that a Hilbert space is separable if it is either finite-dimensional or infinite-dimensional with a countable orthonormal basis. In particular, we consider $\mathbb{C}$ as a one-dimensional Hilbert space with the scalar product $\langle\alpha, \beta\rangle=\bar{\alpha} \beta, \alpha, \beta \in \mathbb{C}$.

${ }^{40}$ M. S. Birman and M. Z. Solomjak, Spectral theory of selfadjoint operators in Hilbert space, Mathematics and its Applications (Soviet Series) (D. Reidel Publishing Co., Dordrecht, 1987) translated from the 1980 Russian original by S. Khrushchëv and V. Peller.

${ }^{41} \mathrm{~A}$ detailed account of the reduction theory for bounded operators can be found in Ref. 19 .

${ }^{42}$ M. H. Stone, "On unbounded operators in Hilbert space," J. Indian Math. Soc. (N.S.) 15, 155-192 (1951).

${ }^{43}$ M. J. J. Lennon, "On sums and products of unbounded operators in Hilbert space," Trans. Amer. Math. Soc. 198, 273-285 (1974).

${ }^{44}$ Indeed, let $\mathfrak{H}_{1}$ and $\mathfrak{H}_{2}$ be Hilbert spaces, $R$ be a closed operator from $\mathfrak{H}_{1}$ to $\mathfrak{H}_{2}, P$ be the orthogonal projection of $\mathfrak{H}_{1} \oplus \mathfrak{H}_{2}$ onto $G_{R}$, and $\psi_{1}, \psi_{2}, \ldots$ be elements of $\mathfrak{H}_{1}$ whose linear span is dense in $\mathfrak{H}_{1}$. If $\left(\psi, \psi^{\prime}\right) \in G_{R}$ is orthogonal to all vectors $P\left(\psi_{j}, 0\right)$, then $\left\langle\psi, \psi_{j}\right\rangle=\left\langle\left(\psi, \psi^{\prime}\right), P\left(\psi_{j}, 0\right)\right\rangle=0$ for all $j$ and, hence, $\psi=0$. As $G_{R}$ is a graph of an operator, it follows that $\psi^{\prime}=0$. Thus, the linear span of $P\left(\psi_{j}, 0\right)$ is dense in $G_{R}$.

${ }^{45} \mathrm{M}$. Reed and B. Simon, Methods of modern mathematical physics. IV. Analysis of operators (Academic Press [Harcourt Brace Jovanovich Publishers], New York, 1978).

${ }^{46}$ F. Gesztesy, A. Gomilko, F. Sukochev, and Y. Tomilov, "On a question of A. E. Nussbaum on measurability of families of closed linear operators in a Hilbert space," Israel J. Math. 188, 195-219 (2012).

${ }^{47}$ C. Lance, "Direct integrals of left Hilbert algebras," Math. Ann. 216, 11-28 (1975).

${ }^{48}$ P. G. Dixon, "Unbounded operator algebras," Proc. London Math. Soc. (3) 23, 53-69 (1971).

${ }^{49}$ A. Erdélyi, W. Magnus, F. Oberhettinger, and F. G. Tricomi, Higher transcendental functions. Vols. I, II (McGraw-Hill Book Company, Inc., New York-Toronto-London, 1953) based, in part, on notes left by Harry Bateman. 